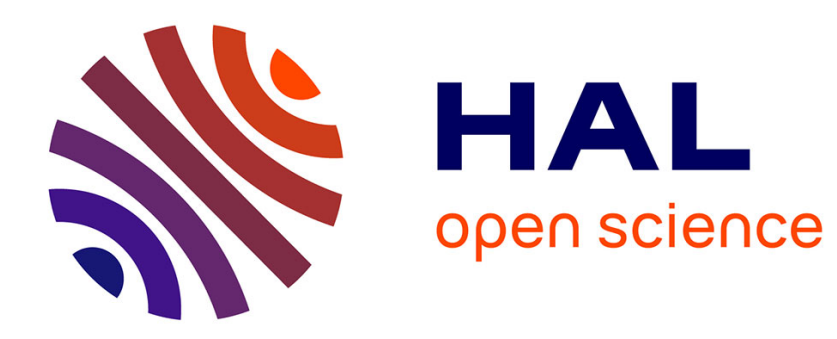

\title{
Les lois du silence. Essai sur la fonction sociale du secret Jean Jamin
}

\section{To cite this version:}

Jean Jamin. Les lois du silence. Essai sur la fonction sociale du secret. François Maspéro, 134 p., 1977, Dossiers africains (ISSN 0335-8062), Marc Augé et Jean Copans. halshs-00376244

\section{HAL Id: halshs-00376244 https://shs.hal.science/halshs-00376244}

Submitted on 17 Apr 2009

HAL is a multi-disciplinary open access archive for the deposit and dissemination of scientific research documents, whether they are published or not. The documents may come from teaching and research institutions in France or abroad, or from public or private research centers.
L'archive ouverte pluridisciplinaire HAL, est destinée au dépôt et à la diffusion de documents scientifiques de niveau recherche, publiés ou non, émanant des établissements d'enseignement et de recherche français ou étrangers, des laboratoires publics ou privés. 
dossiers africains

\section{Jean Jamin}

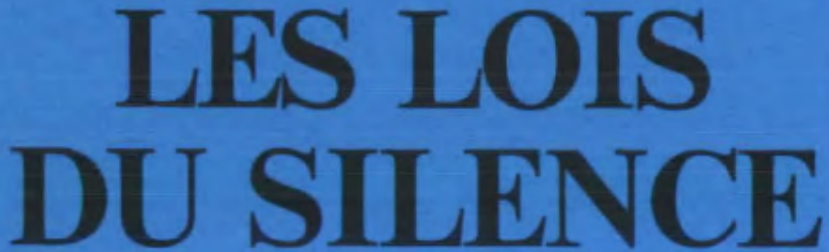

ESSAI SUR LA FONCTION SOCIALE DU SECRET

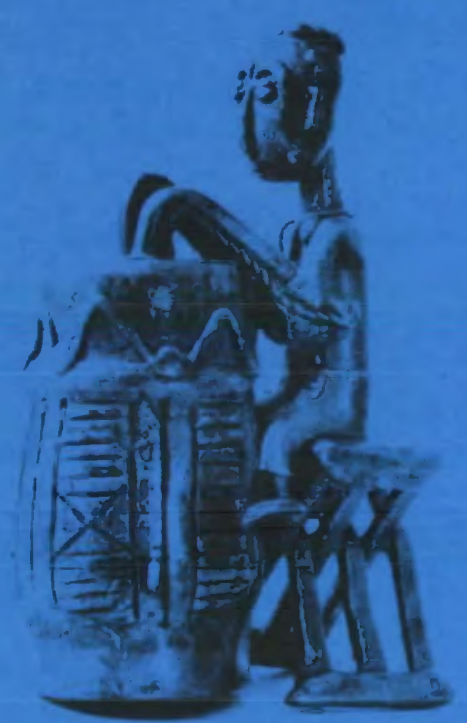


Tous nos chaleureux remerciements à François Gèze, directeur des éditions La Découverte, et propriétaire du Fonds Maspèro, pour son autorisation de mettre en ligne cet ouvrage en archives ouvertes (maquette propriétaire).

Jean Jamin

Eliane Daphy (responsable des archives ouvertes du IIAC)

\section{Pour citer cet ouvrage en archives ouvertes :}

Jamin Jean, 2009, Les lois du silence. Essai sur la fonction sociale du secret, OAI halshs-00376244 http://halshs.archives-ouvertes.fr/halshs00376244/fr/ (facsim. num. : 1977, Paris, François Maspéro, 134 p.)

ISBN : 2-7071-0920-7

ISSS : 0335-8062

Notice Sudoc : 000585920

Références BNF : FRBNF34703681

oai:halshs.archives-ouvertes.fr:halshs-00376244_v1

Jean Jamin

LAHIC-IIAC UMR8177 EHESS

http://www.lahic.cnrs.fr/article.php3?id article $=88$

jamin(at)ehess.fr 


\title{
ECOLE DES HAUTES ETUDES \\ EN SCIENCES SOCIALES \\ Centre d'études africaines
}

\section{DOSSIERS AFRICAINS}

\author{
dirigés par \\ Marc Augé et Jean Copans
}


(C) François Maspero, 1977

ISBN 2-7071-0920-7 


\title{
JEAN JAMIN
}

\author{
LES LOIS \\ DU SILENCE
}

ESSAI

SUR

LA FONCTION SOCIALE

DU SECRET

\section{FRANÇOIS MASPERO}

1, place Paul-Painlevé, V

Paris

1977 
A J. of J.G. Rozoy 
Dommage qu'il n'y ait rien de merveil leux dans les signes, ni de significatif dans les merveilles! Il y a une clef quelque part... attendez... chut, silence!

Hermann Melville, Moby Dick.

En vérité, cet officier semblait avoir la mission spéciale de protéger la dignité souveraine du capitaine qui, en quelque sorte, paraissait être trop plein de dignité dans sa personne pour condescendre d la proseger lui-même.

Hermann Melville, Redburn. 


\section{INTRODUCTION}

La plupart des recherches portant sur les traditions orales accordent bien sûr, par définition et par vocation, un statut prioritaire, pour ainsi dire dominant, à la parole : voie par laquelle se transmet le savoir et se reproduisent les sociétés lignagères. Celles-ci, on l'a dit, ont une civilisation de l'oralité, possèdent une littérature orale. L'ambiguïté de ces concepts traduit assez bien l'embarras des observateurs devant une réalité sociale et culturelle - tantôt définie négativement (sans écriture), tantôt d'une manière contradictoire (à littérature orale), tantôt avec un certain fixisme (à tradition orale) - tout entière perçue et contenue, mais en quelque sorte en creux, par l'absence et le manque (ceux de l'écriture), dans le champ du discours et de la parole. On ne peut certes contester aussi rapidement des analyses et des études dont la finesse et la qualité ne sont plus à vanter : il s'agit plutôt d'en relativiser la portée et d'ouvrir d'autres perspectives en s'interrogeant notamment sur les conditions sociales d'exercice de la parole; conditions qui doivent, au bout du compte, en infléchir le sens et la valeur et permettre de dégager le mode d'articulation entre les structures de codification, de communication et de subordination. Si la parole est prise, elle se trouve également prise dans un réseau social qui en conditionne la pertinence et la fréquence, qui en limite donc l'utilisation. N'importe qui ne dit certes pas n'importe quoi, n'importe quand et n'importe où. Telle peut être, résumée d'une 
façon lapidaire, l'hypothèse de départ, qui amènera à poser des questions du type : qui dit quoi ? quoi dit quoi ? quoi dit qui ?

Il faut tout d'abord se démarquer des perspectives et analyses linguistiques et sémiologiques, car c'est moins l'étude de la langue, des discours proprement dits, des codes, des signes ou du symbolique qui nous intéresse ici que celle de leur fonctionnement, de leurs usages, mésusages ou non-usages par des acteurs sociaux en situation et en relation. Toute parole, tout discours, qu'il soit tenu ou retenu, met en place et en scène des groupes ou des catégories sociales qui sont dans un rapport aux pouvoir-dire et aux savoir-dire, qui définissent selon une logique à découvrir des pouvoir-faire et des savoir-faire.

Les commentaires et réflexions sur les conditions d'enquête et d'observation, qui souvent ouvrent ou jalonnent les monographies, soulignent cette socialisation de la parole et cette position du discours. Il ne suffit plùs de recueillir et d'enregistrer l'information, la parole donnée. Il convient de la situer, de la confronter, d'en faire l' $*$ histoire », la 《 géographie » et la 《 généalogie », d'autant qu'obtenue souvent par interrogation - elle-même perçue par les enquêtés comme menaçante ou au mieux inconvenante - elle risque sans cela d'être complaisante, conventionnelle, trompeuse ou insipide. Cette prudence initiale, presque devenue une mode ou une clause de style, élude toutefois le problème qu'elle dévoile, par son côté fréquem. ment descriptif, historique et quelquefois idiosyncrasique. Cependant, les travaux de quelques psychologues et psychothérapeutes africanistes, notamment ceux de l'équipe de Fann à Dakar ${ }^{1}$, confrontés dans leur pratique aux situations d'écoute, de dialogue et de discours, cernent de plus près, semble-t-il, cette question du statut de la parole ${ }^{2}$. Certains font ressortir ce qui, jusqu'alors, pouvait sembler paradoxal pour une société traditionnelle, à savoir le danger, la menace, la violence et le viol des paroles ${ }^{3}$; d'autres insistent sur l'ambiguïté du dire, tour à tour régulateur et perturbateur, consolateur et accusateur, et mettent en évidence des processus éducatifs qui tendent justement à le pondérer, le temporiser, le retenir, le suspendre, qui apprennent en somme à savoir se taire $>$ ".

1. Cf. la revue Psychopatbologie africaine.

2. Cf. E. et M.C. ORTigues, A. et J. ZEMPLÉnI, Psychologie clinique et Ethnologie (Sénégal) », Bulletin de psychologie, XXI, 270, Paris, 1968, p. $950-958$.

3. Cf. D. Storper-Perez, La Folie colonisée, Maspero, Paris, 1974, p. 16-20.

4. J. Rabann-Zempléni, e Expression de l'agressivité... », art. cité, 1974, 
D'une façon indépendante, à un niveau plus sociologique, $M$. Augé fait des remarques analogues lorsqu'il écrit que, dans la société lignagère, \& la théorie enseigne avant tout à se taire, elle révèle les dangers de la prise de parole, elle menace de condamner ceux qui auraient l'imprudence de recourir à elle pour élaborer un discours effectivement dit, une accusation effectivement formulée ${ }^{5} \gg$. Condamnée à la parole, la société lignagère apprendrait-elle à s'en méfier, à s'en garder? La loi sociale serait-elle ici, comme le suggère par ailleurs M. Augé, une loi du silence où la stratégie du pouvoir consisterait précisément à taire et à se taire ${ }^{6}$ ? De ce point de vue, le régime des secrets qui entoure certaines pratiques rituelles clés, telle l'initiation, pourrait être l'expression privilégiée de cette loi, et l'on peut s'interroger sur leurs fonctions sociales.

De tels processus ne sont peut-être pas propres aux sociétés traditionnelles ou lignagères. S'ils apparaissent ici grossis et amplifiés, par conséquent plus visibles et accentués, sans doute plus pertinents et particuliers, du fait de la taille et de la structure de ces sociétés, on peut néanmoins supposer qu'ils jouent et se révèlent quelque part dans les sociétés dites avancées. Ce qui, au bout du compte, à condition que l'hypothèse soit vérifiée, permettrait de s'interroger sur la structure du pouvoir, sur les conditions théoriques de son exercice et, d'une façon plus générale, sur les stratégies de la communication sociale?

La dimension et l'organisation des sociétés \& avancées $\gg$ tendent certes à décentrer et à démultiplier les lieux et les niveaux d'exercice du pouvoir, à élargir et à diversifier les réseaux de communication, à structurer et à institutionnaliser les rapports aux savoirs; en somme à diversifier les points d'ancrage et les modes d'expression du pouvoir. Mais les appareils et dispositifs mis en place et en cuvre obéissent peut-être à cette loi organique, repérable à des niveaux élémentaires, fondée sur le silence et la rétention. C'est en tout cas ce que l'on peut inférer des analyses de $\mathrm{M}$. Crozier sur le phénomène bureaucratique $^{8}$ : pour lui, le pouvoir naît de situations d'incertitude, de flou et de silence. Chaque groupe tend à augmenter la part d'incerti-

et L'Enfant wolof de deux d einq ans, op. cit., 1975, p. 409 et s. [les mentions op. cit et a art. cité \ renvoient à la bibliographie p. 128-131].

5. M. AUGE, Thborie des pouvoirs et idbologia..., op. cit., 1975, p. 226.

6. M. AUGE, La Construction du monde..., op. cit., 1974.

7. Cf. P. ROQuepio, Le Partage du savoir..., op. cit., 1974.

8. Lo Pbenomdne bureasucratique, Le Seuil, Paris, 1963. 
tude qu'il fait planer sur les autres et, par là même, son pouvoir à réduire l'incertitude déployée par les autres et donc à réduire leur pouvoir ${ }^{9}$. La centralisation généralement observée dans ce type d'organisation * procède moins d'une tentative de concentrer un pouvoir absolu au sommet de la pyramide » que d'une volonté « de placer une distance ou un écran protecteur suffisant entre ceux qui ont le droit de prendre une décision et ceux qui seront affectés par cette déci$\operatorname{sion}^{10} \gg$.

Sur un plan global et d'une façon assez spectaculaire, les événements de Mai 1968 ont été pour certains ${ }^{11}$ caractérisés par la \& prise de parole \$, par le rejet du silence quotidien, oppresseur, par celui des secrets de la décision : il s'agissait de bouleverser les «ègles politiques » de la communication, de la «massifier », de la démocratiser... Le cas français qui sera analysé au début et qui orientera notre problématique n’a ni cette exemplarité ni cette résonance; mais il a l'avantage, tout en étant plus sourd, plus dissimulé, de révéler des mécanismes de rétention liés à des exercices de pouvoir ordinaires et quotidiens. L'associer au domaine africaniste ne procède donc pas d'une intention comparatiste, mais répond plutôt à des exigences structurales. Il s'agit, comme nous l'avons dir plus haut, de cerner les conditions sociales d'exercice de la parole et, d'une façon plus générale, celles de la communication à partir de son négatif, le secret : le nondire plutôt que le non-dit. Cette démarche permet de repérer d'emblée des règles précises et visibles, quasi institutionnelles, de communication et de rétention. Elle peut, au bout du compte, permettre de définir un cadre et un protocole d'analyse pour saisir le principe d'articulation entre les structures de communication et de subordination.

On s'interrogera moins sur le contenu du secret que sur son mode de constitution et d'implication, sa forme et sa fonction. Il est toutefois certain, nous le verrons pour les tendeurs ardennais, que le contenu a son importance et qu'on ne définit pas «n'importe quoi » comme étant secret; mais il existe également - ainsi en Afrique, lors de certaines initiations - des « secrets de polichinelle \$ qui sont moins objets de connaissance et d'apprentissage que signes de reconnaissance et d'appartenance sociales, qui ont pour effet de partager socialement et géographiquement les discours et les savoirs. Ce qui importe dans

9. Cf. aussi L. SFEz, Critique de la décision, op. cit., 1973, p. 288.

10. M. CrozIER, La Société bloquée, Le Seuil, Paris, p. 95. 1968.

11. Cf. M. DB CERTEAU, La Prise de parole, Desclée de Brouwer, Paris, 
ce cas n'est pas tant l'acquisition d'un savoir caché que l'opération de masquage, l'affirmation de sa possession, la décision sociale, voire politique, de son droit d'usage. La nécessité imposée à tout initié de se taire et de savoir se taire - outre le fait qu'elle maintient une solidarité organique très forte, sorte de consensus en négatif, et outre le fait que ce qui doit être tu par quelques-uns peut être connu de tous - fait socialement exister le secret et partage l'univers social de la communication en dévoilant et en imposant tout un système de droits d'expression et de devoirs de rétention. Le secret intervient là comme repère et argument hiérarchiques. Son importance réside moins dans ce qu'il cache que dans ce qu'il affirme : l'appartenance à une classe, à un statut.

Compte tenu de ces remarques préliminaires, je ferai un certain nombre de propositions et d'hypothèses :

- toute parole sociale peut et doit être interprétée en termes de pouvoir, qui est précisément et avant tout celui de dire ou de ne pas dire. Dans cette alternative, le choix et sa reconnaissance sociale définiraient l'ascendant et la place hiérarchique ;

- de ce point de vue, ce serait moins l'usage que la possession de la parole qui créerait l'ascendant (dans la mesure où tout ce qui est dit peut être contredit);

- chaque position sociale s'accompagne et se marque de non-dit et de non-dire qui tendent à l'affirmer et à la maintenir. Le halo des silences, le jeu des secrets, la rétention de la parole interviennent comme seuil, comme barrière et niveau. Cela peut amener à repenser la transmission du savoir suivant une perspective horizontale et non plus verticale;

- tout savoir-dire ne définit pas forcément un pouvoir-dire. La proposition inverse peut être également retenue;

- la connaissance des secrets suppose ou implique un savoir-taire qui définit un pouvoir-dire.

Les exemples illustrant ces propositions ont été principalement choisis en fonction d'un itinéraire professionnel et scientifique propre Ce qui explique, d'une part, leur dispersion géographique; ce qui accuse, d'autre part, le caractère d'essai donné à cet ouvrage. Il ne s'agit pas en effet de faire une théorie de la parole et de la communication, du silence et de la rétention. On se propose plutôt d'ouvrir quelques perspectives, de reproblématiser un domaine jusqu'alors peu interrogé et peu contesté, de jeter enfin quelques bases visant à élahorer une sociologie du non-dire et, pourquoi pas, du non-dit. 


\section{I \\ SECRET CYNÉGETIQUE \\ ET \\ POUVOIR COMMUNAL}

La tenderie aux grives est une technique de piégeage traditionnelle se pratiquant exclusivement dans les massifs forestiers du plateau ardennais. L'ancienneté des procédés, attestée par la fabrication artisanale du métier, traduit d'emblée son archaïsme, son particularisme, son caractère quasi résiduel et marginal. Dès lors, il peut paraître surprenant d'inaugurer notre propos par son étude, de lui consacrer une large part, de l'associer au domaine africaniste, de lui donner enfin valeur d'ouverture. Il y a plusieurs raisons à ce choix. En premier lieu, c'est au cours de cette recherche que sont apparus avec acuité, concrètement et à échelle réduite, les mécanismes et articulations des faits de confiscation et de rétention des savoirs. C'est ainsi que la mise en évidence et l'analyse des taxonomies dites populaires firent apparaître des variations classificatoires sociologiquement pertinentes qui remettaient en cause la démarche ethnoscientifique américaine, (notamment fondée sur le postulat implicite de « l'équivalence cognitive > des informateurs), et posèrent le problème de la distribution et de la transmission sociales des connaissances de même que celui, lié, de leur légitimation, accumulation et manipulation.

En second lieu, l'ampleur, la vivacité et la coloration politique des réactions locales devant les menaces d'interdiction répétées de la tenderie, proférées par les autorités administratives sous la pression des sociétés de protection de la nature et de quelques sociétés de 
chasse (pour une fois et à ce propos alliées), actualisaient et incorporaient socialement sa pratique, du moins au niveau communal (déplaçaient et contestaient donc sa marginalité d'abord perçue et volontiers affirmée dans les rapports administratifs). Ce phénomène posait alors la question du pouvoir social et idéologique de son personnel, au demeurant très réduit ${ }^{1}$, par là même remarquable quant à son influence locale. Ces situations conflictuelles accentuèrent et dévoilèrent, par cette mise en scène brutale, tout un jeu du secret et toute une stratégie de la dissimulation.

En troisième lieu, enfin, le choix du piégeage comme ouverture et pôle peut se justifier par une métaphore : il paraît être orchestration du silence et de l'absence. Son efficacité, son « pouvoir technique 》 supposent en effet la dissimulation, la retenue et la distance, à l'inverse de la chasse où la relation à l'animal est paradoxalement raccourcie, au moyen de l'arme et du projectile, à une distance minimale : le gibier doit être vu, repéré et approché pour que le coup ait toutes les chances de porter. L'efficacité requiert donc ici la présence individuelle ou collective de l'homme ${ }^{2}$. L'écart minimal techniquement, voire idéologiquement, affirmé entre l'homme et sa proie tend à singulariser, à individualiser, à focaliser le rapport ethnozoologique. Dans le piégeage, la distance est au contraire agrandie. L'écart est théoriquement maximal. L'efficacité technique du piège suppose l'absence individuelle ou collective de l'homme ${ }^{3}$. Cela est coextensif à un élargissement de la relation homme-animal, qui devient générique ou au mieux spécifique. Le piégeage excluant, sous peine de nuire à sa finalité, le contrôle physique, il convient d'y suppléer par le contrôle écologique du piège : il s'agit de l'adapter à une espèce déterminée. En conséquence - et à l'inverse de la chasse où l'emploi de l'arme, instrument écologiquement non spécialisé en tant que prolongement du bras, implique une agression directe, une distance minimale ex un

1. Une centaine de tendeurs pour l'ensemble du département. Ce nombre est quasiment constant depuis un siècle (profondeur donnée par les archives) et paraît socialement contrôlé; voir infra.

2. La chasse à l'affût est un cas particulier, d'ailleurs souvent méprisée par les vrais chasseurs.

3. Ce point a déjà été souligné par Lévi-Strauss dans son analyse de la chasse aux aigles chez les Hidatsa (cf. La Pensée sauvage, Plon, Paris, 1962, p. 68-72), et par M. Détienne dans celle des aromates en Grèce. (cf. Les Jardins d'Adonis, Gallimard, Paris, 1972, p. 39-47). Pour plus de détails et pour la critique de ces analyses, cf. J. JAMIN, La Tenderie aux grives..., op. cif., 1974, p. 8-15. 
rapport à l'individu —, l'utilisation du piège, dispositif écologiquement spécialisé, en tant que prolongement du milieu, définit une agression indirecte, une distance maximale et un rapport au genre. La situation ardennaise a ceci de remarquable qu'elle traduit sociologiquement cette opposition somme toute théorique, en différenciant, tant par la composition que par les origines, les deux groupes : tendeurspiégeurs/chasseurs, dont des conflits, parfois violents et ouverts, marquèrent leurs relations.

\section{Le piège de la parole}

Les grives se capturent à terre ou à la branche au moyen de collets faits en crin de cheval, placés dans des grivières (coupes affouagères), pendant les mois de septembre, octobre, novembre, période de migration des turdidés. Les conditions bioclimatiques du plateau ardennais, situé sur l'axe de migration des grives, font de cette région un piège écologique dont le piège humain ne serait que le prolongement : brouillards épais, crachin, ciel bas et couvert, températures fraîches, etc., autant de facteurs qui, en dissimulant les repères astronomiques et topographiques favorisant l'orientation des migrations, forcent les grives à séjourner dans la forêt où elles trouvent des succédanés de leur niche écologique.

Alors que le piège à terre, bayette, ne nécessite aucun appât - la malacofaune du sentier (base alimentaire des grives), soigneusement balayé de part et d'autre du piège, constituant la seule amorce -, le piège à l'arbre, pliette (sorte de perchoir au-dessus duquel se trouve le collet, lacs), est amorcé avec des baies de sorbier dont les grives sont friandes.

L'association et l'articulation de ces deux modes de capture dans un même temps et dans un même procès représentent une opération technique intéressante, révélatrice de certaines composantes socioculturelles et de certaines tensions sociales, puisqu'elles furent causes de conflits entre les chasseurs et les tendeurs.

Le rendement du piège à terre est supérieur à celui des pliettes : on estime généralement, pour deux fois moins de hayettes, que les captures se répartissent par moitié à l'arbre et à terre. Le soin et le temps mis (ici le double) à la fabrication et à la pose des pliettes 
ne sont donc pas du tout liés à des considérations techniques et/ou économiques de rendement, mais procèdent plutôt d'une idéologie gastronomique locale qui survalorise la grive prise à l'arbre, et qui justifie la mise en œuvre de procédés techniques et de démarches économiques sinon complexes, du moins relativement élaborés (outillage permanent : le fer à tendre, instrument servant à pratiquer des fentes dans la branche pour y fixer le perchoir-piège; circuits et modes d'acquisition et de conservation des baies de sorbier, etc.). En ce sens, le piégeage des grives à terre, en fait très peu valorisé sur le plan technique - il s'agit de planter un piquet au bout duquel on accroche le collet -, serait la déviation ou la transformation d'une action de piégeage dont l'objet aurait été autre. Certaines archives, notamment les Mémoires de F.S. Cazin, et certains entretiens avec des tendeurs permettent de le supposer. Cazin écrit en effet : « Le Culdessart à une lieue et demie de Rocroy est entouré des forêts du Prince de Chimay, de grands bois taillis où, après les vendanges, les grives viennent des vignes de la Champagne s'abattant par milliers. Les gens du pays établissent alors leurs tenderies; elles consistent en une baguette recourbée enfoncée par ses deux extrémités dans le corps d'une branche d'un buisson où passe également un nœud coulant en crin. A la partie inférieure de la baguette est appendu un petit bouquet de grains de branzière (sorbier). Chaque tendeur suit sa ligne, longeant, croisant d'autres tenderies, et jamais je n'ai entendu dire que des grives prises à l'une fussent dérobées par un tendeur voisin. Quelques-uns avaient jusqu'à dix mille lacets, nous en avions sept à huit cents. Dans les endroits marécageux, nous faisions avec des branches recourbées de petites hayes de quinze à vingt pieds de long ne laissant qu'un passage au milieu, à ce passage était un nœud coulant de quatre crins tordus et attachés à un piquet solidement enfoncé. Là se prenaient des bécasses".

Si donc des grives étaient capturées à terre, il fallait y voir un accident! Le piégeage à terre des grives pourrait donc être d'origine récente. Il serait apparu à la suite des conflits qui opposèrent les tendeurs aux chasseurs, ces derniers ayant finalement réussi à s'approprier le gibier à plume pour la capture duquel le piège à terre aurait été antérieurement conçu. Contraints par les événements, les tendeurs auraient redéfini la finalité des hayettes, en inventant au

4. F.-S. CAZIN, * Mémoires... *, art. cité, 1954, p. 6. C'est nous qui sou-
lignons. 
besoin une espèce de grive, la gratteuse (cf. infra), pour laquelle elles s'adaptaient, qui donc en justifiait la pose, mais qui camouflait d'autant mieux la finalité première, secrètement conservée. Si lon observe en effet l'importante concentration des hayettes dans les couverts très ajourés, peu fréquentés de ce fait par les grives, la nature du crin utilisé (de préférence le crin de mulet, plus solide) et les nœuds fabriqués ${ }^{s}$, il ressort que le but recherché (mais bien entendu non avoué) par quelques tendeurs, pour ne pas dire tous, est bien la capture de bécasses, gélinottes et faisans, celle des grives servant dans ce cas de couverture - alors qu'à l'arbre le piège apparaît tout à fait spécialisé pour leur capture. Cela peut, en outre, s'appréhender dans la destination post mortem des grives : celles prises à l'arbre rentrent préférentiellement dans les circuits de prestations jouant dans le réseau de parenté ou de pseudo-parenté dont nous tenterons de montrer plus loin qu'ils constituent la finalité « économique $\gg$ de la tenderie aux grives. Ainsi la distinction qui a été faite dans la présentation de ces deux modes de capture est-elle moins arbitraire. Leur articulation dans un même champ ceptologique est structurellement accidentelle. En somme, les deux dispositifs s'ajoutent plus qu'ils ne se complètent. Leur solidarité technique, pourtant opératoire dans la couverture écologique qu'elle implique, est conséquente à une situation historique qui a nécessité la redéfinition ceptologique de l'un (piège à terre) et son articulation écologique à l'autre (piège à l'arbre).

La juxtaposition de ces deux modes de capture dans un même procès, la coïncidence observée entre leur agencement dans un même lieu et le biotope de la grive, enfin le rendement supérieur de l'un (hayette) ne signifient donc pas qu'ils soient nécessairement, fonctionnellement et structurellement liés, car en effet comment comprendre, s'il en était ainsi, que le tendeur n'augmente pas les pièges à terre? Après tout, les Ardennais du plateau ont pu vouloir ne consommer que des grives consommant des baies de sorbier, comme ils ont pu vouloir ne prendre que des bécasses, gélinotres et faisans à terre !

5. Le règlement n'indiquant pas la façon des lacs, certains tendeurs rem. placent le noud coulant par un double nceud qui a l'avantage de ne pas se détendre et de pallier ainsi la rigidité du piquet techniquement inadapté à la capiure du gibier à plume, puisqu'elle lui permet de casser le collet par tractions répétées. 
Les tendeurs distinguent communément huit catégories de grives sur la base de critères de différenciation chromariques et écologiques, alors que les espèces connues et reconnues par le Centre de recherche sur les migrations des mammifères et des oiseaux du Muséum d'histoire naturelle de Paris, comme séjournant ou migrant dans les Ardennes, se limitent à quatre : la grive mauvis (Turdus iliacus), la grive musicienne ( $T$. philomelos), la grive litorne ( $T$. pilaris), enfin la grive draîne ( $T$. viscivorus). Cette anomalie s'explique, d'une part, par le fait que les tendeurs incluent les merles dans la catégorie grive et, d'autre part, par la constitution en espèce distincte de la femelle du merle à plastron, $T$. torquatus (voir fig. 1).

Ces huit catégories sont classées en deux ensembles distincts et opposés en fonction de critères de différenciation morpho-chromatiques : celles qui sont tachetées, celles qui ne sont pas tachetées (niveau $\mathrm{D}$ sur la fig. 1). Les \& consommateurs \$ rangent généralement les premières (tachetées) dans la catégorie grive; les secondes (non tachetées) dans la catégorie merle. Il ne semble pas que ces deux catégories

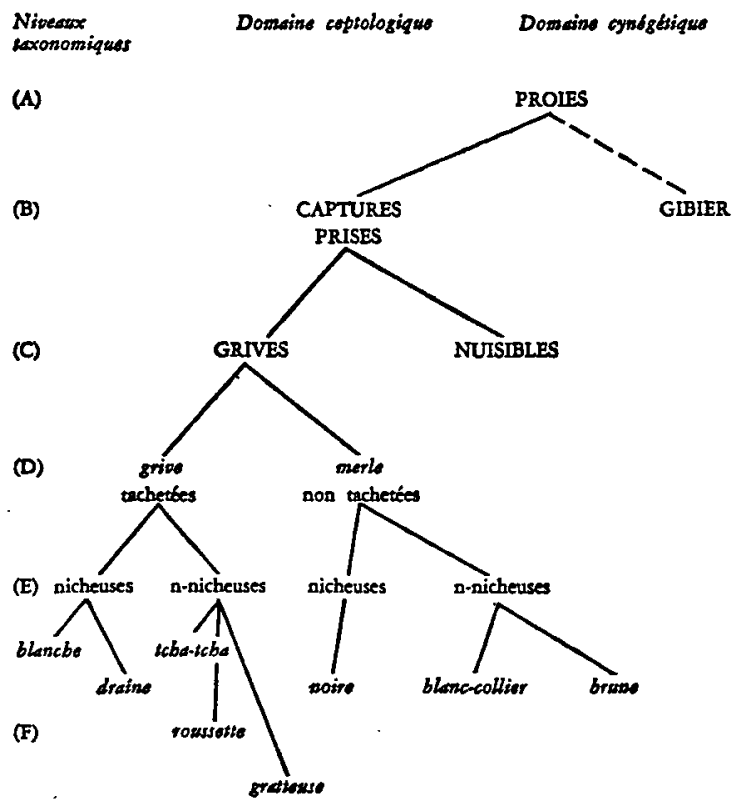

Fig. 1 : Syztime do clasification das tumdidfs. 
fonctionnent au niveau taxonomique des tendeurs : il s'agit dans leur cas d'ensembles organisés sur la base de caractères (attributs) opposés terme à terme, plutôt que d'ensembles terminologiquement distincts et fonctionnels au niveau lexical. Chacun de ces deux groupes est divisé en deux sous-ensembles définis par des critères de différenciation écologiques en relation d'opposition : celles qui nichent sur le plateau (nicheuses indigènes), celles qui ne nichent pas (non nicheuses migratrices).

La connaissance de cette classification, plus précisément la possession de ce savoir, varie bien sûr suivant l'implication technique des groupes sociaux dans le procès de production et s'altère par une catégorisation plus large et une disparition corrélative de l'attribution en fonction cette fois de la distance sociale des groupes au milieu. Uñe brève enquête (brève en raison de la relative simplicité de la taxonomie et du nombre restreint des groupes impliqués) portant sur sa distribution et son utilisation sociales confirme ces remarques. On peut dire dans un premier temps que le système classificatoire n'est pas transmis selon un processus éducatif « normal ». Les enfants des tendeurs, souvent employés comme « auxiliaires 》 dans certaines opérations techniques, ne semblent pas connaître les niveaux $E$ et $\mathbf{F}$ (cf. fig. 1), ou, s'ils les connaissent, paraissent les avoir oubliés; en tout cas ne les utilisent pas, n'en parlent pas, quels que soient par ailleurs le lieu et le moment social où s'inscrit la relation, comme si des mécanismes de « censure » ou d' « autocensure 》 entraient en jeu (encore qu'il puisse s'agir simplement et autrement d'une différence d'enjeu, de l'absence ou du peu de maîtrise du dispositif).

Le cas des femmes des tendeurs est plus probant : celles-ci, pourtant mieux impliquées techniquement (pose des pièges, relève des captures, en somme doublage des activités du tendeur; préparation et cuisson) et plus sollicitées socialement et économiquement (prestation, distribution et vente) que les enfants, ne paraissent pas distinguer les grives au niveau spécifique. La plus grande confusion est, en effet, observée lorsqu'il s'agit d'identifier les échantillons naturalisés présentés. Bien qu'elles connaissent, pour la plupart d'entre elles, les dénominations spécifiques, ces dernières ne semblent comporter aucun référent précis. Même en faisant varier la situation de l'enquête (présence ou absence du mari, entretiens répétés dans le temps), la confusion non pertinente (il ne s'agit pas d'une inversion dénotative ni d'une substitution de type métaphorique, mais plutôt d'une variation et d'une substitution de type métonymique) persiste. Ce qui signifie 
que, entre chacun des passages de l'enquêteur, le mari tendeur ne corrige pas et n'ajuste pas le niveau taxonomique utilisé par son épouse; ou bien, s'il le fait, la correction n'est pas retenue comme fonctionnelle par celle-ci. Le seul niveau opérant dans son cas reste l'opposition grive/merle, pertinente sur le plan culinaire : la chair de la grive est tendre et grasse, celle du merle est sèche et dure. A ce titre, la grive draîne et la grive litorne, fortement dévalorisées au niveau gastronomique, sont rangées dans la catégorie merle : « elles font partie des merles », disait-on en les désignant. Dans ce cas précis, il semble done que des critères de classification autres que chromatiques ou écologiques soient réellement utilisés : la taille et la qualité de la chair paraissent jouer dans le processus de différenciation des captures et de leur groupement en catégories distinctes selon un modèle qui ne coïncide pas avec celui défini par l'opposition tachetée/non tachetée, puisqu'il accepte l'inclusion dans une même classe d'éléments tachetés (draîne, litorne) et non tachetés (merle); ce qui permettrait de supposer un fonctionnement simultané des deux systèmes.

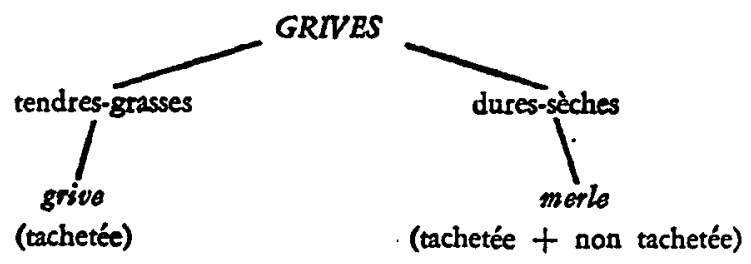

Fig. 2

L'implication technique ne définit donc pas nécessairement à elle seule la profondeur taxonomique : les femmes, les enfants qui interviennent dans la tenderie à des phases clés, notamment lors de la relève des captures où le contact à l'animal est direct, n'utilisent pas les niveaux $\mathrm{E}$ et $\mathrm{F}$. C'est donc moins la distance physique et technique que la distance sociale, soit le pouvoir de contrôler techniquement, économiquement et socialement les opérations, qui déterminerait dans notre cas les niveaux taxonomiques distingués et réellement utilisés. Ainsi, et du fait que la différenciation des grives en catégories spécifiques est socialement repérable au niveau des tendeurs adjudicataires, locataires ou propriétaires, c'est-à-dire des tendeurs contrô- 
lant l'ensemble des opérations, et que cette différenciation n'est pas verticalement communiquée, il est légitime de conjecturer une corrélation positive entre les niveaux taxonomiques utilisés et la position sociale des groupes ou des individus ". Au niveau local, la connaissance des grives semble donc à première vue confisquée par les tendeurs et distribuée sur un plan horizontal, c'est-à-dire à des individus dont le statut est reconnu comme réellement ou potentiellement identique. De ce point de vue, elle serait un signe d'appartenance et aurait moins une fonction technique qu'idéologique. Ce serait alors plus le savoir-dire (discours) que le savoir-faire (technique) qui, socialement et idéologiquement, définirait le tendeur.

Cependant, comme on a pu l'observer, le nombre d'éléments classés et les critères d'identification retenus ne sont ni très importants 'ni suffisamment complexes pour n'être pas facilement mémorisés, d'autant qu'ils ne sont pas réellement dissimulés par les tendeurs. Ia capacité diagnostique des femmes et des enfants, de même que leur maîtrise conceptuelle du dispositif expérimental ne sont certes ni contestées ni contestables, mais elles ne peuvent socialement s'exprimer. Dans cette perspective, ce serait moins la connaissance proprement dite qui serait appropriée par les tendeurs que son droit d'usage, c'est-à-dire le droit d'en parler, de l'utiliser, de la communiquer, de l'inculquer. La confiscation apparente du savoir, quasiment jonée par les partenaires sociaux de la tenderie, s'inscrirait en quelque sorte dans une stratégie de la reconnaissance et de l'affirmation sociales de la compétence, qui transformerait le savoir-dire en pouvoir-dire. Le renvoi à la position de tendeur pour décrire un ensemble, pour « nommer et organiser les choses », n'est pas aveu d'ignorance de la part des femmes ou des enfants, mais confirmation du pouvoir de légitimation et de sélection accroché au statut, maintenu, contrôlé, affirmé et constitué lors des séances d'adjudication des lots de tenderie

6. Ces remarques nous conduisent à poser la notion de taxonomie différentielle, qui peut être définie comme l'organisation hiérarchique de plusieurs ensembles d'éléments sur la base d'oppositions socialement engendrées, lexicalement fonctionnelles, variant suivant la problématique sociale des groupes qui les repèrent et les utilisent à des fins de communication et/ou de signalisation, et rangés en catégories de plus en plus vastes au fur et à mesure que s'agrandit la distance sociale aux éléments et que s'amenuise le pouvoir de les contrôler techniquement, économiquement et socialement. De ce fait, et en règle générale, la différenciation en catégories d'un ensemble donné doit varier en fonction de la signification socio-culturelle des éléments, et cela non seulement sur un plan horizontal (société globale-ensemble) mais aussi sur un plan vertical (groupes sociaux-ensemble). 
et par les secrets qui environnent la distribution des captures (cf. infra).

Ce pouvoir s'est notamment et historiquement tévélé d'une manière quasi exemplaire lors de \& l'affaire de la gratteuse \$. Celle-ci est la seule grive à être couramment décrite à partir de caractères éthologiques, et cela rompt d'une certaine façon la logique du système de classification. Elle passe après les mauvis, dont elle partage certains traits, vers la fin octobre, début novembre. Les tendeurs la décrivent comme plus petite : le sourcil est plus souligné et plus blanc, le roux du dessous des ailes est plus soutenu et va « jusqu'au cul \$. Elle a de chaque côté du bec un « poil \$ d'environ 1 centimètre qui Ia fait quelquefois nommer roussette à barbe. Sa capture ne s'opère qu'à terre et elle est rare. Elle est prise par la queue ou par les ailes, rarement par la tête. En se débattant, elle alerte les prédateurs et le tendeur ne trouve souvent que quelques plumes de part et d'autre de l'hayette. On la décrit ainsi : - C'est une petite grive, ressemblant étrangement à la grive mauvis qui précède. C'est le passage des gratteuses. Les baies ne les intéressent pas ; elles s'abattent, grattent dans les feuilles sur des ares et des ares de taillis car elles restent toujours en compagnie de cinquante à cent individus. Cette petite grive ne mange pas les baies de sorbier. Elle gratte sans arrêt toujours en reculant à la façon des poules, accumulant les feuilles mortes derrière elle en découvrant les petits vers. Puis elles partent au grand désespoir des tendeurs qui voient leurs lacs restés vides. Ces grives passent certainement toutes les années, mais seul le tendeur exercé peut s'en rendre compte. Or, bien souvent, le tendeur ne va plus au bois, ses lacs étant relevés au 15 novembre, et il confond la " gratteuse " et la " roussette " ?.

Le fait de "gratter à reculons, comme une poule \$, exprimé dans le terme qui la désigne, est donc retenu comme critère de différenciation d'avec les autres grives. Or, ce caractère est objectivement moins distinctif qu'il n'est affirmé par les tendeurs puisqu'il est partagé par l'ensemble des turdidés dans leur quête alimentaire. Des études précises, entreprises avec l'aide d'ornithologues, ne permirent pas d'identifier cette grive. 'Les descriptions faites par les tendeurs, seules informations disponibles étant donné qu'aucun d'entre eux n'avait conservé d'exemplaires sous une forme naturalisée ou congelée, renvoyaient toujours aux traits définitoires de la mauvis ou de

7. A. L., communication personnelle, décembre 1972. 
variétés intraspécifiques, accusés, soulignés, grossis et amplifiés. La gratteuse semblait être une mauvis surdifférenciée, mais pas du tout une espèce différente. Ces conclusions et cette dénomination particulière permirent de l'envisager comme relevant plutôt de l'ordre de la croyance que de celui de la connaissance ${ }^{8}$. Il s'agirait en somme d'une espèce inventée à des fins idéologiques... Il se pourrait en effet que son existence fût nécessaire à un moment de l'histoire de la tenderie. Le conflit de 1935, qui a opposé les chasseurs aux tendeurs, pourrait être la clé de compréhension et d'interprétation du phénomène du fait qu'il posa le problème de la tenderie à terre, donc du lieu où se capture spécialement la gratteuse.

Déjà, en 1904, Séjournet interprétait la décision d'interdire la tenderie, prise par le ministère de l'Agriculture en août 1903 à la suite de la loi du 30 juin 1903, comme la conséquence de pressions exercées par « les gros propriétaires de chasse \& qui « craignaient pour le gibier à plume (bécasse, gélinotte, faisans, etc.) soi-disant capturé à terre par les tendeurs ». Les interventions du député Dumaine et des sénateurs Gérard et Goutand permirent d'ajourner cette décision. L'annonce de la suppression de la tenderie à terre en Belgique par un arrêté royal du 25 octobre 1929 fir rebondir l'affaire et amorça le conflit de 1935. Déjà, à la suite des actions des conseillers généraux du département qui avaient pris en charge les intérêts des tendeurs, l'arrêté préfectoral de 1934 accordant aux tendeurs a le droit de préparer leur tenderie au mois de mars $\gg$, c'est-à-dire au moment où la « sève monte $\$$, avait suscité de vives protestations de la part des chasseurs pour qui cette mise en état ne pouvait être, à cette époque de l'année, que préjudiciable au gibier, donc à la chasse. Les démarches entreprises en août 1934 par L. Hubert, président d'une société de chasse du plateau, auprès du ministère de l'Agriculture, visant sinon la suppression de la tenderie à terre, du moins sa limitation et sa réglementation rigides, et la condamnation nette, en juin 1935, de cette pratique par le Conseil international de la chasse réuni à Bruxelles eurent pour principal effet de contraindre les autorités à réglementer la pose des lacs à terre, ainsi que cela fut précisé dans l'arrêté préfectoral du 23 août 1935 : \& Le lacet devra, sans qu'aucune branche ne puisse former ressort à déclenchement, être obligatoirement attaché à un piquet fixe

8. Cette impression me fut confirmée lorsque, à l'issue de plusieurs entretiens, les tendeurs me demandèrent : «ous y croyez, vous, à la gratteuse? 
et rigide d'au moins $0,30 \mathrm{~m}$ de longueur, ne dépassant pas du sol de plus de $0,20 \mathrm{~m}$, ayant au bout un diamètre minimum de $1 \mathrm{~cm}$, écorce comprise, et sous réserve que le brin inférieur du lacet ne se trouvera pas à plus de $6 \mathrm{~cm}$ au-dessus du sol: $\gg$

La fédération départementale veilla à l'application de ces mesures. Dans une lettre du 23 novembre 1935, L. Hubert, également président de la fédération de chasse des Ardennes, se plaignait du fait que des gardes avaient dû dresser trente-deux procès-verbaux pour la pose de lacs à terre non réglementaires, c'est-à-dire composés de cinq à six, voire huit crins. L'article premier de la réglementation précisait en effet que la tenderie aux grives et aux merles à la branche et -à terre ne pouvait se pratiquer qu'avec \& deux crins de cheval seulement, n'ayant pas plus de $30 \mathrm{~cm}$ de longueur $\gg-$ ces deux crins représentant théoriquement le seuil de résistance du collet aux tractions exercées par les grives capturées (le gibier à plume peut donc facilement les casser et se libérer en cas de prise accidentelle). C'était là une concession faite aux chasseurs. L. Hubert demandait en substance que des mesures énergiques soient prises afin de faire respecter les termes de l'arrêté et de punir sévèrement les tendeurs contrevenants. Ceux-ci réagirent en la personne de $\mathrm{P}$. Vienot, député socialiste de l'arrondissement de Mézières, qui prit leur défense et plaida leur cause auprès des autorités. Devant l'indécision administrative, la fédération de chasse, par l'intermédiaire de Hubert, porta les faits à la connaissance du ministre de l'Agriculture luimême, arguant des termes de l'article 4 de la loi du 30 juin 1903, et demanda la suppression pure et simple de la tenderie à terre. Le rapport de G.-M. Villenave du 4 décembre 1935 allait dans ce sens. La tenderie à la branche pouvait être maintenue à condition que le nombre de lacs posés en fût limité et contrôlé par une déclaration préalable en mairie, et sous réserve que le transport et la vente des grives fussent interdits en dehors du département des Ardennes ${ }^{\circ}$. Cela devait, concluait-il, amener progressivement la disparition de la tenderie aux grives. Suite à ce rapport, le ministre, par lettre du 21 janvier 1936, informait le préfet qu'il souhaitait voir la suppression de la tenderie à terre indiquée dans le prochain arrêté de réglementation. Dans sa réponse du 24 janvier, ce dernier notait que si « la suppression

9. Cette restriction avait des effets immédiats puisque l'acquisition des crins et des baies de sorbier, recherchés en dehors du département, se faisait souvent en échange de grives. 
était actuellement dévoilée, il y aurait des risques de protestations qui seraient défavorables à la veille des élections législatives $>$ et proposait en conséquence de la différer. Le Front populaire la laissa dans ses tiroirs...

Le conflit qui dura près de deux ans eut pour principal effet d'extérioriser la pratique de la tenderie aux grives et de mobiliser des forces politiques, tant pour sa suppression que pour sa défense, qui précisèrent les limites et les contours des groupes concernés et révélèrent les fondements sociaux de cette technique. Il s'était agi en fin de compte d'un conflit de classes dont le territoire - le \& contrôle des bois \(J. Lirry) - avait été l'enjeu visible. D'une part, \& les gros propriétaires de chasse \(en fait les adjudicataires) et leurs associés qui se recrutaient principalement parmi les industriels de la vallée de la Meuse; d'autre part, les petits paysans-ouvriers du plateau ardennais ${ }^{10}$ pour qui la tenderie était, en plus d'une amélioration du quotidien alimentaire, une manière de marquer leurs droits sur un territoire dont ils étaient collectivement propriétaires (forêts communales). L'argument invoqué par les chasseurs pour justifier leurs actions contre les tendeurs, argument selon lequel la tenderie à terre serait préjudiciable au gibier à plume, apparaît finalement comme un prétexte : au-delà de la tenderie à terre, c'était la tenderie aux grives qui était visée, comme d'ailleurs cela transparaît dans le rapport de Villenave, à l'élaboration duquel a participé la fédération de chasse par l'intermédiaire de ses gardes : « L'enquête prescrite par le ministère de l'Agriculture pour se documenter sur les conditions de cette tenderie en 1935 fut effectuée par les gardes de la fédération habilités à cet effet et donna lieu à l'établissement de trente-deux procès-verbaux. >

Le fait que des ouvriers parcouraient les bois que des industriels avaient loués aux communes ${ }^{12}$, non seulement les parcouraient mais encore les balisaient, inscrivaient leurs passages par le sentier et les pièges qui agissaient de la sorte comme marques, comme signes de possession et de contrôle d'un territoire, était socialement contradictoire (du fait de l'inversion de la domination) et parfaitement intolérable aux yeux des chasseurs qui voyaient ainsi une partie du contrôle cynégétique leur échapper. Ayant légalement la possibilité

10. Ardoisiers, cloutiers, boulonniers, bûcherons, etc.

11. Cettre situation tenait à l'adjudication séparée de la chasse et de la tenderie qui fut donc l'objet de locations distinctes à des attributaires différents. 
de se déplacer et d'opérer dans la forêt pendant près de cinq mois à des moments différents de l'année, le tendeur pouvait connaître l'importance, la composition, l'emplacement du gibier et intervenir sur ses déplacements ${ }^{12}$, donc limiter l'activité cynégétique par la maîtrise soit matérielle, soit cognitive de son objet ainsi que par la limitation de son territoire. Le savoir cynégétique des tendeurs, dont l'acquisition n'était pas contrôlée par les chasseurs, et qui de plus ne leur était pas communiqué, menaçait évidemment la position et la compétence de ces derniers du fait que la maîtrise conceptuelle des opérations leur échappait en se partageant. La parole retenue ébranlait le pouvoir détenu. Si le sentier du tendeur (voyette) est techniquement un passage, il devient sociologiquement une frontière qui entrave les évolutions des chasseurs moins comme obstacle naturel que comme obstacle social et finalement politique. C'est en ces termes qu'il convient d'interpréter cette déclaration de $\mathrm{L}$. Hubert dans sa lettre du 23 novembre 1935 : \& Il est absolument indispensable que les tendeurs aux grives comprennent que le gibier appartient aux chasseurs et non aux tendeurs. \$On ne peut être plus clair!

L'invention de la gratteuse eut pour fonction, au niveau de l'imaginaire, de décharger les accusations et de déjouer les interventions des chasseurs en justifiant du même coup la pratique de la tenderie à terre. Il s'agissait en somme de désamorcer la déviance ceptologique supputée (et souvent confirmée) et, en même temps, de la légitimer par l'affirmation d'une connaissance et d'une compétence dont le contenu et le fondement restaient, d'une manière paradoxale, secrètement conservés, selon la tactique du \& faire savoir que l'on sait - informer sans former, dire sans décrire - sans transmettre ni inculquer ce savoir. Cela limitait d'autant mieux les actions des pouvoirs publics. Un rapport anonyme de 1937 concluait en ces termes : * Bien qu'on ne l'ait jamais vue, il se pourrait, suivant les affirmations des tendeurs et la connaissance profonde qu'ils ont de la forêt, que cette grive existât. \ La question était en tout cas posée et devenait par là même aveu d'ignorance... à moins de devenir tendeur! Ainsi, en découvrant une espèce de grive dont la particularité, le trait distinctif, était sa capture à terre, dans le sentier du tendeur, les tendeurs rendaient nécessaire la pose des hayettes en les spécialisant. De ce fait, les prises de gibier ne pouvaient prêter à

12. En prélever même une partie : l'association tendeurs-braconnierscontrebandiers a souvent été invoquée. 
confusion : elles étaient forcément accidentelles et déculpabilisaient le tendeur. $\mathrm{La}$ constitution de la gratteuse fut donc historiquement nécessaire et son invention idéologiquement pertinente, puisqu'elle contrecarrait les tentatives d'interdiction de la tenderie à terre conséquentes aux accusations fondées sur sa finalité déviée. Le choix de l'association spécifique lui conférait en plus une valeur sociale et culinaire et en rendait sa capture indispensable, procédait donc de la demande locale, puisque les tendeurs la font ressembler à la roussette, c'est-à-dire à la grive la plus valorisée, dont elle est la queue de migration $\gg$.

\section{Le pouvoir silencieux}

Les tendeurs, à l'inverse des chasseurs, ne constituent pas un groupe aux contours sociaux définis et limités, dont les membres entretiennent entre eux des rapports de coopération technique ou économique (méthodes de chasse et répartition des rôles techniques, actions de chasse), partagent des règles et des rituels communs (réglementation interne, codification cynégétique, contingentement, répartition et consommation du gibier) et possèdent un lieu de réunion (\& cabane des chasseurs »). L'absence apparente et quasi totale de coopération, de relations et de communication entre tendeurs, la démarche technique individuelle, voire individualiste, font que ce \& groupe $\gg$ se repère uniquement par des relations externes, c'est-à-dire par les relations que ses membres établissent avec le milieu naturel et la société globale : « ceux qui parcourent et balisent les bois avec leurs pièges 》, « ceux qui distribuent et vendent les grives \$. Il s'appréhende lors des séances d'adjudication des lots de tenderie, principal mode d'acquisition des grivières.

Les locations de lots de tenderie par les communes sont sans doute de pratique très ancienne (pour Hercy, les archives permettent de les faire remonter jusqu'en 1870), et le produit en est quelquefois plus rémunérateur que celui des locations de chasse. L'adjudication se fait aux enchères publiques en mairie, une fois tous les cinq ans, par tirage au sort des lots à louer. La durée du bail de location est généralement d'une année renouvelable par tacite reconduction sur une période de cinq ans. La mise à prix des lots est fixée sur la base 
du loyer communal (soit entre 10 et $20 \mathrm{~F}$ actuels) et les enchères ne peuvent être inférieures à $10 \mathrm{~F}$. L'adjudicataire doit obligatoirement résider sur le territoire communal et retirer son permis de chasse à la mairie. Une telle clause vise certainement à protéger les tendeurs des ingérences extérieures, notamment de celles des chasseurs - dont la plupart étaient, par le passé, étrangers à la commune - qui pouvaient par voie d'adjudication accaparer une partie des lots et empêcher de la sorte la pratique de la tenderie. Cette interprétation paraît être confirmée par des mesures que nous avons relevées, différemment exprimées mais identiques quant au sens, dans les cahiers des charges qui ont été consultés : elles accordent à la commune le droit de reprise et de remise en adjudication de tout lot attribué mais non exploité : "Tout adjudicataire devra tendre obligatoirement son lot et ne pourra le céder en tout ou partie à une personne étrangère à la commune, sous peine de déchéance de ses droits et du paiement à titre de dommages et intérêts, au profit de la commune, d'une somme égale à une annuité de location. \Ces dispositions sont tout à fait remarquables et révélatrices de la puissance sociale des tendeurs. Elles indiquent clairement que la non-exploitation d'un lor ou sa cession à une personne étrangère à la commune sont vues comme \& lèse-commune », cette dernière s'arrogeant le droit de réclamer des dommages et intérêts et de déchoir le tendeur de ses droits d'exploitation. Elles garantissent et protègent la pratique de la tenderie en la rendant obligatoire une fois le lot attribué, et en la spécifiant sociologiquement ${ }^{13}$. De telles mesures visent donc moins à protéger les intérêts économiques de la commune pour laquelle et à ce niveau l'identité, la situation et la pratique de l'adjudicataire ne devraient pas jouer, le paiement annuel et anonyme des redevances suffisant à les garantir, qu’à préserver, localiser, voire monopoliser la pratique de la tenderie. Les cahiers des charges, et notamment les clauses que nous venons de mentionner, adoptés en séance plénière du conseil municipal, sont révélateurs du contrôle politique exercé par les tendeurs à l'échelle communale. Non seulement ils peuvent protéger leur activité, mais ils la réservent

13. Cela explique en partie que les quelques chasseurs de Hautey qui, à la suite de la communalisation des sociétés de chasse, avaient tenté d'accaparer et de confisquer des lots aux tendeurs lors des séances d'adjudication, afin de créer des * petites réserves pour le gibier $\gg$, c'est-à-dire d'en faire un no man's land soi-disant indispensable à la chasse, aient été obligés de tendre, soit d'entrer dans un système qu'ils se chargeaient de combattre, sous peine de perdre leurs droits. 
uniquement aux habitants de la commune, c'est-à-dire à des personnes dont l'insertion écologique et sociale est théoriquement identique. Cela se vérifie d'ailleurs au niveau idéologique, où seul le tendeur ardennais du plateau est considéré comme un vrai tendeur, et se maîtrise lors des séances d'adjudication où, exceptionnellement, les tendeurs se repèrent et se définissent comme groupe social et où, par le jeu des enchères, ils ont la possibilité d'accepter ou de rejeter l'éventuel postulant selon son degré de conformité social et idéologique. A cet égard, la lecture du montant des loyers, fixé après enchères et annuellement réglé par chaque tendeur, est un indicateur sociologique précieux. A Hercy, j'ai pu remarquer que les enchères atteintes pour un lot étaient moins fonction de la situation géographique et de la « valence écologique » de la coupe que de la position sociale des adjudicataires : les plus forts loyers étant payés par des tendeurs localement désignés par le terme de bricoleurs, c'est-àdire braconniers et/ou contrebandiers, comme si le groupe voulait économiquement sanctionner leur marginalisme et la déviation qu'ils sont censés faire subir à la tenderie en tentant de la rentabiliser ${ }^{14}$.

Ainsi, le contrôle et la sélection pratiqués par les tendeurs semblent opérer à deux niveaux hiérarchiquement organisés :

- au niveau de la commune où, par les mesures inscrites dans le cahier des charges, délibérées et adoptées en séance du conseil municipal, la tenderie devient un monopole communal, la commune s'opposant comme unité écologique et sociologique à l'extérieur ;

- au niveau intracommunal où, par le système des enchères, la tenderie se distribue socialement selon le degré de conformité reconnu ou postulé des partenaires, les tendeurs s'opposant en tant que groupe aux autres, aux non-tendeurs. \&Quel que soit le vainqueur du défi, note $\mathrm{J}$. Baudrillard, la fonction essentielle de l'enchère est l'institution d'une communauté de privilégiés se définissant comme tels par la spéculation agonistique autour d'un. corpus restreint de signes. La compétition de type aristocratique scelle leur parité (qui n'a rien à voir avec l'égalité formelle de la concurrence économique) et donc leur privilège collectif de caste par rapport à tous les autres, dont les sépare non plus leur pouvoir d'achat, mais l'acte collectif et somptuaire de production et d'échange de valeurs/signe ${ }^{15}$. \$

14. La tenderie n'est en effet pas considérée comme une activité lucrative : - On ne tend pas pour vendre (cf. infra).

15. J. BAUDRILLARD, Pour une critique de l'économie politique du signe, op. cit., 1972, p. 135. 
Le choix de la grivière passe donc nécessairement par le jeu de rapports sociaux inscrits dans un cadre géographique et sociologique restreint, et définis par le système de l'adjudication aux enchères publiques qui constitue les acteurs en groupe de a privilégiés (d'ailleurs idéologiquement représenté puisque les tendeurs se reconnaissent comme privilégiés en voyant dans la tenderie un privilège accordé aux habitants du plateau par le traité de Nimègue de 1678). Cela explique dans une certaine mesure que les conditions écologiques soient si peu envisagées par les tendeurs pour établir et/ou pour se représenter leurs grivières : selon eux, celles-ci sont également bonnes techniquement, leur valeur restant déterminée en dernière instance par le système d'échange (adjudication) auxquelles elles sont liées. Si d'un point de vue technique, comme l'ont montré les travaux de Lescouet (1947 et 1963) et d'Estivant (1962), \& les versants exposés au nord et au nord-est présentent de meilleurs conditions que les versants exposés au sud et à l'ouest ${ }^{16} \$$, et si les grives « prennent généralement mieux sur les hauteurs qu'en bas $\gg$, les tendeurs ne tiennent apparemment pas compte de ces conditions. La ruse, la finesse du tracé du sentier, la manière d'installer les pliettes et les hayettes et de poser les lacs garantissent plus que la situation géographique le « rendement » de la grivière. Il est d'ailleurs remarquable de noter que le tendeur n'attribue jamais la pénurie de captures à la situation de sa grivière, mais pratiquement toujours aux conditions météorologiques. En somme, et quel que soit l'emplacement de celle-ci, la logique de la représentation veut que, dès le moment où elle est adjugée, elle est supposée \& rendre \$. Il y a deux raisons qui déterminent cette attitude : d'une part, la confiscation des informations par les autres tendeurs ou leur déformation (surenchère, sousévaluation délibérées : dès que les grives arrivent, « on commence à mentir, à truquer les chiffres. S'ils sont gros, on les grossit. Médiocres, on les diminue.. Ça ne regarde personne ${ }^{17}$ ) ne permettent pas d'effectuer des comparaisons utilisables pour envisager le rapport; d'autre part, le système des enchères interdit implicitement de le retenir comme fonctionnel dans la mesure où il supposerait pour ce faire un truquage du jeu au départ, par la différenciation non contrôlée et a priori posée des lots selon leur valeur d'usage, donc selon le rendement postulé. Le système serait alors organisé sur la

16. J. LescoueT, \& La Tenderie aux grives \$, art. cité, 1963, p. 12.

17. M. Françors, « Le Tendeur et les saisons $\$$, art. cité, 1963, p. 7. 
base de la loi de l'offre et de la demande \& avec approximation maximale de la valeur d'échange et de la valeur d'usage », et non plus sur la base de l'enchère où « la valeur d'usage escomptée ne crôit pas au fur et à mesure de l'enchère ${ }^{18}$. $\gg$ L'enchère suppose une équivalence au départ de la valeur d'usage des lots. C'est finalement moins la matérialité fonctionnelle du lot que sa généalogie, c'est-à-dire le cycle de ses locataires successifs, qui détermine sa valeur d'échange.

Le pourcentage de tendeurs par rapport à la population totale de chaque commune dépasse rarement les $5 \%$. Cela pose déjà un problème car, malgré les affirmations répétées des tendeurs, il ne semble pas que ce pourcentage ait été plus important dans le passé. Si l'on prend l'exemple de Hercy, le $\mathrm{Dr}$ Séjournet ${ }^{19}$ estimait à cent le nombre de tendeurs en 1900 . Ce nombre représentait, sur la base du recensement de 1901, 8,3 \% de la population, donc un pourcentage relativement proche de celui de 1968 (7\%). Il y a certes eu plus de tendeurs dans le passé quactuellement, mais cette importance numérique devait être dans une certaine mesure fonction de celle de la population et du nombre de coupes disponibles. Si le nombre diminue en valeur absolue, il se stabilise par contre en valeur relative (pourcentage), et cela peut s'expliquer statistiquement par la dépopulation de plus en plus marquée des communes de la vallée de la Meuse et du plateau ardennais. Cela ne rend cependant pas compte du phénomène sociologique que représente cette relative stabilité. La situation à Hercy en 1900 est à ce titre exemplaire, car elle se présente - et est représentée - comme la « capitale de la tenderie ${ }^{20}$. Or, le pourcentage de tendeurs en 1900 était à $1 \%$ près le même qu'en 1968, alors que les contraintes et menaces pesant sur cette pratique y étaient moins sensibles. Un tel phénomène peut laisser supposer qu'il existe, à un niveau qu'il conviendrait de préciser, un contrôle social effectif dont le principal effet est d'établir un seuil démographique maximal, au-delà duquel la situation deviendrait aberrante, voire menaçante; en d'autres termes, limitant l'accès à la tenderie, donc le nombre des tendeurs. Ce n'est certainement pas au niveau administratif qu'il faut rechercher des facteurs de régu-

18. J. BAUDRILLARD, op. cit., 1972, p. 133-134.

19. La Grive dans les Ardennes... \$, art. cité, 1904.

20. La part du revenu communal que représente l'adjudication des lots de tenderie est la plus forte du département. Pour la période 1968-1973, elle est de $2080 \mathrm{~F}$ par an. Comparer avec Hautey, où elle n'est que de $300 \mathrm{~F}$ pour moitié moins de tendeurs. 
lation, puisqu'en théorie tout le monde peut tendre, mais plutôt aux niveaux sociologique et idéologique. Nous essaierons plus loin d'apporter quelques éléments de réponse à ce problème qui est sans aucun doute fondamental.

Le personnel de la tenderie est principalement constitué par des personnes ayant un âge au moins égal ou supérieur à quarante ans, exerçant une activité professionnelle au niveau communal (artisans, commerçants, bûcherons, cultivateurs, fonctionnaires communaux) et/ou aux horaires suffisamment autocontrôlés (artisans, bûcherons, cultivateurs) ou souples (ouvriers en équipes), ou n'ayant plus ou pas d'activité professionnelle (retraités, sans profession, femmes).

Les catégories sociales impliquées semblent donc limitées et déterminées par une condition fondamentale de la pratique de la tenderie : la disponibilité partielle ou totale de l'emploi du temps, qui constitue ainsi un facteur objectif de limitation à son accès. La préparation, la relève des captures notamment, qui exige durant les deux mois de l' « ouverture » un parcours quotidien de 10 à 30 kilomètres selon l'importance de la grivière et selon la longueur du sentier, ne peuvent être faites que par des personnes dont le temps n'est pas figé dans un cadre horaire rigide. Cette limitation n'est cependant pas impérative. Des tentatives ont été faites pour adapter la situation professionnelle aux nécessités techniques de la tenderie. Les trois ouvriers communaux de Hercy se sont « associés \ et ont arrangé leurs congés annuels de manière à ce que chacun d'entre eux ait le temps de « courir » sa tenderie et celles des deux autres; ils les ont donc étalés sur les trois mois (septembre, octobre, novembre). Cette tentative est en fait une véritable innovation sociologique dont les conséquences tant pratiques que structurelles sont capitales : d'une part, elle propose un mode d'organisation de la production qui annule sociologiquement l'effet sélectif du temps de travail en répartissant une opération clé, la relève des captures, sur des agents différents et dans un temps différent - à l'inverse du schéma traditionnel où un même agent contrôle dans un même temps une même opération. Les implications sociales de cette innovation sont justement de fournir une grille, un modèle d'organisation parfaitement adaptable aux conditions modernes du travail salarié, et donc de garantir la « survie \& de la tenderie, généralement contestée par ces conditions. D'autre part, il s'agit d'une association de type informel entre tendeurs de position sociale identique (ouvriers communaux), dans laquelle joue une forme de coopératon occasionnelle (relève 
des captures), techniquement non marquée - puisque l'un se substitue à l'autre dans le procès de production - mais sociologiquement pertinente et progressiste dans la mesure où : 1) elle suppose une forte cohésion interne ; 2) elle implique un partage des captures, donc un échange minimal d'informations; 3) elle dépasse le cadre traditionnel de la famille où jouent des rapports de coopération verticaux (essentiellement d'ordre technique : répartition de tâches différentes sur des agents différents dans un même temps) ; enfin 4) elle définit des rapports sociaux de production de type égalitaire par la délégation réciproque du contrôle social exercé sur la tenderie.

Ce groupe de tendeurs est le seul et unique que nous ayons rencontré lors de l'enquête, et il ne semble pas qu'il y ait eu d'autres tentatives de ce genre dans le passé. Bien que l'association existe depuis une dizaine d'années, l'exemple n'a pas été suivi par les autres tendeurs qui auraient plutôt tendance à le rejeter comme modèle d'organisation, considérant qu'il s'agit là d'une « industrialisation » de la tenderie (ils emploient d'ailleurs le terme de trust). Il semble en effet que l'existence de ce groupe, par le modèle de coopération qu'il propose (et qui contient en germe la répartition des frais d'adjudication), porte structurellement atteinte au contrôle individuel qu'exerce chaque tendeur sur une partie du territoire communal et sur l'ensemble des opérations techniques et économiques du piégeage. Le fait capital que les informations circulent parmi ces «néo-tendeurs \$, notamment et principalement celles concernant le nombre de captures réalisé, sape un des fondements du pouvoir des tendeurs qui se caractérise précisément par le contrôle absolu qu'ils effectuent sur la circulation et la distribution des grives : chaque tendeur choisit finalement sa clientèle. Ce pouvoir de sélection est obtenu et renforcé par le silence observé sur le nombre quotidien de prises et par le respect scrupuleux de circuits de distribution privilégiés. L'importante demande locale, voire régionale, en partie due à la connotation prestigieuse et somptuaire de la grive, favorise certainement le pouvoir social des tendeurs en accordant à leur position une fonction de prestige : capturer et distribuer les grives. Il semble d'ailleurs que les tendeurs jouent de cette situation et, en quelque sorte, la contrôlent en organisant une rareté borizontale au niveau de la distribution et en bloquant en amont les informations. Beaucoup d'informateurs font remarquer qu'une grive est d'autant meilleure qu'elle est inattendue. $\mathrm{Ce}$ groupe de tendeurs constitue bien une menace par l'élargissement sinon de fait, du moins possible, de la clientèle, que détermine la 
circulation même restreinte des informations. La critique portée par les tendeurs «classiques » paraît donc idéologiquement fondée : il peut s'agir d'une forme d'industrialisation et il est bien évident que les gens, sachant où se trouvent les grives, n'hésiteront pas à abandonner leurs «fournisseurs » attitrés pour s'en procurer. Le cas, aux dires de certains tendeurs de cette commune, s'est déjà produit ${ }^{21}$.

Un autre facteur limitatif, donc sélectif, découle directement des conditions imposées par le temps de travail : il sagit, comme nous l'avons vu, de la résidence sur le territoire communal. Le conseil municipal en a d'ailleurs fait une condition préalable à l'adjudication, comme le stipule l'article 3 du cahier des charges de Hercy : \& $\mathrm{Ne}$ seront admis à prendre part aux adjudications que les habitants domiciliés dans la commune au $1^{\text {er }}$ janvier 1968 sans distinction de nationalité. » La même clause se retrouve dans le cahier des charges de Hautey, article 3 : « L'adjudicataire devra être habitant de Hautey et titulaire d'un permis de chasse pris à Hautey. \Ces restrictions intéressent plus l'adjudication proprement dite que la pratique de la tenderie elle-même, mais elles sont cependant révélatrices d'un comportement global qui vise à faire de la tenderie un privilège des habitants de la commune (cf. supra).

En dernier lieu, lâge intervient comme critère distinctif, donc comme facteur limitant aussi l'accès à la tenderie. La corrélation notée entre l'âge du tendeur et sa position de retraité est structurellement accidentelle, bien qu'historiquement fondée et idéologiquement affirmée. Il est bien évident que, dans ce cas, lâge ne constitue pas un critère autonome. Il est plutôt un épiphénomène, voire une redondance du critère catégoriel. C'est bien là caractériser les réponses obtenues lorsqu'on faisait remarquer que les tendeurs se recrutaient principalement parmi les personnes âgées : "Les tendeurs sont vieux parce qu'ils sont retraités. \$ Or, le faible pourcentage de tendeurs dans les classes d'âge $20-40$ ans, le fait que dans le passé, c'est-à-dire à une époque où la retraite n'était alors qu'un vague

21. Il est assez surprenant de constater que le caractère informel et diffus du groupe des tendeurs ne l'ait pas empêché d'avoir une grande influence locale. D'une certaine façon, il y a une anomalie, voire une contradiction, entre la forte compétition interne, l'absence de coopération et les faits dé solidarité qui se sont manifestés en quelques occasions. En somme, et nous y reviendrons dans le chapitre suivant, la position de tendeur renvoie à des groupes plus larges, notamment fondés sur des rapports de parenté, et en reflète les contradictions et tensions internes. Cela n'empêche pas qu'en cas de conflits ils puissent les masquer. 
projet, les tendeurs, aux dires de la plupart des informateurs, étaient des personnes âgées permettent d'avancer que la tenderie est un privilège de la vieillesse. Certains vieux tendeurs font remarquer qu'à l'époque antérieure à 1914 les jeunes de 15-20 ans au plus, dont ils faisaient partie, tendaient aussi, mais ils précisaient toujours que cétait alors sous la direction du père ou d'un proche parent, l'indépendance technique ne s'obtenant qu'après rupture brutale de l'ordre familial : soit par le départ des jeunes tendeurs, soit par la mort du parent. Le fait que ce privilège se soit récemment incorporé dans une catégorie, celle des retraités, n'est qu'une conséquence de ce trait structurel. En ce qui concerne la tenderie, le contrôle technique ne pouvait être acquis qu'au prix d'une décharge des activités économiques du ménage ou de la famille sur les membres les plus jeunes, soit les enfants. En outre, l'acquisition des crins se faisait alors par prélèvement direct sur le cheval (de race ardennaise) que possédait chaque famille et qui était la propriété exclusive de son chef. De la même façon, le contrôle économique, fondé par l'adjudication des lots de tenderie, ne pouvait s'acquérir que par le pouvoir de disposer des ressources de la famille. La distribution des grives était également du ressort du chef de famille. Ainsi, contrôlé en amont et en aval, le procès de la tenderie dépendait entièrement de lui, donc et en théorie du plus vieux à la fois biologiquement et socialement.

Les grives capturées sont journellement distribuées. Le tendeur organise dans un premier temps son stock de captures sur la base de la distinction " grives données/grives vendues》, suivant leurs caractères spécifiques et leur mode de capture (à terre, à la branche). Les « vendues 》 sont généralement les draînes, litornes, merles (noirs et à plastron). Les "données 》, musiciennes, mauvis, sont quant à elles subdivisées en « données-données » et en « données-vendues \$ selon leur mode de capture. En outre, il semble que cette distinction première et générale ne soit pas aussi systématique et qu'elle se résume en définitive à une catégorisation de type négatif : " celles qui ne peuvent être données 》 (autorisant la vente des « données » non seulement en fonction de critères techniques de capture, mais aussi en cas de surplus), et excluant le don des \& vendues > qui constituent alors une catégorie parfaitement définie et homogène. Cette assertion n'est cependant valable qu'au niveau synchronique, car les catégories peuvent diachroniquement s'interpénétrer, voir s'inverser. Voici un exemple : E.P., tendeur à Hautey, donne huit grives \& parmi les 
plus valables , (roussettes prises à l'arbre), à raison de deux par semaine, à la femme du frère de la mère de son épouse. Au bout du quatrième don, soit de la huitième grive, cette dernière refuse d'en recevoir plus, sinon en les payant. E. P., ne voulant pas lui vendre des « données », lui offre des merles (« vendues ») qu'elle accepte (« données $»$ ).

En plus de l'inversion des catégories - les \& données > devenant \& vendues $>$ au niveau du receveur, et les « vendues \& se transformant en \& données \ au niveau du donneur - , une telle situation laisse apparaître un seuil de prestations d'une catégorie de grive au-delà duquel la contre-prestation simultanée en monnaie doit intervenir, à moins de passer à une autre catégorie (merle) dont la prestation semble illimitée en nombre ${ }^{22}$. Elle laisse également transparaittre la position d'autorité qu'occupe dans ce cas le receveur, puisqu'il accepte un lot précis (huit grives) sans fournir de contre-prestation. J'ai pu observer deux autres cas de ce type ; l'un concernait la sceur de l'épouse, l'autre la mère de l'épouse. Je ne suis pas en mesure de rendre compte de cette limitation, ni d'affirmer qu'il s'agit là d'un modèle de comportement traditionnel, encore moins d'une structure de distribution, d'autant que pour d'autres cas relevés le schéma était différent pour des positions structurales comparables des receveurs (vente et non plus don), mais conservait cette priorité de distribution aux alliés du tendeur. L'état actuel des informations ne permet pas non plus d'avancer que la différenciation entre \& grive donnée > et - grive vendue $>$ recoupe une division sociologique qui renverrait aux relations structurales du tendeur à ces groupes définis, mais les exemples rapportés plus haut en autorisent l'hypothèse.

Comme le suggèrent les précédentes observations, les grives sont distribuées en premier lieu aux alliés du tendeur, c'est-à-dire à la famille de la femme et, semble-t-il, quelle que soit la position qu'occupent les membres (c'est moins la distance structurale que le fait de relation qui importe). Ensuite, à la famille des femmes des fils ou à celle des maris des filles ainsi qu'aux maternels du tendeur (parents et frères de la mère, sœurs et enfants de sœurs), pratiquement jamais aux paternels (parents, frères et sœurs de père); enfin, aux relations de voisinage ( amis de maison $\gg$, amis de jardins $\gg$ ) et/ou aux relations professionnelles ou amicales (compagnons de régiment et/ou de déportation, camarades de jeunesse, etc.).

22. Catégorie connue et reconnue par les non-tendeurs (cf. supra, section II). 
La vente aux « étrangers \& - ceux qui n'entretiennent pas de relations sociales avec le tendeur - n'intervient qu'au terme de l'épuisement du circuit, après la part réservée à la consommation personnelle. Cela explique la rareté des grives mises en circulation dans les villes, sur les marchés. Le tendeur approvisionne d'abord la commune et vend le surplus aux étrangers à la commune. Ce comportement s'est notamment manifesté lors de la campagne de 1971 ; les marchés, les restaurants, les charcutiers et les marchands de gibier des villes de la vallée de la Meuse (surtout Charleville-Mézières, d'ordinaire mieux approvisionnée) n'ont pas « présentés \ de grives du fait du bilan désastreux de la saison. Comme me l'a dit un tendeur, * on a d'abord servi nos gens, et après cela il ne restait plus rien pour les autres ». En fait, il semble que la pénurie de grives, dont certains citadins se plaignent, se soit installée depuis cinq ans : les plantations d'épineux qui transforment l'écologie du plateau et détruisent le biotope de la grive en asséchant le sol et en ombrant les couverts, l'interdiction de la tenderie en Belgique (1967) où s'approvisionnaient les revendeurs citadins - en raison du surplus dont les Belges disposaient grâce aux tenderies au filet, interdites dans les Ardennes françaises - , la récente introduction du congélateur chez les tendeurs qui leur permet ainsi de \& prolonger \$ les circuits de distribution, ont sans doute été pour beaucoup dans son apparition et son maintien. Ces facteurs tendent à provoquer un resserrement en même temps qu'une simplification des circuits, qui devraient en permettre une meilleure lecture : la grive tend à circuler essentiellement au niveau communal.

Dans de nombreux cas, la femme du tendeur (ou le tendeur luimême) tient une comptabilité serrée des dons et des ventes sur un carnet dit de vente, avec indication des noms de personnes à qui les grives sont distribuées. L'artitude qui consiste à dissimuler ces carnets de vente traduit d'une certaine façon l'importance donnée à ces canaux de distribution qui doivent rester secrets sous peine de rompre l'équilibre, comme nous le verrons plus loin. Elle témoigne en outre des discordances possibles entre les comportements réels et le comportement normalisé, reconnu et avoué par le modèle des préséances volontiers tracées. Le respect de celles-ci semble en effet impératif et s'observe notamment dans les situations conflictuelles : j'ai eu connaissance d'un cas où le tendeur, par suite de pressions et de menus services rendus par son oncle maternel, l'avait servi en premier. La mère de la femme, l'ayant appris, rompit brutalement les relations 
avec son gendre en invoquant son égoïsme : il préférait sa famille à celle de sa femme, et en imaginant \& les malheurs que sa fille devait endurer dans les pattes de cette famille... ». Le conflit ne put se résorber que par un doublement des prestations lorsque les captures le permirent.

De telles \& histoires 》. sont assez fréquentes et contraignent le tendeur au respect scrupuleux du modèle, du moins en apparence, de même qu'au blocage des informations (compte tenu des pressions auxquelles il est constamment soumis par les uns et par les autres, et des situations qui l'obligent à rompre l'ordre des distributions), sous peine de voir sa clientèle fuir, son prestige faiblir et ses relations familiales se détériorer.

La répartition selon le modèle décrit plus haut n'est pas journalière, bien que les grives circulent quotidiennement, mais semble jouer à l'intérieur d'une unité de temps fondée sur la semaine, du moins en ce qui concerne la répartition au sein du réseau de parenté. La part en est limitée : que les grives soient données ou vendues, elle se fait sur la base de l'équivalence de deux grives pour un homme et d'une grive pour une femme. Quelle que soit par ailleurs l'importance des captures, la famille est approvisionnée en fonction du nombre de personnes par unité de résidence, or les parts ne peuvent dépasser douze grives en une seule fois. Le fait que, dans les exemples rapportés plus haut, la composition des dons n'obéisse pas rigoureusement aux règles de répartition par sexe - puisque la femme reçoit deux grives au lieu d'une comme le voudrait la règle - peut s'expliquer d'une part par le veuvage, d'autre part et surtout par une norme constatée mais encore inexpliquée qui interdit de donner ou de vendre une seule grive ${ }^{23}$. Celle-ci s'observe également dans le mode d'acquisition du sorbier : à Hautey, les sorbiers sont dorénavant estimés en paniers; l'équivalence d'une grive pour un panier de sorbes cueillies reste purement théorique, puisque les paniers sont reconvertis en bottées dont la valeur est définie par l'équivalent monétaire de deux grives. Ainsi la distribution des grives se fait au moins par paire, le nombre supérieur n'ayant à ma connaissance aucune importance symbolique (qu'il soit pair ou impair n'importe pas) bien qu'il ne puisse pas dépasser douze grives.

Les personnes étrangères aux circuits traditionnels de distribution

23. Cela ne contredit pas la loi de répartition par sexe, les femmes étant toujours en relation avec un homme, frère, mari ou fils. 
ne peuvent théoriquement obtenir des grives que par la médiation de ces mêmes circuits : il est d'usage, ai-je souvent entendu dire, qu'on ne vienne pas demander des grives directement au tendeur; on s'adresse à ses parents qui représentent dès lors le centre de distribution et de redistribution. La tenderie semble ainsi contrôlée par un réseau social dont le pôle serait constitué par les alliés et maternels du. tendeur qui lui délégueraient le pouvoir technique de capture des grives, sur la distribution desquelles ils ont un droit prioritaire de préhension. De ce fait, le nombre de tendeurs se détermine et s'autorégule en fonction des groupes familiaux en présence, un tendeur devant théoriquement suffire par génération de collatéraux. Cette hypothèse pourrait rendre compte du pourcentage relativement constant du nombre de tendeurs par rapport à la population totale de chaque commune et de l'impact socio-politique de cette pratique.

La distribution n'est cependant pas le seul mode de circulation des grives : elles peuvent intervenir comme monnaie d'échange de biens (cas du sorbier ou quelquefois des queues de cheval servant à la fabrication des collets) ou de services (dépannage technique, aide aux travaux de jardinage de construction, etc.). Le paiement en grives est parfois exigé à titre de dédommagement ou d'arrangement : N.M., tendeur à Hautey, demandait à J. V., autre tendeur, le paiement de grives contre un droit de passage sur sa tenderie. Des petits conflits (notamment des insultes) survenant fréquemment lors des parties de belote du samedi soir peuvent se régler en grives, même en dehors de la période de la tenderie, l'offensant acquittant sa dette à la prochaine saison. Il ne fait aucun doute que la valeur de "replâtrage » ainsi accordée à la grive se prête à des manipulations qui peuvent se traduire par l'insertion d'un nouvel élément dans la « sphère d'échanges $\gg$ (cas des «créanciers 》 offensés) et compromettre ainsi son équilibre par le bouleversement possible des préséances.

Le mode de distribution et d'échange des grives lui confère finalement une valeur eurythmique : il barmonise, simplifie en solidifiant, cristallise les relations sociales. A cet égard, la tenderie aux grives chez les Ardennais du plateau aurait une fonction de cohésion sociale, de réactualisation des rapports sociaux fondamentaux, en redéfinissant, par la répartition de son produit, leur nature, leur importance, leur étendue : cela au moment stratégique (automne) qui précède leur possible dissolution, en tout cas leur mise entre parenthèses (hiver), 
lorsque la rigueur du climat fermait et réduisait le champ de la pratique sociale, du fait que la neige et le verglas rendaient difficiles les communications avec l'extérieur. Il s'agirait en quelque sorte d'une " remise à neuf périodique de ces rapports \$, d'une * vérirable mise en scène sociale $\gg$, d'une institution dont la fonction serait à la fois consensuelle et différenciatrice; consensuelle dans la mesure où, par la ritualisation de la consommation de la grive, elle relie tous les habitants du plateau en une même communauté morale et une même collectivité distincte : les mangeurs de grives cuites d'une certaine manière; différenciatrice puisque, par les circuits ordonnés de distribution et d'échange, elle découpe, délimite des groupes en fonction de leurs rapports structuraux. En ce sens, la tenderie pourrait être définie comme une technique-rituel dont le tendeur serait l'officiant délégué. Dans le passé, les deux mois de la tenderie avaient l'allure d'une quasi-fête et se caractérisaient par une relative suspension du déroulement de la vie quotidienne et par le réajustement de la pratique sociale sur un mode structural. Chacun, en fonction de sa position, participait à cette activité. La vie du village était quasiment centrée sur la tenderie. Les cérémonies religieuses du dimanche étaient désertées par les hommes, les enterrements peu suivis, les mariages différés. J. Rogissart fait dire à un de ses personnages qui tente d'expliquer à une orpheline qu'il y aura peu de monde à l'enterrement de son père : \& Le matin, presque personne ne le suivra. En plein début des tenderies, ne crois pas que les hommes [...] Il vaudrait mieux les vêpres [...] Il faut comprendre Héloïse. Les grives doivent être ramassées le plus tôt possible chaque jour. Même pour les noces, on ne dérogerait pas à cette obligation. On y perdrait trop. Cest pour cela qu'on ne se marie jamais qu'après le 15 novembre ${ }^{44}$. ,

Que ce soit par cette intervention et par cette cassure qu'ils opèrent dans l'ordinaire de la vie villageoise ou par le contrôle qu'ils exercent sur le territoire communal, ou encore par la mobilisation de forces politiques constituées qu'ils peuvent déclencher (cf. le conflit de 1935), le pouvoir communal des tendeurs est indéniable. Pouvoir dans l'ombre certes; pouvoir secret, habilement dissimulé derrière un savoir technique et économique (la distribution) qui en conditionne l'exercice et en définit le niveau. La marginalité volontiers affirmée, et souvent défendue, joue comme leurre. Elle est là pour masquer et tromper - face aux pouvoirs \& officiels $\gg$ - pour

24. J. Rogissard, Passantes d'octobre, op. cit., 1958, p. 124. 
préserver peut-être une problématique communale et une indépendance souvent compromises et ébranlées. La proximité de la frontière belge, artificiellement tracée, l'ancienneté et l'importance de la contrebande (tabac, café) et du braconnage, les trois guerres qui, en moins d'un siècle, ont fortement éprouvé le département, etc., tous ces facteurs ont sans doute contribué à développer et à mettre au point, sur le plan des comportements, toute une stratégie de la dissimulation et de la rétention.

Ces conditions et ces contraintes de l'environnement socio-historique ne peuvent cependant, à elles seules, rendre compte des formes et modes de transmission des savoirs ou, d'une façon plus générale, de la problématique de la communication sociale chez les Ardennais du plateau. Elles en accusent sans doute certains traits et, de ce fait, les particularisent, mais elles n'en déterminent ni la structure ni la fonction, celles-ci paraissant liées à des logiques sociales plus profondes ou plus élémentaires. Les mécanismes de rétention du savoir, la constitution des secrets, les « lois du silence $\gg$ - négatifs de la communication mais peut-être aussi conditions - renvoient à la distribution sociale des savoirs et des compétences, à une problématique et à des rapports de pouvoir. 


\section{LA PRISE DE LA PAROLE DANS LA SOCIÉTÉ LIGNAGÈRE}

\section{Le soupçon et l'accusation}

Le passage d'un continent à l'autre ne traduit pas, contre toute attente, un changement radical de perspectives. $\mathrm{La}$ démarche et l'itinéraire restent les mêmes. Ici, on cache les pièges et les captures, on retient et on truque les mots - là-bas, on cache les parures et les masques (ce qui en soi dissimule déjà), on détient des secrets et on falsifie les voix; toujours sous le couvert de la forêt, qu'elle soit profane ou sacrée. Si dans les Ardennes les «lois du silence \$ sont finalement diffuses et peu articulées, en Afrique elles s'institutionnalisent pour marquer et démarquer (peut-être pour masquer) des positions et des attirudes. Dans les sociétés lignagères, « la loi sociale, écrit $M$. Augé, est une loi du silence qui désigne ceux qui ont le droit de la transgresser : les pouvoirs - bénéfiques et maléfiques, mais seuls leurs effets publiquement estimés permettent de les qualifier s'héritent selon des lignes préférentielles qui sont tout à la fois des lignes de force et des lignées sociales ${ }^{1}$ ». La dialectique du silence et de la parole, du nommé et de l'innommé, du soupçon et de l'accusation imprègne, en des lieux et temps sociaux définis, la situa-

1. M. AUGE, op. cit., 1974, p. 69. 
tion, la conduite et le destin social de l'individu ${ }^{2}$ en le confrontant aux pouvoir-dire et aux devoir-taire, en d'autres termes à une problématique et à une idéologie des pouvoirs. Silence et parole ne sont jamais neutres - ils ne sont pas plus sauvages qu'il n'y a d'hommes sauvages. Parler, c'est toujours s'exposer dans le mouvement même des réponses, comme le remarque J. Rabain-Zempléni : chez les Wolof, * les questions directes sont traditionnellement indiscrètes, incorrectes, voire ressenties comme agressives, non seulement entre personnes d'âge différent mais aussi entre égaux. En particulier, toutes les questions qui tendraient à mettre en évidence la bonne santé, l'intelligence, la prospérité sont considérées comme dangereuses parce que risquant de susciter la jalousie, l'envie et partant des pratiques de maraboutage et de sorcellerie. La " langue " est responsable de la mort, des maladies, des catastrophes. Si celui qui ne parle pas est mauvais, il y a une parole qui tue et qui déshonore ${ }^{3} \gg$. A la limite, elle serait menace, rupture, facteur de déséquilibre, remise en cause d'un ordre dont le silence serait métaphoriquement le porte-parole 4 . Pourtant, l'énonciation, la nomination, voire l'accusation, ont souvent valeur de replâtrage : elles dégèlent des situations de tension en repérant et canalisant les culpabilités ou les agressions réelles ou imaginaires. Le même auteur ajoute plus loin : « Les imputations d'agressions et les invites à la nomination semblent avoir précisément pour fonction la rupture, la dissipation de l'immobilisation, de la stagnation dont parle Lacan, la substitution à l'autre imaginaire de la situation d'agression de l'autre social ${ }^{\mathbf{5}}$. \$ Cette double articulation sociale de la parole n'est toutefois pas aussi différenciée qu'elle paraît à première vue. La fonction de replâtrage qu'on lui accorde souvent ne se fait pas sans bavures. Puisqu'elle est, en ce cas, remise en ordre, elle s'accompagne d'une certaine violence, donc d'une répression, en remettant à leur place agresseur et agressé et en évacuant au besoin, physiquement ou socialement, le ou les coupables. Les exemples wolof sur lesquels se fonde l'analyse de J. Rabain-Zempléni ${ }^{\circ}$ sont à ce titre doublement exemplaires : étant « au ras du sol » du quotidien et s'inscrivant dans le processus sourd de la socialisation de l'enfant en bas âge, ils montrent comment, en des lieux sociaux pourtant diffus, opère la fonction

2. Cf. aussi F. MICHELJONES, in M. AugE, ibid., p. 43 et s.

3. J. RABAIN-ZEMPLENI, art. cité, 1974, p. 152.

4. C'est peut-être ici et en ces termes que se pose la question du consensus.

5. J. RABAIN-ZBMPLENI, art. cité, 1974, p. 161. C'est nous qui soulignons.

6. 1bid., p. 157-160. 
répressive, par là même structurante, voire institutionnalisante, de la parole. Elle désigne les positions au sein de la structure lignagère et traduit l'ordonnance de leurs relations. A partir de situations d'agression et des réponses émotives de l'enfant, J. Rabain-Zempléni remarque, d'une part, que son questionnement par l'adulte - \& qui te l'a fait? - vise moins à « expliciter la nature du dommage et du conflit », qui peuvent être par ailleurs déjà reconnus, qu’à « refroidir le cour chaud de l'enfant \$, en déchargeant son imaginaire et ses fantasmes d'agression par le biais de la nomination qui en permet les repères et la réalisation sociale; d'autre part, que la nomination et la désignation par l'adulte - « c'est $x$ qui te l'a fait $»$-, venant en quelque sorte redoubler celles de l'enfant, constituent, soulignent et confirment l'ordre lignager, notamment par l'identification de l'agresseur à l'aîné, qui se voit du même coup investi du rôle de consolateur et de protecteur ${ }^{8}$; ainsi \& là où l'enfant est menacé de se trouver seul face à lui-même, à son malaise, à son mal-être, la parole de la mère introduit un rapport social, le rapport d'aîné à cadet ${ }^{\circ} \gg$. La prise en compte et la prise en charge de l'imaginaire de l'enfant, sa déconnection par l'invite à la nomination qui le situe au ras des rapports sociaux dénotent assez bien la fonction oppressive de la parole : elle charge (au propre et au figuré) les positions et les relations sociales.

Ces observations tendent à montrer que les mécanismes de partition et de répartition sociales, les rapports de subordination entre aînés et cadets se constituent et se renforcent, par le jeu du silence et de la parole, à des niveaux sociaux et pédagogiques élémentaires, non institutionnels, en tout cas antérieurs à l'initiation (quand celle-ci vient, ils sont déjà là), lors de situations quotidiennes, dans la cour ou la maison, presque a dans les jupes de la mère ». Cela confirme d'une certaine manière les remarques de $P$. Bourdieu, lorsqu'il parle de la persuasion clandestine d'une pédagogie implicite, capable d'inculquer toute une cosmologie, une éthique, une métaphysique, une politique, à travers des injonctions aussi insignifiantes que " tienstoi droit " ou " ne tiens pas ton couteau de la main gauche ". Toute

7. C'est nous qui soulignons.

8. L'attribution à la position d'aîné du rôle d'agresseur/protecteur est courante en Afrique de l'Ouest. Elle pourrait traduire cette * dimension totalitaire \ des sociétés lignagères, que soutient Augé, par cet assujettissement quasi total et souvent observé (cf. les travaux de P.-P. Rey et de C. Meillassoux) des cadets aux aînés sociaux.

9. J. RABAN-ZEMPLÉNI, art. cité, 1974, p. 160. 
la ruse de la raison pédagogique, ajoute-t-il, réside précisément dans le fait d'extorquer l'essentiel sous apparence d'exiger l'insignifiant ${ }^{20}$. Elles permettent en outre de repenser la période du « premier âge » de l'enfant africain, souvent négligée et abusivement envisagée en termes de corps à corps et d'indifférenciation sociale - l'initiation étant alors, dans cette perspective, surinvestie et survalorisée dans sa fonction de détermination sociale et culturelle -, en insistant sur la mise en place et en forme des structures et des comportements, ce qui, en d'autres termes, élargit le champ pédagogique des sociétés africaines et multiplie les lieux sociaux de leur reproduction.

$\mathrm{L}_{\mathrm{a}}$ fonction distributive et régulatrice de la parole ne doit, cependant, pas faire oublier les manipulations et les appropriations dont elle peut être l'objet. Un autre exemple, pris chez les Sénoufo du nord de la Côte-d'Ivoire, pose la question de son articulation à l'assise et à la position sociales des locuteurs, en un lieu qui est cette fois celui de la reproduction biologique et de ses représentations. Chez les Kiembara (sous-ethnie sénoufo de la région de Korhogo), tout rapport de parenté et d'alliance s'inscrit, se définit et s'organise dans le cadre idéologique du sandogo a d'esprit $\gg$ : appareil protecteur imaginaire du stock rituel de chaque narigba (matrilignage) appelé yésur, codé et contrôlé par les ancêtres (kulubélé) du lignage. Il s'incorpore socialement dans le sandogo "devin $" \mathbf{~}$, dont la fonction apparente est de lire et de repérer les facteurs de déséquilibre, notamment provoqués par tout rapport sexuel, potentiel ou réel, vu comme souillure (fonron). Cela se traduit et se manifeste au sein du narigba par la maladie interne et intériorisée (tiéfuru), c'est-à-dire non lisible sur le plan anatomique - auquel cas les techniques thérapeutiques traditionnelles seraient d'abord utilisées -, qui atteint l'enfant en bas âge ou la femme fécondable, dès lors appelés fonronučko (victimes du fonron). En accord avec le chef de lignage (narigbafolo) qui détient, par sa proximité généalogique avec le monde des ancêtres, le savoir des composantes et exigences du sandogo d'esprit, il recherche la coupable et fixe le moment et les modalités de l'action purificatrice (yapèré), dont le taux (čikpagi) est défini une fois pour toutes dans chaque lignage. Il est estimé en cauris qui sont déposés dans le vase du sandogo (sandogi) conservé par le chef du matrili-

10. P. BOURDIEU, Esquisse d'une tbéorie de la pratique, op. cit., 1972, p. 197.

11. Appelé en franco-ivoirien cbarlatan; presque toujours une femme chez les Kiembara, qui subit une initiation particulière. 
gnage; et/ou en poules, moutons ou chèvres, égorgés par la plus vieille femme du lignage, ménopausée (donc sexuellement neutre et neutralisée, symboliquement pure), sur le sandö̈, pierre ou ensemble de pierres situées à l'extérieur du village, à la limite des terres du narigba : actions qui rééquilibrent et font recouvrir au sandogo d'esprit son efficacité symbolique. Le sandogo apparaît donc comme un principe - une puissance que les devins peuvent décoder mais non contrôler - issu des ancêtres, qui régularise et protège les rapports au sein du narigba. La répartition et la régulation des dires et des agirs semblent ici fonction du statut social, précisément de la place occupée dans le matrilignage. La maîtrise conceptuelle que le devin a théoriquement des opérations lignagères ne lui confère pas de pouvoir-dire, puisqu'en dernier ressort c'est le chef de lignage qui désigne et accuse ${ }^{12}$. Il est en somme tenu au silence. Cela ne l'empêche pas toutefois de détenir un certain pouvoir, mais qui s'exprime d'une manière négative : il ne peut être accusé ni d'agressions ni de sorcellerie. Le halo de silence qui entoure la pratique du devin et la détention des secrets qui la fonde, acquis lors de l'initiation au sandogo, agissent en quelque sorte comme \& garde-fous ». Les devins sont d'ailleurs plus ou moins mis à l'abri et à l'écart : l'autel des ancêtres du lignage (kulukpagi) et celui des ancêtres sandobélé (sandoi) constituent généralement deux lieux de culte séparés - le premier au centre du quartier (katiolo), à l'intérieur du village, le second à la périphérie, aux limites du terroir —, comme si les devins, qui n'ont ni le droit de cultiver la terre ni celui de faire la cuisine pour leurs maris (n'oublions pas que, chez les Kiembara, ce sont surtout des femmes), étaient retirés de la chaîne généalogique et formaient un groupe à part, rejeté du côté de la brousse, donc alliés aux n'débélé, sorte de génies, détenteurs et fournisseurs des connaissances et des pratiques thérapeutiques, qui sont précisément les propriétaires mystiques de la terre, ceux à qui il faut payer par des sacrifices le droit de la cultiver. Cette quasi-alliance des sandobélé aux n'débélé tend à montrer que la souillure, d'origine sexuelle, est non seulement perçue comme perturbation des rapports à l'intérieur du lignage, mais aussi de ceux avec la terre. Elle renvoie à deux chaînes de références idéologiques, les ancêtres et la problématique de la descendance, les

12. A rapprocher de la remarque que font Adler et Zempléni : « Assurément, il y a quelque contraste entre ce statut social médiocre du devin et l'importance de sa pratique dans la vie rituelle de la communauté et des affaires privées \(L\& Bâton de l'aveugle.., op. cit., 1972, p. 47). 
《 génies de la brousse et celle de la terre; en fait à deux types de savoirs, généalogique et mystique, qui ne sont finalement articulés que par le pouvoir du chef de lignage ${ }^{13}$. La catégorie de sandogo d'esprit ${ }^{14}$, sa représentation, sa fonction de protection et son appropriation tant symbolique qu'économique par le narigbafolo traduisent bien cette ingérence du lignage dans les « affaires de la brousse $\gg$, de même que l'allégeance des devins à son autorité. Il s'agit pour cette dernière de contrôler l'usage de leur savoir. L'exigence de sens n'est, en effet, pas toujours satisfaite par le devin qui, également guérisseur (savoir «transmis » par les n'débélé), tente parfois de court-circuiter la logique interprétative en faisant valoir son pouvoir thérapeutique. Mais, dans ce cas, la parole du chef de lignage prévaut et le diagnostic voulu est alors recherché ailleurs, chez un autre devin. En ce sens, la pratique divinatoire ne ferait que confirmer ici la politique lignagère : le pouvoir mystique des devins doit être conjuré et leur puissance mise au pas de l'ordre lignager.

En définitive, il doit se jouer du côté des frontières du terroir villageois un combat douteux entre les kulubélé (ancêtres) et les n'débélé, dont les premières victimes sont les femmes et les enfants du lignage; les unes pouvant être "pourfendues » par le sexe des n'débélé d'une puissance et d'une longueur démesurées, \& avec lequel ils s'entourent les reins »; les autres pouvant être le fruit de cette mésalliance. L'agression sexuelle que traduisent, au niveau du village, l'adultère et les rapports sexuels non institutionnalisés défie le \& patrimoine génétique $\gg$ et foncier du matrilignage en compromettant la descendance et la filiation de même que le mode d'attribution et de répartition des terres dont la femme est le vecteur privilégié ${ }^{15}$. La participation soupconnée des devins à ce « combat», notamment attestée par les interdits qui marquent leur statut (ne pas cultiver la terre, ne pas faire la cuisine) et singulièrement symbolisée par les plaisanteries et insultes à caractère sexuel qu'ils font sur l'assistance masculine lors des funérailles de l'un d'entre eux, nécessite en quelque sorte leur détachement du stock généalogique « ordinaire \& et leur

13. Chez les Kiembara, j'ai observé que le savoir du devin, la valeur de son diagnostic sont souvent contestés, ce qui expliquerait la fréquence des consultations, les déplacements et la mouvance de la clientèle. Il n'est pas rare que certains fassent jusqu'à 50 kilomètres pour consulter.

14. Qui n'existe pas, par contre, chez les Nafara, autre sous-ethnie importante voisine des Kiembara.

15. Cf. L. ROUSSEL, Région de Korbogo..., rapport sociologique, op. cit., 1965 , p. 61-72. 
mise à distance de la vie active \$. Ils sont écartés pour être, au besoin, mieux repérés et mieux récupérés.

Sur un autre plan, la mise en scène de certains masques traduit également cette coalition des deux univers qui orientent et bornent l'idéologie sénoufo : celui des règles, du corps social, du village et des champs, le kulo ; et celui des virtualités, des puissances inconnues et incontrôlées, de la brousse et des franges, le duninian, le « grand univers ». Les masques wabélé, abondamment produits et justement célèbres par leur forme singulière - taillés dans la masse, ils représentent grossièrement une tête de bovidé pourvue d'immenses mâchoires, proéminentes, pouvant atteindre jusqu'à un mètre de long -, participent de ce dernier et sont perçus comme des instruments d'agression : « irruption 》 des potentialités du \& grand univers $>$ dans celui des hommes... Ils ont 4 la réputation de disposer de pouvoirs prodigieux et toujours nuisibles : provoquer la maladie, l'infirmité, la stérilité, inciter la femme d'autrui à commettre l'adultère, tuer ${ }^{16} \gg$. Leur puissance maléfique est tout entière contenue dans une boule de matière, enroulée dans une feuille (war) et placée sur le front du masque ou à l'intérieur des mâchoires. Seul le propriétaire individuel ou collectif (wabafolo) du masque détient le secret de ses composantes, de ses actions et de son pouvoir. Agissant en dehors du cadre initiatique (ce qui explique dans une certaine mesure leur 'prolifération), leur manipulation n'est en principe soumise à aucune règle. Les porteurs subissent une initiation courte et rudimentaire, calquant, singeant même celle du poro, et peuvent intervenir à tout moment dans la vie publique, apportant une violence symbolique (perturbations et désordres dans le déroulement d'un rituel) ou réelle (menaces, lancer de haches sur les chiens, quelquefois sur l'assistance) au sein du corps social, qui se défend \$ alors, selon une logique manichéenne mais dans un temps différé, par l'intermédiaire d'un autre masque, à la puissance bénéfique et protectrice, le kurubla. Proche dans sa forme des wabélé, il ne s'en distingue souvent que par des bouquets de plumes d'aigle pêcheur fichés sur la mâchoire supérieure et sur le haut du masque. Bien que théoriquement indépendant lui aussi de lorganisation initiatique, il est presque toujours placé sous le contrôle des chefs de bois sacrés (sinzangfolo) et se substitue ou succède au kpéligé (masque propre au poro) lors du rituel funéraire, dans la phase du \& chevauchement

16. G. Bochit, Les Masques sénoufo... •, art cité, 1965, p. 654. 
du cadavre $\gg:$ :Alors que les wabélé n'interviennent en général dans le domaine funéraire que pour le décès de l'un de leurs agrégés, le korobla monte sur ce lit [le lit funéraire] de la même façon que le gpelige et contribue, non plus par un bartement de tambour mais par un cri, qui est singulier, à chasser l'âme (ple) vers le séjour des morts (kubelekaha) ${ }^{17}$. $\gg$ Le manichéisme sénoufo, le dualisme des univers et des forces associées, symbolisées ici par les wabélé et les kurubla, ne sont pas aussi clairs et nets. L'affrontement joué par les masques ne leur confère pas toujours une connotation définie, permanente et signifiante, appréhendable dans leur forme ou dans leurs évolutions, qui les rejetterait de part et d'autre d'une frontière rigide que la pratique sociale rend justement floue et mouvante. La fonction protectrice du kurubla est quelquefois gauchie par une mise en scène spectaculaire, recourant à la surenchère, aux procédés, voire aux supercheries, qui terrifie l'assistance et qui le tend menaçant, redoutable dans cette lutte imaginaire avec les forces maléfiques (il crache du feu dans un accompagnement sourd de tambours et de vrombissements); la puissance et la magie d'agression des wabélé ne sont pas aussi a sauvages et imprévues que le dit l'idéologie sénoufo. Elles sont en somme déjà socialisées et socialisables, contrôlées et contrôlables, et cela se repère notamment dans la fonction disciplinaire qui leur est quelquefois accordée : sous la forme télékuru, les wabélé sont les - garants pour les serments graves : ils tueront le parjure ${ }^{18}$ \$.

La relative identité des formes wabélé et kurubla, leur signification et leur fonction accrochées à des éléments mobiles, périphériques, surajoutés, par là même interchangeables (substance végétale, bouquets de plumes notamment), attestent bien l'ambiguïté de leur position dans lordre des représentations, la souplesse de leur utilisation dans l'ordre cérémoniel et leur caractère d'accessoires idéologiques, plutôt que de supports ${ }^{19}$. Quoique affirmée et abondamment commentée, personne ne croit fondamentalement à \& l'agression

17. Ibid., p. 661 .

18. Ibid., p. 657.

19. Cette disponibilité des formes est en tout cas étonnante chez les Sénoufo : ils n'assignent pas aux masques proprement dits un contenu idéologique spécifique et permanent. Tout paraît dépendre du contexte tant liturgique que rituel. En ce sens, je souscris entièrement aux thèses de $G$. Bochet : les masques sénoufo, * contrairement à ce que voudrait le préjugé nourri par les Européens à leur égard, non seulement ne jouent en aucun cas le rôle de supports idéologiques privilégiés, mais subissent en fin de compte un sort inattendu : ils disparaissent (art. cité, 1965, p. 676). > 
sauvage $\gg$ des wabélé (du moins parmi mes informateurs) : leur pouvoir terrifiant et maléfique réside manifestement dans cet espace du secret et dans cette loi du silence qui entourent leurs manipulations et leurs intentions. Etant théoriquement n'importe qui (puisque les règles d'utilisation ne sont pas socialement codées), ils peuvent s'en prendre à n'importe qui et créer de la sorte un climat de terreur, souvent noté par les observateurs, « qui envahit parfois la vie psychologique du Sénoufo jusqu'à l'étouffement ${ }^{20}$ 》. Il est d'ailleurs remarquable de noter que le porteur de wabélé est un homme « qui n'est pas désigné par la terminologie puisque seul le personnage existe $^{21} \gg$. Cette puissance diffuse, non assignable, en fait un instrument efficace d'oppression et de répression, précisément par cet anonymat qui se paie parfois le luxe d'une remise en ordre sous une apparence de désordre (d'où la fonction disciplinaire du télékuru). En conséquence, et bien qu'apparemment incontrôlés tant dans leurs actions, dans leur.production que dans le recrutement de leurs desservants, les wabélé n'en sont pas moins soumis, à l'instar des devins, à l'ordre lignager et à l'organisation initiatique qu'ils contribuent à reproduire ou à maintenir.

Ainsi, pour en revenir au propos initial, dès l'instant où une femme en âge de procréer ou un enfant en bas âge sont atteints d'un mal non éruptif (tiéfuru, ou kalama : « gros ventre »), le père, le mari ou le chef de lignage selon les cas, et surtout selon la résidence, consultent le devin afin de découvrir le ou les responsables du fonron qui en est l'origine et qui connote les rapports sexuels avant et hors mariage. La culpabilité de la femme est socialement négligée, mais c'est à elle ou à son père que reviennent la tâche et le devoir de payer le yapèré. S'il en était autrement, me confia un chef de lignage, si je dis à l'homme qui a connu la femme d'aller chercher les cauris ou la poule, j'en mourrai par les ancêtres parce que j'aurai donné la même femme à deux hommes ». Cette taxe fait, en effet, partie intégrante de la compensation matrimoniale ${ }^{22}$ que le jeune homme doit verser

20. G. BOCHET, art. cité, 1965, p. 641. J'ai fait des constatations semblables au début de mon enquête en pays sénoufo (cf. J. JaMm, La Nébuleuse du Koulo-Tyolo..., op. cit., 1973, p. 3 et s.).

21. Ibid., p. 655.

22. Taxe à laquelle s'ajoute le nčigi qui varie de 6000 à 8000 cauris payés au chef de lignage de la femme, et qui autorise le jeune homme à - partager le lit avec la femme $\gg$. Ces cauris sont conservés par le chef de lignage pour son usage personnel (surtout achat de pagnes funéraires), à l'inverse du čikpagi qu'il dépose dans le sandogi et qui serait récupéré à sa 
au chef de lignage de la femme dont il est preneur et vise à décharger la puissance perturbatrice du rapport sexuel conséquent : elle représente en somme une garantie et un affranchissement à l'égard du sandogo ${ }^{23}$. Ces attitudes et ce système de représentations semblent traduire une crainte, voire une angoisse, de la sexualité perçue ici comme désorganisation d'un ordre dont le sandogo est l'opérateur ${ }^{24}$, qui pourrait être concomitante d'une société matrilinéaire où la tentation sociale de l'inceste est forte puisque l'épouse sociale idéale est la sour (notamment dans le cas du kékourougou) ${ }^{25}$. Le rapport au mari ne peut être alors en théorie qu'un rapport sexuel, d'où son importance sociale et la nécessité de le contrôler sur un plan rituel et idéologique ${ }^{26}$. En fait, le mari ou « l'homme extérieur 》 peut, par ce rapport, et doit de toute manière perturber et compromettre l'équilibre et l'intégrité du yésur (appareil idéologique du matrilignage), par la constitution d'une descendance qui biologiquement échappe en partie à ce dernier - d'où l'attention quasi maniaque que

mort par son successeur pour l'accomplissement de sacrifices « veillant à son bon voyage et à sa bonne réception dans le monde des morts $\gg$.

23. Depuis quelque temps, et à la suite des secousses qui ont ébranlé les structures traditionnelles et notamment les modèles matrimoniaux, la valeur purificatrice du yapèré s'est institutionnalisée au point, chez les Kiembara, de désigner une forme de mariage, le yapèrétio, textuellement femme du yapèré. Selon mes informateurs, ce type d'union apparaîtrait dans les jeunes générations qui, voulant se choisir \& librement 》 comme partenaires sexuels, c'est-àdire en dehors de l'influence des vieux qui jusqu'alors maîtrisaient le mode de distribution et de répartition des femmes, par là même celui des terres, peuvent s'acquitter de la dette du sandogo par le versement unique de cauris et/ou d'offrandes de poules, de moutons, de chèvres au chef de lignage de la jeune fille. Bien que la jeune fille puisse alors quitter son narigba et se démarquer de sa destination matrimoniale originelle $\$$, le versement du yapèré (assez élevé dans ce cas : le taux n'est plus fixe) suffit. Concession sans doute faite par les vieux à une évolution irréversible et dont ils tirent de toute manière profit sur un plan idéologique et rituel, voire économique, même s'ils perdent en partie le contrôle de la descendance.

24. D'une façon générale, et sur un plan plus théorique, on peut se référer ici aux thèses de R. Girard : " La sexualité, écrit-il, est impure parce qu'elle se rapporte à la violence $\gg$ (La Violence et le Sacré, op. cit., 1972, p. 55-59).

25 . Il s'agit de cette forme singulière de mariage, avec résidence natolocale : le mari vient visiter son épouse chaque soir, mais retourne travailler le matin dans son village maternel. La descendance reste acquise au matrilignage de la femme. Cf. L. ROuSSEL, op. cit., 1965; A. MARIE, - Parenté, échange matrimonial et réciprocité... \$, art. cité, 1972, 2, p. 16-17; et J. JAMIN, La Nébuleuse de Koulo-Tyolo..., op. cit., 1973, p. 35-37.

26. C'est dans cette perspective que l'on peut interpréter toute la symbolique sexuelle des épreuves physiques et du matériel rituel du poro (cf. la description qu'en fait chez les Diéli G. Bochet, "Le Poro des Diéli 》, art. cité, 1959). 
les Kiembara fixent sur les maladies des enfants, indicatrices de la souillure. Il s'agit moins de guérir et de sauver l'enfant (ou la femme) que de guérir et de sauver l'équilibre du yésur, dont le sandogo constitue en quelque sorte la \& garde \& et la \& sonnette d'alarme \$.

D'autre part, ce système exprime le pouvoir de contrôle sexuel que les vieux, principalement les chefs de lignage, possèdent sur les femmes puisque ce sont eux qui en dernier ressort, après la mise en évidence du fonron, désignent la coupable réelle ou imaginaire. Ce pouvoir est toutefois et parfois curieusement contesté, davantage par la parole que dans la pratique : le discours tenu à certains moments par les neveux (fils de la sceur) paraît autoriser ces derniers à avoir des rapports sexuels avec la ou les femmes de leur oncle, même si celui-ci est encore vivant. Bien qu'elle puisse s'exprimer publiquement, voire en présence de l'oncle qui se contente d'acquiescer, de sourire, de bougonner ou de répliquer par des insultes rituelles (sorte de relation à plaisanterie appelé bari), une telle assertion est difficilement crédible, en tout cas invérifiable, même si la terminologie de parenté kiembara en mime la réalisation puisque les enfants de mon oncle sont mes enfants \& et sa femme « mon épouse \$ - elle m'appelle mon mari $\gg{ }^{27}$. En outre, ce comportement, s'il était vrai, remettrait en cause ce que nous avons exposé plus haut sur la représentation de l'adultère. Dès lors, expression sans doute d'un imaginaire catégoriel, on peut s'interroger sur la place des locuteurs dans le code des relations sexuelles et sur les registres matrimoniaux. Larticulation structurale de deux formes de mariage, diafotio et narbatio, de même que la répartition sociale et spatiale des lignes aînées et cadettes pourraient fournir un schéma d'interprétation.

Le diafotio ou tofotio (selon qu'ego est le père ou le fils) signifie la femme donnée au fils, ou la femme donnée par le père. Sur le plan normatif, le père donne de préférence sa nièce utérine (fille de la sceur) à son fils, ce qui a pour conséquence de réduire les termes de l'échange matrimonial puisque le frère donne sa scur et récupère la fille de sa sour qu'il donne à son fils (voir fig. 1) : ainsi le départ d'une femme d'une concession est donc compensé par la venue de sa fille, et cela traduit un cycle court du type $A \rightarrow B, B \rightarrow A$. Cette pratique permet au frère de contrôler, en plus de la descendance de sa sceur, celle de son fils qui réintègre le narigba (matrilignage) du grand-père, et de régulariser en quelque sorte la relation fils/neveu

27. Cf. J. Jamn, La Nébulouse du Koulo-Tyolo..., op. cit., 1973, p. 27-31. 
en les répartissant dans des concessions différentes. D'une certaine façon, il y aurait dans cette situation, et à un niveau structural, une - invasion » de la patrilinéarité puisque le fils du fils appartient au lignage du père du père, travaille sur ses terres, donc profite de leur produit et logiquement lui succède, étant le fils de la fille de la sœur du père du père, en quelque sorte et potentiellement un neveu du père du père : il hérite du frère de sa mère, qui n'est autre que le neveu utérin du père de son père.

En somme, l'autorité familiale qui échappe au père serait récupérée à l'intervalle d'une génération : le père, en donnant sa nièce utérine à son fils, cumule les fonctions sociales. d'oncle et de père (de ses petits-fils). Il y aurait donc une brèche dans le système matrilinéaire, conséquence de la patrilocalité, qui pourrait rendre compte du passage rapide de certaines sous-ethnies à la filiation et à la succession patrilinéaires. Le neveu n'est finalement qu'un relais - concession faites à l'ordre matrilinéaire - dont la position stratégique est accusée par le narbatio modèle 2 (voir fig. 3) : la possibilité d'épouser la veuve de son oncle ajoute à sa position structurale d'oncle maternel celle de « père \$ du fils du frère de sa mère, donc de grand-père paternel du fils de sa sœur - son neveu utérin devient son petit-fils.

Le narbatio modèle 1 (voir fig. 2) en est une conséquence structurelle, formulée ainsi par les Kiembara : Le père donne sa nièce à son fils, mais ne peut donner sa fille à son neveu. S Le mariage avec la cousine croisée matrilatérale impliquerait en effet l'interdiction du narbatio modèle 2 , dans la mesure où, en épousant la veuve de l'oncle maternel, le neveu épouserait la mère de sa femme! L'oncle peut donner à son neveu, dans la logique du système défini par le diafotio, la fille de la scur du mari de sa sœur (ou la fille du frère de sa femme); et sa fille au fils du frère de sa femme (ou au fils de la soeur du mari de sa sœur). Sur un plan structurel, ce mariage reproduit le diafotio : l'oncle donne finalement la nièce utérine du mari de sa sœur à son neveu.

Les deux formes d'union narbatio, bien que n'étant pas distinguées sur le plan lexical, sont sociologiquement pertinentes dans la mesure où leur pratique met en scène des acteurs sociaux différents qui sont dans la relation généalogique aînés/cadets. Le fait que l'oncle ait le droit de prélever une partie de la descendance de sa sœur - toujours les cadets, mais jamais les deux sexes en même temps - le contraint, en échange du travail fourni, de leur trouver des femmes. Le cadet se trouve dans un état de double dépendance : vis-à-vis de son oncle 


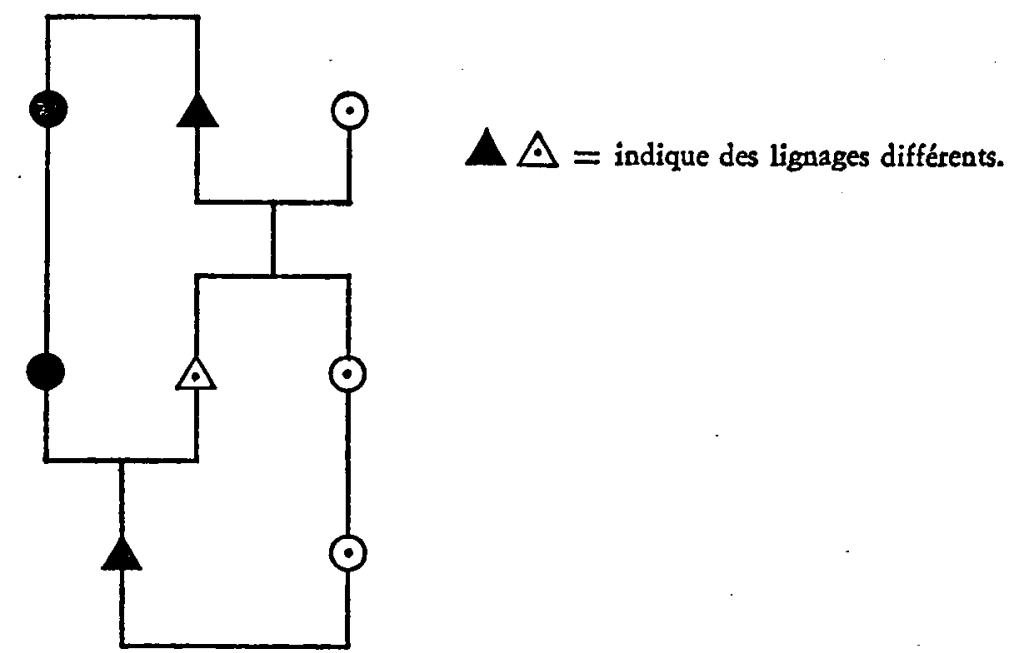

figure 1 : diafotio ou tofotio. Modèle théorique. Mariage préférentiel avec la cousine croisée patrilatérale.

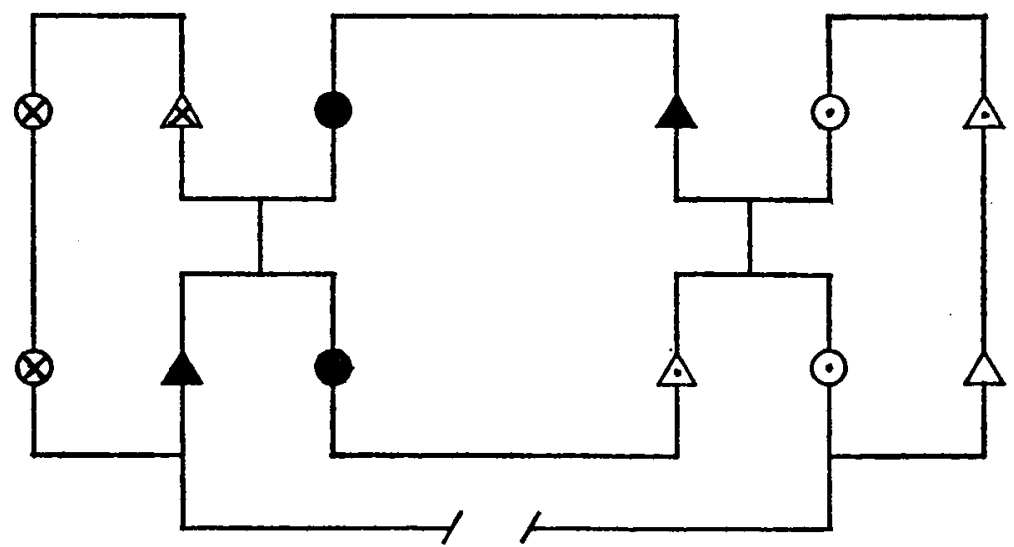

figure 2 : narbatio, modèle 1 . Interdiction de mariage avec la cousine croisée matrilatérale. 


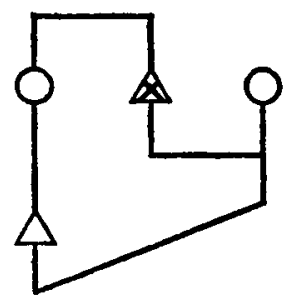

$\Delta=$ décéde.

figure 3 : narbatio, modèle 2. Mariage avec la veuve du frère de la mère.

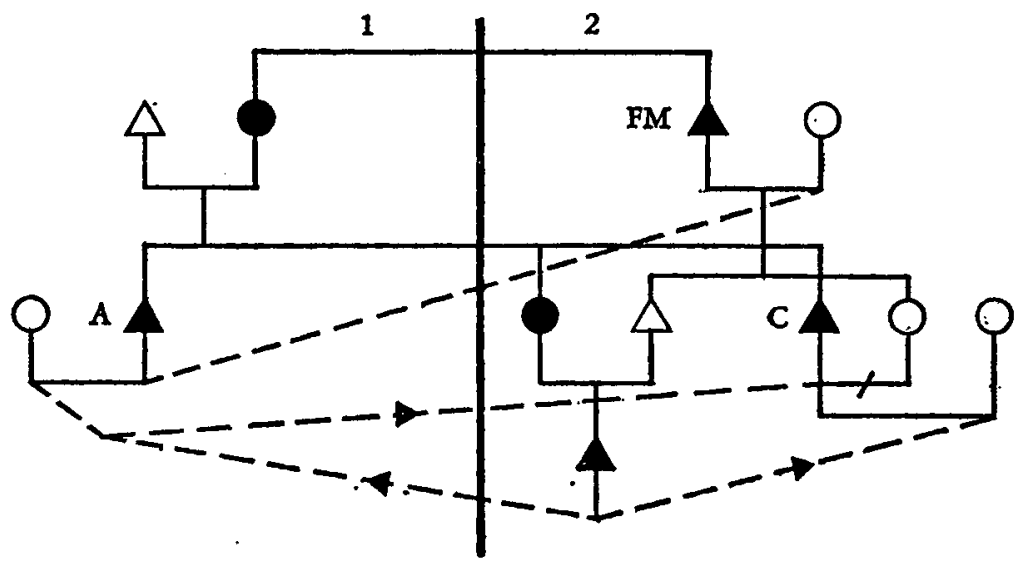

- $=$ limite de concession

- - - = mariage et rapports sexuels possibles. La flèche indique l'ordre théorique.

$+=$ mariage interdit

$\mathrm{FM}=$ oncle

$\mathrm{A}=$ aîne

C $=$ cadet

figure 4 
et vis-à-vis de son frère aîné. Ce dernier reste, par contre, avec le père jusqu'au décès de l'oncle maternel ${ }^{28}$, dont il occupe la place et peut recevoir la femme en héritage (cas du narbatio modèle 2). A la mort de l'aîné, le cadet hérite théoriquement de sa femme, de son statut et de ses biens, mais peut se trouver en compétition, sur le plan successoral, avec le petit-fils aîné de son oncle maternel : soit, suivant la logique du système diafotio, avec le neveu utérin de son frère aîné, c'est-à-dire et encore avec son propre neveu qui, en raison de la patrilocalité, réside dans la même concession et peut revendiquer la femme du frère aîné (narbatio modèle 2), celle de son oncle (voir fig. 4). Au niveau des attitudes, la relation de plaisanterie (bari) oncle/neveu suppose la proximité généalogique mais la distance géographique (l'oncle et l'aîné des neveux utérins vivent dans des concessions séparées, et de fait c'est l'aîné qui peut plaisanter l'oncle et qui tient ce discours sur ses femmes). Par contre, la distance généalogique et la promiscuité semblent provoquer des tensions : le cadet peut s'éliminer de la chaîne successorale par l'interdiction d'être promu en même temps que son aîné dans le même bois sacré ce qui implique, comme nous le verrons plus loin, une double résidence - , et voir de ce fait le petit-fils de son oncle maternel (son 《 neveu ») profiter de la perte momentanée mais répétée du contrôle qu'il pourrait avoir sur le jeu social à l'intérieur de la concession (encore que celui-ci existe jusqu'à l'âge d'entrée au poro). Ainsi, les positions théoriques des aînés et des cadets dans l'espace matrimonial défini par le diafotio et le narbatio nécessitent en raison de certains facteurs, notamment démographiques et géographiques, des redéfinitions et des réaffirmations constantes. Le discours tenu par les aînés à propos des femmes de l'oncle s'inscrit dans cette problématique : il peut être bien entendu une métaphore, une anticipation du narbatio modèle 2 , mais aussi et surtout une manière ludique de marquer leurs droits et leur place dans la chaîne successorale, face à une terminologie indifférenciée, qui associe donc aînés et cadets dans leurs rapports formels aux femmes de l'oncle - d'autant que ces derniers, par leur résidence théorique, côtoient quotidiennement

28. Qui est lui-même un cadet, puisqu'il hérite en principe de son frère aîné. Comme chez les Alladian, * le matrilignage définit les lignes de transmission de l'héritage : héritage du pouvoir politique et du pouvoir de gestion des biens communs qui se transmet horizontalement, de lignée a lignée, et non point toujours directement de l'oncle maternel au neveu utérin. (M. Augk, in C. Meillassoux, L'Esclavage en Afrique précoloniale, Maspero, Paris, 1975, p. 458). 
celles-ci : proches des femmes, proches de l'oncle, ils sont en tout cas menaçants par le savoir qu'ils détiennent, sans pouvoir l'exprimer, sur le jeu des relations à l'intérieur du katiolo (quartier) ${ }^{29}$. Le « partage sexuel \ ainsi revendiqué par l'aîné, et toléré publiquement dans sa formulation, introduit, pourrait-on dire, un partage social à l'intérieur d'une catégorie de parenté qui recoupe théoriquement un partage géographique, et qui pourrait avoir pour but de récupérer et de camoufler la distance par l'identité simulée et verbalement affirmée à l'oncle.

\section{Pouvoir de la parole et parole du pouvoir}

Pouvoir sur les autres, pouvoir de l'Autre, maléfique ou bénéfique, la représentation et le destin social de la parole paraissent en tout cas plus complexes que le suggère $P$. Clastres ${ }^{30}$. Elle peut, comme nous l'avons vu dans les exemples précédents, ordonner, réguler, mystifier, conjurer, séparer, etc.; mais il demeure, derrière ces représentations et ces connotations, une fonction répressive, voire oppressive, car de légitimation et d'accusation (au sens propre et figuré), qui la situe donc du côté des pouvoirs. A cette articulation volontiers affirmée, décrite et analysée dans la littérature ethnologique, à cette alliance entre pouvoir et parole, il faudrait ajouter aussi et peut-être surtout un troisième terme, celui-là négatif du deuxième, c'est-à-dire l'implicite, le non-dit, le devoir-taire, le silence. Si, pour reprendre l'expression de P. Clastres, la prise et l'exercice du pouvoir sont prise et exercice de parole, j'ajouterai qu'ils sont également jeu et gain de silence, et que le pouvoir ne peut s'acquérir et/ou se maintenir que par l'appropriation et, ainsi, que par la rétention de cette parole. Le chef, dans la société primitive, est certes le maître des mots, mais il est en même temps celui des silences et des secrets... Il est celui qui sait taire et se taire. Il n'est donc pas certain qu'un chef silencieux ne soit plus un chef ${ }^{\text {s1 }}$. On pourrait même renverser la proposition et suggérer que l'avancement dans la hiérarchie nécessite et implique un

29. Ils sont d'ailleurs souvent soupçonnés en cas de fonron.

30. La Société contre l'Etat, op. cit., 1974, p. 133-136, 161 et s.

31. lbid., p. 134. 
savoir-taire. En effet, ici peut-être plus qu'ailleurs, savoir tenir sa langue, c'est savoir tenir sa place et distribuer celle des autres. Cela pourrait être une de ces lois du silence qui autorise la confiscation et l'accumulation des savoir et des pouvoir-dire et qui implique la mise en œuvre de savoir et de devoir-taire.

Une recherche récente ${ }^{32}$ montre comment l'apprentissage de la parole peut être aussi apprentissage des silences, des pondérations et des rétentions. L'enfant wolof doit apprendre à se taire et à écouter, à ne pas dire n'importe quoi à n'importe qui n'importe où et n'importe quand, à avoir la langue courte. Il s'agit en somme de temporiser et de localiser la parole en l'incorporant aux statuts et aux rôles, de délimiter donc, d'une façon structurale, des zones de silence qui deviennent autant de sphères de pouvoir.

$A$ un niveau plus théorique, $O$. Ducrot, contestant la conception saussurienne de la langue comme code, comme simple transmission de l'information, et partant des thèses de la philosophie analytique anglaise, note que dans l'acte linguistique lui-même il y a « des thèmes entiers qui sont frappés d'interdits et protégés par une sorte de loi du silence (il y a des formes d'activité, des sentiments, des événements dont on ne parle pas). Bien plus, il y a, pour chaque locuteur, dans chaque situation particulière, différents types d'informations qu'il n'a pas le droit de donner, non qu'elles soient en elles-mêmes objets d'une prohibition, mais parce que l'acte de les donner constituerait une attitude considérée comme répréhensible [...] Dans la mesure où, malgré tout, il peut y avoir des raisons urgentes de parler de ces choses, il devient nécessaire d'avoir à sa disposition des modes d'expression implicite, qui permettent de laisser entendre sans encourir la responsabilité d'avoir dit $^{33}$ ». Dans les sociétés lignagères, un tel recours et une telle attitude apparaissent nettement lorsqu'il s'agit d'interpréter un événement marquant, mort ou maladie, celles-ci \& pouvant faire l'objet de diagnostics qui, à la suite de circonstances nouvelles, se corrigent, se compliquent ou s'infirment les uns les autres ${ }^{34} \gg$, sans pour autant compromettre la logique lignagère ou le discours du pouvoir, ceux-ci se donnant les moyens, par la loi du silence et par le jeu de l'implicite, de les cumuler et de les accumuler. La sauvegarde de la parole, de la compétence ou de la crédibilité nécessite alors soit l'utilisation de modes d'expression implicite (laisser entendre), soit

32. J. RABAIN-ZEMPIENI, op. cit., 1975, chap. 7, p. 409 et s.

33. O. Ducror, Dire et ne pas dire, op. cit., 1972, p. 5.

34. M. AUGE, op. cit., 1975 , p. 412. 
la délégation du dire à des instances marginales. C'est du moins ce que l'on peut inférer de l'exemple sénoufo rapporté plus haut, où le chef de lignage, bien qu'en définitive maître du diagnostic et de l'accusation, protège le pouvoir de sa parole en l'abritant derrière le savoir des devins, moins impliqués socialement et idéologiquement. L'acte de prendre la parole ne semble donc être \& ni un acte libre ni un acte gratuit. Il n'est pas libre, en ce sens que certaines conditions doivent être remplies pour qu'on ait le droit de parler, et de parler de telle ou telle façon. Il n'est pas gratuit, en ce sens que toute parole doit se présenter comme motivée, comme répondant à certains besoins ou visant certaines fins ${ }^{33} \gg$.

La parole vide et neutre dont parle P. Clastres ${ }^{38}$, qui caractériserait le discours du chef dans les sociétés primitives, me semble donc moins, comme il le dit, être une mise à distance, un refus du pouvoir qu'une opération de masquage du pouvoir, opération qui en situe ainsi l'origine et le fondement en dehors du corps et du verbe du chef. Etant donné que tout ce qui est dit peut être contredit, il est donc nécessaire, comme le fait remarquer $O$. Ducrot, « à toute croyance fondamentale, qu'il s'agisse d'une idéologie sociale ou d'un parti pris personnel, de trouver, si elle s'exprime, un moyen d'expression qui ne l'étale pas, qui n'en fasse pas un objet assignable et donc contestable ${ }^{37} \gg$. Si le chef a le devoir de parole, comme l'écrit P. Clastres, il parle sans se jouer, il parle au nom de... tout le problème est de savoir au nom de quoi et de qui.

Ces remarques faites, on peut mieux cerner la place des devins chez les Sénoufo Kiembara, dont la fonction, du moins dans le cas cité, reste imprécise puisqu'en fin de compte ils ne font que confirmer par leur diagnostic la « parole $>$ du chef de lignage. Il y a en somme, dans ce camouflage idéologique qui consiste à faire valider et publier, par la pratique divinatoire, le processus d'accusation, une décentration des lieux du pouvoir qui tend à mieux en protéger le corps : la concession apparemment faite au savoir et au pouvoir des devins (sans pour autant être absolue puisqu'on joue en fonction du but recherché de leur nombre et de leur dispersion, voire de leur rivalité) "défocalise 》 pratiquement les lignes de force en en replaçant l'origine du côté de la brousse ou du côté

35. O. Ducrot, op. cit., 1972, p. 8.

36. Op. cit., 1974 , p. 135.

37. O. Ducrot, op. cit., 1972, p. 6. 
des ancêtres. «Le chef, écrit G. Balandier, a l'obligation de manifester, à chaque instant, l'innocence de sa fonction ${ }^{38}$. $>$ Ce processus est bien connu et répond à cette économie du pouvoir qui consiste à accroître l'emprise en démultipliant les prises et en n'offrant pas de prise. Le pouvoir est en quelque sorte, ici, dissocié du corps par la mécanique des secrets qui entoure ses origines, ses fondements, son exercice et sa force. Il ne se voit pas ou peu, ne se montre pas, ne se dit pas ${ }^{30}$. C'est en fait tout l'appareil lignager qui produit du pouvoir et qui place les individus \& dans un champ permanent et continu > de significations et de relations. Ce qui ne veut pas dire, comme l'affirme rapidement $P$. Clastres ${ }^{10}$, que la parole du chef n'a pas force de loi : elle a secretement force de loi, dans ses détours et ses retraits ${ }^{41}$, dans son arrimage et ses reports à des réseaux symboliques qui en légitiment le sens et l'usage. Le discours du pouvoir lignager n'est ni autoritaire ni démonstratif. Il est totalisant et totalitaire, il n'y a pas d'un côté production massive d'idées par les maîtres, de l'autre petit bricolage conscient-inconscient de reprise et d'élaboration personnelles dans l'intimité du sous-exercice d'un logos privé, mais pratique sociale globale d'une fantasmatique sémiologique \$. M. Augé, dans un langage moins sophistiqué, moins - parisien », avance une thèse semblable à partir de l'étude des jeux de pouvoir dans les sociétés lagunaires de Côte-d'Ivoire. Posant la notion d'idéo-logique, définie comme logique des représentations, comme systématique virtuelle des représentations, il écrit : « L'idéo-

38. G. BALANDIER, Antbropologie politique, op. cit., 1967, p. 47.

39. Chez les Sénoufo, mais c'est également vrai d'autres sociétés africaines (cf. en particulier les travaux de P. Etienne sur les Baoulé), le pouvoir économique doit être dissimulé. Le bétail, signe de richesse, est dispersé dans de nombreux troupeaux de village et confié à la garde des bergers peul, qui de ce fait, mais en tant qu'étrangers, en connaissent l'importance et la valeur. Celles-ci sont en partie révélées à la mort du propriétaire, lors des funérailles, par l'héritier qui en sacrifie quelques têtes. Les pagnes funéraires sont, de même, secrètement conservés dans une valise ou une malle et exposés au moment des funérailles.

40. Op. cit., 1974, p. 176.

41. A noter que tout retrait, réserve, retenue, non-échange peuvent être interprétés comme manifestation de/du pouvoir. La proximité des vieillards et/ou des enfants (réincarnation) avec le monde des ancêtres leur confère un savoir sur la mort qui leur donne un pouvoir sur la vie. Cf. J. RABAINZEMPIENI, op. cit., 1975, p. 150; ainsi que A. ZEMPLENI et J. RABAIN, - L'Enfant nit $\mathrm{ku}$ bon. Un tableau psychopathologique traditionnel chez les Wolof et Lebou du Sénégal $\gg$, Psychopatbologie africaine, 1965, I, $\mathrm{a}^{\circ} 3$, p. $295-439$.

42. J. Frankln, Le Discours du pouvoir, op. cit., 1975, p. 201. 
logique comme discours théorique possible est unique, en ce sens qu'il n'y a pas un discours des chefs et un discours des non-chefs, un discours des aînés et un discours des cadets, un discours des hommes libres et un discours des captifs : un secteur ou une région de l'idéo-logique qui exprimerait le point de vue des dominants et un secteur ou une région qui exprimerait le point de vue des dominés [...] Elle est dans la pratique de tous ${ }^{25}$.

C'est peut-être dans la mise en scène et l'économie de la parole et du discours que réside « la ruse de la raison lignagère \$ : faire croire que le pouvoir est ailleurs, derrière les mots, dans l'ombre des bois sacrés, dans le secret des masques, toujours indéfini, toujours insituable, mais toujours présent. Les remarques de $\mathbf{M}$. Foucault sur le \& pouvoir disciplinaire » pourraient dans une certaine mesure s'appliquer à la réalité lignagère. Il observe en effet que le pouvoir disciplinaire peut être à la fois « absolument indiscret, puisqu'il est partout et toujours en éveil, qu'il ne laisse en principe aucune zone d'ombre et qu'il contrôle sans cesse ceux-là mêmes qui sont chargés de contrôler ; et absolument discret, car il fonctionne en permanence et pour une bonne part en silence. La discipline fait " marcher " un pouvoir relationnel qui se soutient lui-même par ses propres mécanismes et qui à l'éclat des manifestations substitue le jeu ininterrompu de regards calculés. Grâce aux techniques de surveillance, la " physique " du pouvoir, la prise sur le corps s'effectuent selon les lois de loptique et de la mécanique, selon tout un jeu d'espaces, de lignes, d'écrans, de faisceaux, de degrés, et sans recours, en principe au moins, à la force, à la violence ${ }^{4}$. Un tel rapprochement peut paraître hasardeux, démesuré ou impropre. Toutefois, le jeu des rituels et des épreuves initiatiques, le système des classes d'âge, celui des rangs, des degrés, des catégories d'âge, la distribution des savoirs, l'effet des regards, les lois du silence et le poids des secrets font malgré tout penser à une « organisation disciplinaire \ de la société lignagère ${ }^{\star s}$ : chacun est placé, situé, observé, protégé et

43. M. AUGE, op. cit., 1975 , p. 410.

44. M. FoucAuLT, Surveiller et punir..., op. cit., 1975, p. 172.

45. D'ailleurs, P. Clastres (op. cit., 1974, p. 152-153), malgré lui et d'une certaine façon contre lui, opère métaphoriquement ce rapprochement entre pouvoir disciplinaire et pouvoir lignager lorsqu'il ouvre son débat sur la torture dans les sociétés primitives, et notamment lors de l'initiảtion, par des citations de La Colonie pénitentiaire de Kafka et par une référence à l'expérience de Martchenko des camps soviétiques. Ironie du sort ou ruse de linconscient? 
agressé, en quelque sorte rangé et classé. Cette thèse peut surprendre, d'autant que des ouvrages récents semblent accorder aux sociétés traditionnelles et/ou sans Etat un savoir-faire social et un savoirvivre politique qui les protégeraient par essence des enfermements et des machines de pouvoir, de l'accumulation et de la possession. Mais ces sociétés sont-elles si conscientes du \& mal » de l'Etat ou du pouvoir au point de se définir contre lui ? L'égalitarisme, plus souvent affirmé que décrit, dont elles font preuve ne serait-il pas plutôt de l'ordre des discours, d'un certain type de discours qui aurait pour fonction non de défendre ou de préserver le communautaire et le collectif, mais de taire la division et la différence ? Discours dont l'occurrence, le sens et la pertinence paraissent d'une part varier suivant la position sociale du locuteur, d'autre part se réduire au fur et à mesure que l'on monte dans l'ordre hiérarchique, comme si le discours le mieux entendu et peut-être le plus efficace était celui qui n'est pas effectivement tenu. De ce fait, à s'en tenir aux discours \$ officiels \$, qui fonctionnent ici sur le mode économique du singulier-pluriel (on parle de quelques-uns pour tous), on risque fort de ne saisir que ceux dont ils parlent, c'est-à-dire ceux qui ont le droit à la parole, le pouvoir de parole, les a aînés $\gg$, les chefs, et de les prendre pour ceux à qui ils s'adressent, les « cadets », les sujets; d'occasionner ainsi un nivellement par le haut : la parole des uns devient la parole de tous, d'où l'homogénéité sociale souvent affirmée et postulée à partir de l'homogénéité de sens. Le fait que ces discours aient été pris au mot, que leur « silence » ou leur " neutralité \$ n’aient pas été systématiquement interrogés, explique peut-être la vision idéale, homogène et quelque peu rousseauiste que certains ont des sociétés primitives. Dans le chapitre suivant, nous tenterons d'analyser cette économie de la parole et du discours dans les sociétés lignagères en partant des lieux privilégiés de leur reproduction sociale et culturelle : les classes d'âge et les initiations. 


\section{III}

\section{SECRET INITIATIQUE ET POUVOIR LIGNAGER}

Il y a un paradoxe dans tout projet initiatique, puisqu'il tend à uniformiser en « humanisant $»$ et en * harmonisant $\gg$ - il s'agit de réaliser l'bomme tribal -, mais divise en socialisant : phase centrale et quasiment motrice de la reproduction sociale, l'initiation ne peut que reproduire les rapports de subordination et la structure hiérarchique de la société lignagère, même si elle les camoufle pour un temps derrière des rituels communs, dans l'inculcation d'un même savoir et dans l'organisation en associations et en classes d'âge. Entreprise de marquage, n'est-elle pas aussi processus de masquage ?

\section{Le silence des structures}

L'opposition classe d'âge/lignage est classique. La littérature ethnologique a souvent montré comment l'organisation de la société en classes d'âge tendait, par le recrutement, l'étendue, les faits de solidarité et les liens de pseudo-parenté qui y jouaient, à évacuer, du moins en apparence, la structure généalogique. Eisenstadt, dans une perspective fonctionnaliste, note qu' * un système quelconque de classes d'âge apparaît chaque fois que le milieu familial ne suffit pas à 
assurer, avec la pleine éducation de tous les membres de la société, la conservation de ses valeurs rituelles et morales ${ }^{1}$ ». D. Paulme reprend cette démarche en observant que « l'importance des classes d'âge se révèle inversement proportionnelle à celle des lignages, d'autant plus grande que la solidarité organique de ces derniers est moindre, leur extension limitée dans le temps et dans l'espace. De cette lutte contre la parenté, la classe d'âge n'est pas toujours victorieuse; il arrive que la solidarité de clan l'emporte sur celle de l'habitat ${ }^{2} \gg$. Ainsi se pose, suivant ces approches, la question de la fonction globale des classes d'âge, à la limite en termes de lutte contre la parenté, contre les lignages. Sans entrer dans un débat théorique qui nous éloignerait du propos, on voit mal cependant comment une société s'embarrasserait d'un dispositif sociologique aussi lourd et complexe pour pondérer ou évacuer une structure (le lignage) qui la fonde, qui persiste, qui paraît finalement bien s'en accommoder ; à moins que ce dispositif ne soit lui-même ou bien soumis à la loi lignagère auquel cas la structure du lignage opérerait une "coupe verticale * dans le système des classes d'âge (cas des systèmes dits cycliques) et rendrait compte de ce fait de leur hiérarchisation -, ou bien reproducteur de la structure lignagère - auquel cas l' égalitarisme 》 et la démocratie affirmés (promotion globale, \& roulement $>$ des générations au pouvoir) seraient quasiment un leurre, puisqu'en dernier lieu ce serait la position généalogique qui définirait le statut et le rapport au pouvoir. L'appréhension synchronique et la visée fonctionnaliste qui marquent la plupart des études consacrées aux classes d'âge ${ }^{3}$ paraissent occulter leur articulation et intrication à l'ordre lignager, en privilégiant d'une part leur organisation interne, d'autre part leurs rituels et fonctions, et en négligeant pour ainsi dire leur dynamique, leur évolution, leur destin social. Il faut toutefois reconnaître que ces systèmes se prêtent volontiers, par l'idéologie qu'ils véhiculent, à des interprétations en termes de dépassement, de remplacement et d'oblitération de la structure lignagère. Dans le temps et le lieu de la classe d'âge, celle-ci est en quelque sorte 《 silencieuse \$, du moins est-elle mise pour un moment « en veilleuse s. Ce qui peut donc relever d'une approche, description et analyse ethnologiques est en somme déjà inscrit dans la réalité : la

1. Prom Generation to Generation, Londres, 1956; cité par D. PAULME, Classes et associations d'âge..., op. cit., 1971, p. 9.

2. D. PAULME, op. cit., 1971, p. 16.

3. Je me réfère principalement aux études éditées par D. Paulme, ibid. 
classe d'âge se donne comme transcendant les rapports de parenté et d'alliance. Tout se passe comme si la socialisation des individus opérait en dehors de leur vécu social, dans une mise à l'écart parfois rituelle (que traduit assez bien l'enclos initiatique). L'exemple des classes d'âge kikuyu (Kenya) est à ce titre remarquable, d'autant qu'il fut popularisé et « gonflé » par les analyses de J. Kenyatta (1967) et par le mouvement Mau-Mau de 1952.

Selon J. Kenyatta, le système des degrés d'âge « contribue à cimenter l'ensemble de la tribu » que l'agencement en mbari (patrilignages) tend à faire éclater en groupes familiaux largement autonomes. Cet auteur ajoute plus loin : "Toute la société kikuyu repose sur la notion d'âge : cela est établi de façon telle que le plus petit enfant en a conscience. Il est banal de dire que toutes les obligations sociales, chez les Kikuyu, sont déterminées par le système des degrés d'âge, mais il est plus intéressant de noter que ce système prévaut dans tous les domaines, des plus futiles aux plus importants. Il règle la façon de saluer ou de manger certains aliments, la répartition des tâches, au foyer ou aux champs, la manière de s'habiller ou de se tenir en public : il explique le droit de juger ou d'exercer une autorité dans le clan ou la famille, il indique la place à occuper dans le déroulement des cérémonies *. " Le premier problème auquel se heurte l'observateur est sans aucun doute d'ordre terminologique, car les Kikuyu n'utilisent, en apparence, qu'un seul terme pour désigner les différentes unités de classification. Ce terme est morika ou muburika ${ }^{5}$, dont la forme pluriel est rika ou riika selon la graphie utilisée par Kenyatta ${ }^{\circ}$. Seul le contexte permet de juger à quel élément structurel il est fait allusion. Il est certain qu'il y a une part d'arbitraire dans le choix de nos concepts; toutefois, les renseignements fournis par la bibliographie, et notamment par A. Prins (1953), permettent d'avancer que les Kikuyu font eux-mêmes une distinction entre rika, qui désigne à la fois le groupe annuel de circoncision, soit la classe d'âge, ainsi que la génération définie dans ce cas comme « la période pendant laquelle le gouvernement est aux mains d'une des moitiés tribales ${ }^{7} \gg$; et murika (ou morika), qui désigne les groupes qui composent chacune des classes comme ces classes elles-mêmes.

4. J. KenYaTTA, Au pied du mont Kenya, op. cit., 1967, p. 85 .

5. C. W. HOBLeY, Bantu Beliefs and Magic, op. cit., 1938, p. 87.

6. Op. cit., 1967, p. 24.

7. A. Prins, East African Age-Class Systems..., op. cit., 1953, p. 41. 
Selon A. Prins ${ }^{\text {, }}$, ce terme désignerait par extension le degré d'âge (que J. Kenyatta nomme riika ${ }^{\circ}$ ). Compte tenu de ces remarques préliminaires, nous définirons donc la classe d'âge (rika) comme l'ensemble des jeunes Kikuyu circoncis une même année; les degrés d'âge (morika) comme les différents stades par lesquels passe une classe d'âge ou une partie de classe d'âge; et la catégorie d'âge (morika également) comme le groupe social occupant un degré d'âge donné.

Il y a théoriquement une cérémonie de l'initation chaque année, mais en raison de famines ou de guerres il peut arriver qu'il s'écoule un ou deux ans, parfois plus, entre deux cérémonies consécutives ${ }^{10}$. La totalité des hommes circoncis la même année forme une classe d'âge dénommée d'une manière uniforme, quels que soient la région ou le district. Le nom donné se réfère à un événement précis survenu pendant l'année. Les membres d'une classe d'âge sont des compagnons d'âge qui appartiennent à quatre classes groupées deux à deux. Un jeune initié appartient ainsi à l'une des deux moitiés tribales maina ou mwangi, selon un principe que nous examinerons plus loin, et à l'une des deux confréries ou associations de circoncision, à l'origine différenciées par l'opération chirurgicale, soit kuruitha ukabi ou \& mode masaï \$ (incision du filer) et kuruitha ukikuyu ou a mode kikuyu $\gg$ (circoncision) (voir fig. 5). Comme le fait remarquer $C$. W. Hobley ${ }^{11}$, c'est moins la façon dont les garçons sont opérés (puisque cette opération est actuellement la même pour les deux confréries, soit l'incision du filet) que le rituel de circoncision qui distingue les associations. Ce rituel déterminera les rituels ultérieurs de même que les rites de purification en cas de thabu (souillure). L'appartenance à une des associations ne semble avoir aucun effet pertinent sur la structure du système social et n'a de toute manière aucun rapport avec la division en moitiés tribales. L'appartenance à l'association tribale est déterminée par la filiation patrilinéaire, et on peut supposer que la division résulte de la détérioration progressive du système clanique et constitue peut-être une simplification de même qu'une extension de son modèle à l'ensemble de la tribu. L'appartenance à une des moitiés tribales est, quant à

8. Ibid., p. 42.

9. Op. cit., 1967, p. 143.

10. Cf. C. CAGNOLO, The Akikuyu..., op. cit., 1933, p. 198.

11. Op. cit., 1938 , p. 80 . 


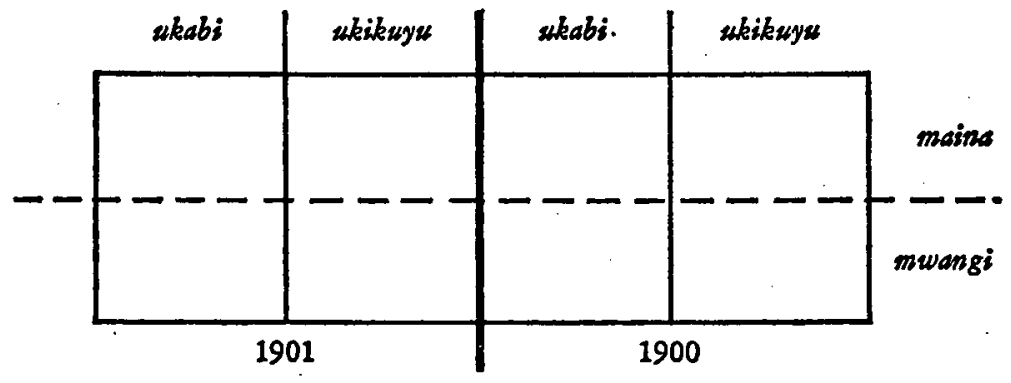

figure 5

(d'après Prinis)

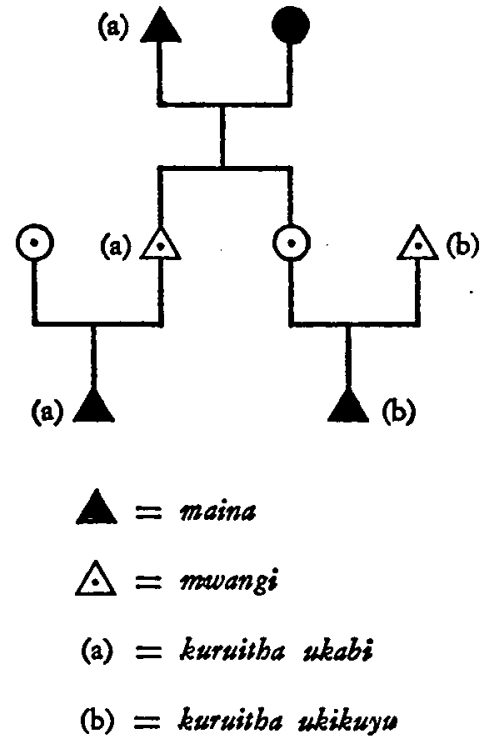

figure 6 
elle, déterminée par l'alternance des générations patrilinéaires (voir fig. 6).

On doit l'expression « moitié tribale » à A. Prins (1953). Jusqu'aux travaux de cet auteur, les différents observateurs avaient pris le terme de génération dans son sens strict, c'est-à-dire avaient pensé le système en termes de générations biologiques successives. Cette interprétation est, cependant, bien embarrassante pour résoudre le problème posé par la promotion individuelle que représente l'accès au kiama kia mataatbi ${ }^{12}$, qui recrute ses membres au fur et à mesure, et par la promotion collective que représente le \& roulement $\$$, l'alternance des générations au pouvoir tous les vingt-cinq ou trente ans. $\mathrm{Si}$, en fait, on s'en tient à l'idée de générations biologiques successives, il devrait y avoir nécessairement une période pendant laquelle il n'y aurait pas de gens présentant les conditions requises pour être investis du statut d'ancien du kiama kia mataatbi. Ces anomalies disparaissent si, comme A. Prins, on fait une hypothèse toute différente, et que l'on suppose donc que les générations kikuyu sont des générations sociologiques contemporaines. Selon A. Prins, le processus d'alternance des générations se ferait de cette façon : « la délégation de l'autorité politique détenue par une moitié de la plus vieille des classes d'âge tous les trente ans aux membres les plus vieux de lautre moitié tribale. Ainsi, il est facile de comprendre que la période de gouvernement de chacune des moitiés se pense en termes de générations institutionnelles $^{13} \gg$. Les termes mwangi et maina se réfèrent donc et uniquement aux générations vivantes, l'une étant au degré de ce que $A$. Prins appelle * anciens-gouvernants $\gg$ (génération au pouvoir), l'autre étant à celui d' « anciens-consultants » (génération alterne). Les générations qui disparaissent reçoivent un nom défini traditionnellement par les tâches qu'elles ont réalisées. Le système semble donc semi-cyclique. On peut illustrer cela en affectant à chacune des générations les lettres $A, A^{\prime}, B, B^{\prime}$, etc., qui symbolisent leur nom propre, et en mettant en vis-à-vis, suivant un ordre indiqué par les dernières générations et suivant le principe de l'alternance, leur nom structurel, c'est-à-dire mwangi ou maina. On aura ainsi :

$$
\begin{aligned}
& \mathrm{A}=\text { mwangi } \\
& \mathrm{A}^{\prime}=\text { maina }
\end{aligned}
$$

12. Cf. J. KenYatTa, op. cit., 1967, p. 140. C'est ce conseil qui détient le pouvoir politique.

13. A. PRINS, op. cit., 1953, p. 43. 


$$
\begin{aligned}
& \mathrm{B}=\text { mwan }_{\mathrm{b}^{\circ}} \\
& \mathrm{B}^{\prime}=\text { maina } \\
& \mathrm{C}=\text { mwangi } \\
& \mathrm{C}^{\prime}=\text { maina } \\
& \mathrm{D}=\text { mwangi } \\
& \mathrm{D}^{\prime}=\text { maina } \\
& \mathrm{E}=\text { mwangi } \\
& \mathrm{E}^{\prime}=\text { muirungu (ou mwirungu) - maina }
\end{aligned}
$$

Du point de vue tribal, la génération se réfère donc à la période du passé pendant laquelle les anciens de l'une ou l'autre des moitiés tribales tiennent les rênes du gouvernement et administrent la justice. Ainsi, pendant les générations $\mathrm{A}, \mathrm{B}, \mathrm{C}$, les anciens mwangi sont responsables des affaires publiques; pendant les générations $A^{\prime}, B^{\prime}, C^{\prime}$, cette tâche est dévolue aux anciens maina. Mais, durant une génération mwangi, il $y$ a théoriquement autant d'individus qui sont maina et mwangi. En définitive, et bien que mwangi et maina qualifient les anciens-gouvernants, il est toujours possible de déterminer_avec précision à quelle génération un enfant kikuyu appartiendra : il est, en effet, toujours groupé dans la même moitié que celle de son grand-père paternel et dans la moitié opposée à celle de son père. Cette règle est fixe, mais n'entre en application qu'au moment où l'individu atteint l'âge de $25-30$ ans, c'est-à-dire au moment où il se marie et devient par là éligible au premier degré d'ancienneté. En outre, et quoique la division en moitiés tribales ne touche que les hommes, un homme dont le père est mwangi ne peut épouser qu'une femme dont le père est lui-même mwangi elle est donc groupée comme lui dans la moitié maina (voir fig. 6).

Le terme muirungu (pl. irungu) qui clôt la plupart des listes dressées par les observateurs désigne, ainsi que l'indique C.W. Hobley ${ }^{14}$, la génération montante, qu'elle soit en principe maina ou mwangi. D'une façon plus précise, ce terme s'applique à la génération qui doit passer par les quatre degrés d'ancienneté après la prochaine itwika (rituel de délégation des pouvoirs), c'est-à-dire à la classe des guerriers. Une information donnée par J. Kenyatta permet de déterminer avec précision, eu égard à la position respective des anciens mwangi et maina, quelle moitié connote le terme muirungu. En somme, la division en moitiés tribales jouerait d'une certaine façon

14. Op. cit., 1938, p. 92. 
a) avant litwika (situation en 1925)

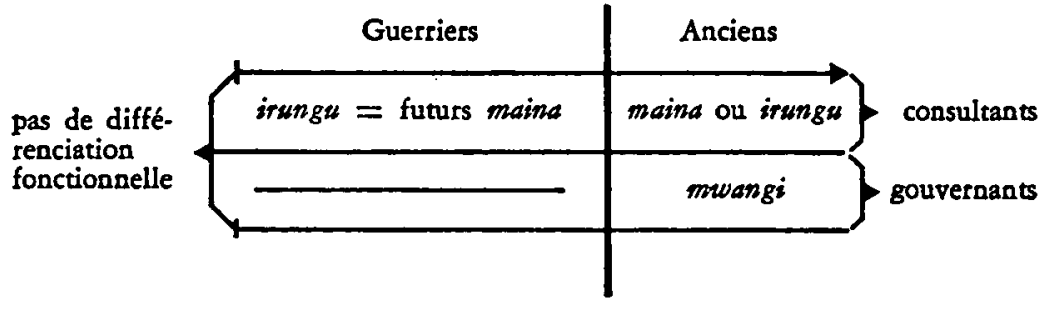

b) après l'inwika (situation théorique après 1925)
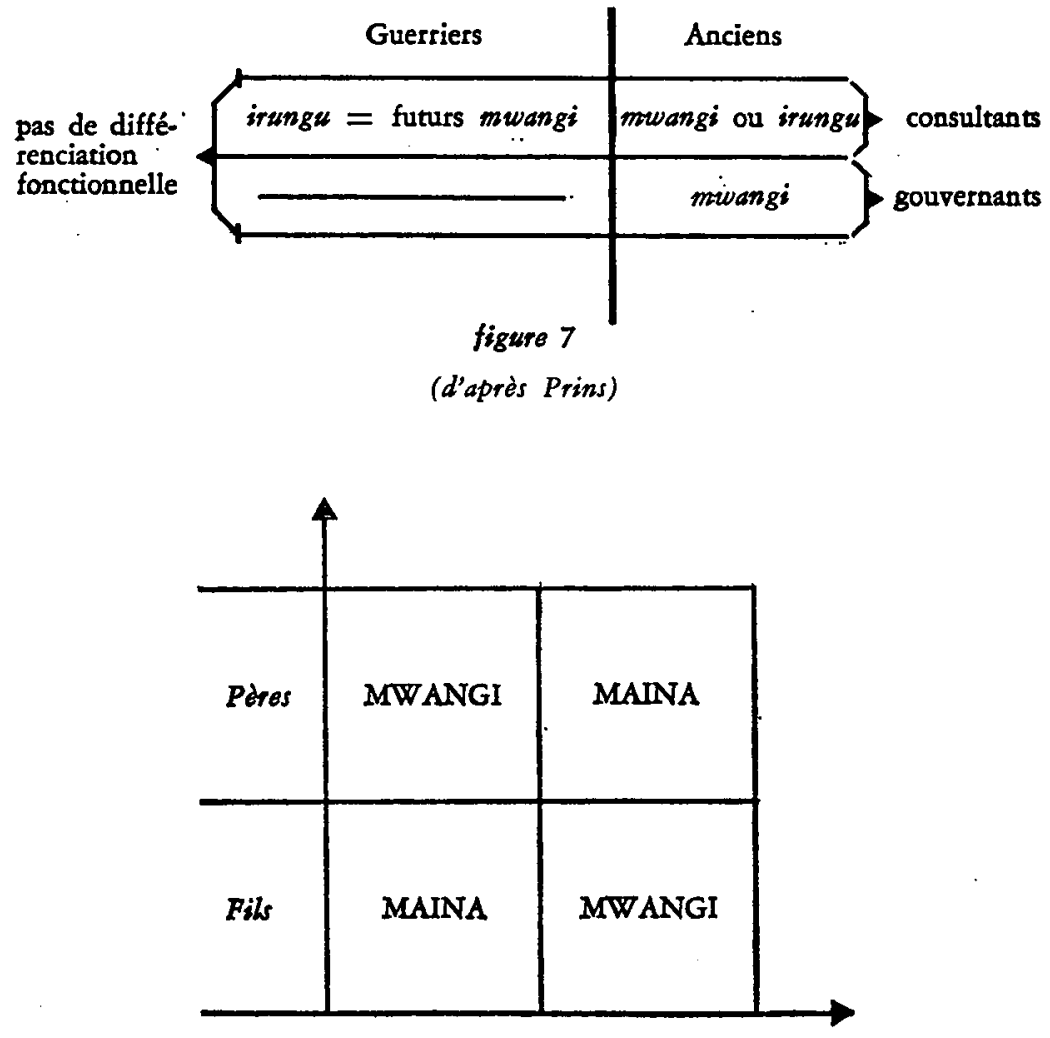

figure 8 
à un niveau sociologique inférieur à celui de l'ancienneté, soit au niveau de la classe des guerriers qui seraient donc déjà différenciés. En effet, dans une remarque, J. Kenyatta pose l'identité sémantique entre maina et irungu : "La génération des irungu ou maina qui devait prendre la relève s'assembla en 1925 et commença les chants et les danses rituels de litwika pour marquer la fin du mandat de la génération mwangi ${ }^{15}$. Dans ce cas précis, irungu désigne les anciens-consultants maina, et les irungu sont des futurs maina. Après l'itwika, la situation s'inverse puisque irungu qualifie toujours la génération montante (voir fig. 7). On peut donc conclure que le terme irungu, dont nous avons dit plus haut qu'il qualifiait la génération montante dans son ensemble, soit la classe des guerriers, désigne en fait toujours et uniquement une partie de cette classe, soit la moitié virtuellement gouvernante (c'est-à-dire celle qui détiendra le pouvoir après l'itwika) qui est maina lorsque la moitié au pouvoir est mwangi, mwangi lorsque celle au pouvoir est maina. Cela introduit donc la partition en moitiés tribales au niveau de la classe des guerriers, sans pour autant introduire une différenciation fonctionnelle : la division reste structurelle. L'autre moitié, non différenciée par un terme propre, est bien entendu de la même moitié que celle au pouvoir. Cette remarque est importante car elle montre que la division en moitiés tribales intervient dès la constitution des classes d'âge, qui voient de ce fait leur homogénéité disparaître derrière une structure généalogique « fictive », \& mimée », puisque les uns deviennent les peres symboliques des autres et participent au pouvoir de leurs pères réels, biologiques (voir fig. 8).

Ainsi que nous l'avons signalé plus haut, il convient de distinguer la classe d'âge de la catégorie d'âge. Cette dernière notion se comprend par référence au degré d'âge, c'est-à-dire qu'on peut la définir comme le groupe social qui occupe un degré d'âge donné. Ce groupe peut être constitué, comme nous allons le voir, par une partie de la classe d'âge ou par plusieurs classes d'âge.

Les degrés d'âge kikuyu sont théoriquement au nombre de cinq : 1) guerrier junior, mumo;2) guerrier senior, anake; 3) \& ancienaspirant $\gg$, karabai ; 4) ancien, mutbamaki mbule; 5) * ancien doyen \$, ukuru. L'accès aux trois degrés supérieurs est réservé aux membres de la moitié gouvernante. Les hommes mûrs de la moitié consultante ne sont pas, quant à eux, sociologiquement différenciés :

15. J. KenYATTA, op. cit., 1967, p. 137. 
ils occupent un \& degré fictif \$ (shadow grade 19 ) qui leur confère le statut général d'ancien. Après l'itwika, la situation se renverse.

1. Mumo et anake : le premier degré auquel accède dans sa totalité la classe d'âge après la circoncision est celui de morika ya mumo, ou catégorie des guerriers juniors. Au bout de six années (en moyenne), le groupe est promu au degré de morika ya anake, ou catégorie des guerriers seniors, qu'il occupe également pendant une période de six années. La cérémonie de passation des pouvoirs (inwika) n'intervient pas au niveau des deux premiers degrés, c'est-àdire que la catégorie des guerriers seniors n'est pas supplantée par celle des guerriers juniors. Il existe au contraire un principe de continuité : le groupe nouvellement admis au degré mumo devra, et cela quoi qu'il arrive au niveau de la structure politique, remplir son contrat d'âge avant de postuler l'entrée dans le degré anake. On peut dire d'une façon générale que, chaque année, des jeunes gens sont initiés avec un décalage théorique de six années au degré mumo et au degré anake. Ainsi, l'effectif de la première classe d'âge demeure en principe constant et chaque membre se trouve au même moment placé dans la même situation sociologique. Il en va, toutefois, autrement dès que les guerriers seniors ont rempli leur contrat d'âge. L'accès au statut d'ancien ( \& degré fictif $>$ d'ancien-consultant, ou premier degré d'ancien-gouvernant, soit le troisième degré dans la structure générale) est en effet caractérisé par des cérémonies qui ne concernent plus le groupe dans sa totalité, c'est-à-dire la classe d'âge, mais chacun de ses membres pris individuellement sur un certain nombre d'années. De fait, les critères retenus pour accéder à ce statut sont d'une part le mariage et d'autre part l'installation du groupe domestique, soit des critères d'ordre \& contingent $>$ et non plus structurel, comme c'est inversement le cas pour les promotions aux degrés mumo et anake. Ce mode de promotion et ses implications sur le groupe social considéré permettent de suggérer que les classes d'áge kikuyu se dissolvent d'elles-mêmes à travers les mariages de leurs membres. La dissolution du groupe annuel est certes une affaire temporaire pour les membres de la moitié consultante, car au bout d'un certain nombre d'années ils se trouvent placés dans une même situation sociale (mariage) et partagent donc le même statut. Du fait

16. A. PRINS, op. cit., 1953, p. 49. 


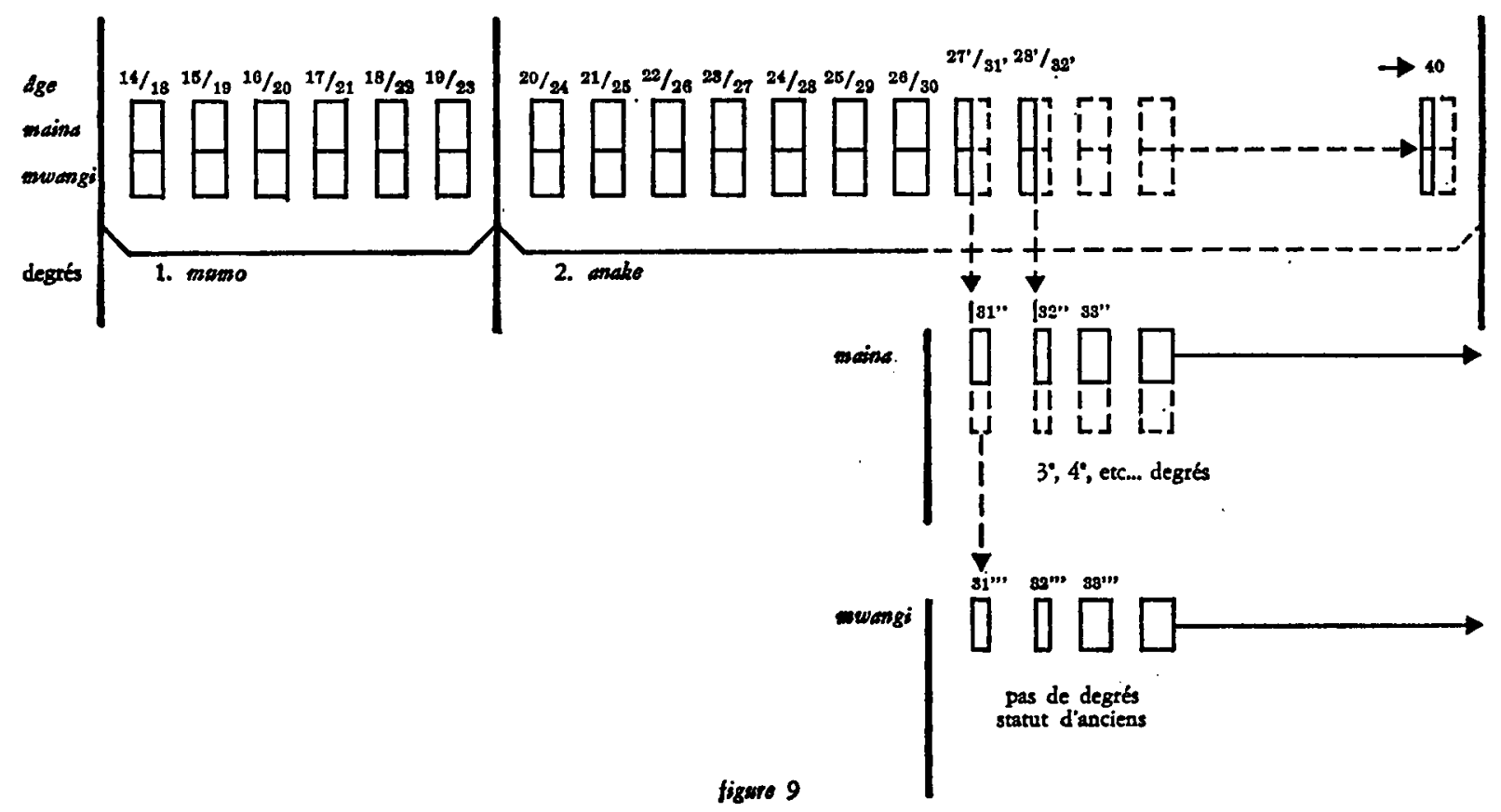


qu'ils ne sont pas structurellement concernés par la stratification en degrés d'âge, ils repèrent leur position dans l'ordre de l'ancienneté selon une semi-hiérarchie » notamment fondée sur la seniorité. Par contre, pour les anciens de la moitié gouvernante, la hiérarchie repose sur les droits et les devoirs liés à chacun des trois degrés. Ainsi et au niveau de l'ancienneté, c'est-à-dire au niveau de l'exercice du pouvoir, la classe d'âge perd de son importance en tant que groupe social. L'accès aux degrés supérieurs dépendant en grande partie de facteurs contingents, on constate que certains individus peuvent se mouvoir plus vite que d'autres dans l'échelle des degrés, et par conséquent que les membres d'une même classe d'âge peuvent être dispersés sur plusieurs degrés. On peut donc avancer que la classe d'âge kikuyu disparaît comme groupe cohérent et homogène au niveau de l'entrée dans l'ancienneté d'abord en raison de la division duelle qui intervient fonctionnellement (maina/mwangi), ensuite du fait du mode de recrutement aux différents degrés. Sur la figure 9, la classe $g$ de degré anake a parmi elle les plus jeunes membres de la catégorie des guerriers seniors; la plus vieille classe d'âge $(m)$ de cette catégorie a une moyenne d'âge de vingt-huit ans, tourefois certains membres peuvent être nettement plus âgés (quarante et au-delà) et sont alors les irui non mariés des classes antérieures. D'une façon générale, les individus de la classe $m$ accèdent à l'ancienneté : ou bien ils appartiennent à la moitié gouvernante, ils entrent alors dans les différents conseils et jouent un rôle prépondérant dans la vie publique (31", 32", 33", etc.), ils sont soumis au régime des degrés d'âge; ou bien ils appartiennent à la moitié consultante et sont alors ce que A. Prins appelle des « anciens sans portefeuille (task-less elders), au tôle purement consultatif, soit les anciens-consultants (31"', 32"', 33"', etc.). Les anciens-gouvernants sont appelés atburi a kiama, qu'on peut traduire par a anciens de conseil $\gg$; les anciensconsultants sont juste appelés atburi (A. Prins).

2. Karabaï : l'accès à ce degré est conditionné par le mariage du candidat et l'établissement de son foyer. Après avoir a payé \ une chèvre au kiama kia itura ${ }^{17}$, le postulant appartient au morika ya karabaĩ, catégorie des "anciens-aspirants 》 (learning elders). Les karabai ont leur propre conseil, le kiama kia kamatimu. Ici, une précision terminologique s'impose car la plupart des auteurs semblent

17. Conseil de village formé par les anciens des quatrième et cinquième degrés. 
s'être mépris sur le sens de kamatimu et l'ont étendu à n'importe quel individu occupant le troisième degré. Le terme karabaī que nous avons employé d'après $\mathrm{A}$. Prins, qui lui-même le tient des informations de Koinange, indique la position qu'occupe un mutburi (ancien) dans l'échelle des degrés (soit le troisième), alors que le terme kamatimu désigne la fonction qu'il peut remplir dans le système des conseils. En d'autres termes, un mutburi peut être karabai sans être pour autant kamatimu. Ce degré est, de l'avis de C. W. Hobley, subdivisé en trois rangs dont l'accès est conditionné par le paiement d'une chèvre. On a ainsi :

1) mutburi ya mburi imwe = "ancien à une chèvre \$

2) mutburi ya mburi irigi $=$ "ancien à deux chèvres \$

3) muthuri ya mburi itatu = "ancien à trois chèvres 》

Ces rangs semblent purement honorifiques. D'une façon générale, la fonction des kamatimu est réduite à celle de messager. Bien qu'ils participent aux assemblées en tant qu'auditeurs, ils ne siègent pas avec les anciens des conseils supérieurs ${ }^{18}$.

3. Athamaki et ukuru : les anciens des quatrième et cinquième degrés sont connus sous le nom générique de athamaki (sing. mutbamaki), qui signifie juge, magistrat ou encore législateur selon les auteurs, ce qui signifie encore qu'ils sont a anciens à part entière \$ (full elders) et peuvent intervenir dans la vie publique par l'intermédiaire des différents conseils auxquels ils sont éligibles compte tenu de leur degré d'âge. Mutbamaki n'indique donc pas un degré (ainsi que C. W. Hobley l'a pensé puisqu'il l'applique aux anciens ukuru), mais connote la position d'un individu ou d'un ensemble d'individus au sein d'un groupe social de quelque échelle soit-il : d'une façon plus précise, il exprime le statut de a leader $\gg$ et peut alors s'appliquer à n'importe quel individu de n'importe quel degré ayant ce statut. C'est ainsi qu'on le trouve au niveau mumo et anake (mutbamaki wa rika) et au niveau karabaï (muthamaki wa bururi). Toutefois, le terme atbamaki, employé seul, semble réservé aux anciens des deux plus hauts degrés.

18. Le terme kamatimu signifie d'ailleurs, selon $C$. W. Hobley (op. cit., 1938), * celui qui siège au loin s. J. Kenyatta (op. cit., 1967, p. 140) fait remarquer que matimu signifie lance et indique par là que le karabaì * est encore un guerrier et qu'il se sert de sa lance puisqu'il n'a pas encore obtenu le bâton de commandement $\gg$ des anciens à part entière. 
L'accès au quatrième degré est déterminé par le fait que le premier enfant mâle est prêt à être circoncis, soit quatorze ans en moyenne après l'entrée du postulant dans le degré karabai. D'après J. Kenyatta ${ }^{10}$, le quatrième degré serait l'avant-dernier, mais les informations fournies par Hobley, Koinange et Prins permettent d'avancer qu'il se subdivise en deux degrés bien distincts. C. W. Hobley désigne les membres appartenant à ces deux degrés par les expressions : 1) atburi cha imburi nne, 2) atburi cha imburi ithano; soit \& anciens à quatre et cinq chèvres \$. A. Prins, d'après les informations orales de Koinange, emploie les expressions : 1) morika ya kiama mbule omwe, 2) morika ya kiama mbule iregi; ou « catégorie des anciens de conseil à une et deux chèvres $\gg$, signifiant ainsi que les anciens des quatrième et cinquième degrés sont des juges, des législateurs, par opposition aux anciens-aspirants du troisième degré qui sont des messagers. Quoi qu'il en soit, les expressions sont structurellement synonymes, mais nous retiendrons plutôt celles de Koinange et de Prins qui marquent d'une façon plus nette la différence de fonction existant entre les anciens karabaï et les anciens atbamaki.

Selon J. Kenyatta, le cinquième degré ukuru n'est atteint que par les hommes les plus âgés de la tribu, c'est-à-dire ceux dont tous les enfants mâles sont circoncis et ceux dont toutes les femmes sont ménopausées. Ils sont alors désignés par le terme atburi a ukuru et forment le « conseil sacrificiel » (kiama kia matutanguru). Nous verrons plus loin les problèmes que soulève l'accès à ce dernier degré.

4. Les conseils: comme l'indique la figure 10 , le kiama kia atbamaki ou a conseil des anciens à part entière > est formé par les anciens des deux derniers degrés (lignes en pointillés), et c'est ce conseil qui détient la majeure partie des pouvoirs. Il est représenté au niveau des différentes unités territoriales selon la portée des problèmes qu'il a à résoudre. Il ne semble pas que ce soit le caractère de l'offense qui détermine la portée territoriale d'un kiama, mais la qualité et la magnitude du groupe qui la commet. Ainsi la « magie noire $\gg$ n'est pas une sorte d'offense, mais un crime qui atteint toute la communauté : son cas est du ressort du kiama kia maturanguru kia qugongo (conseil des ukuru de la crête); le coupable est souvent mis à mort.

Le kiama kia mataathi (conseil de la paix) et le kiama kia matu-

19. Op. cit., 1967, p. 140. 
5. ukeuru

4. atbamaki mbule 1 \& 2

3. karabas

2. Anake

1. mumo

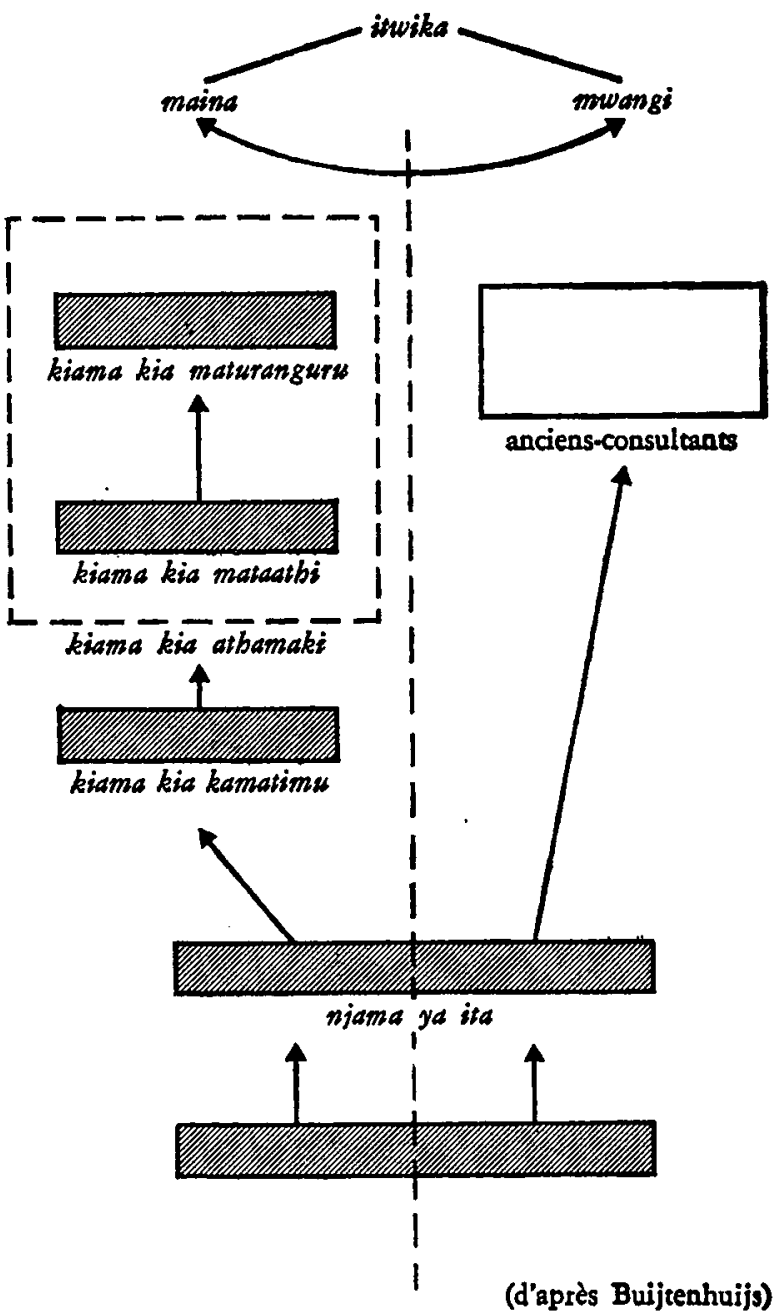

figure 10 
ranguru (conseil sacrificiel) sont ce que $A$. Prins appelle des $<$ conseils fonctionnels $\gg$, c'est-à-dire des institutions propres à certains degrés d'âge (en l'occurrence aux quatrième et cinquième degrés). Ils ne sont pas représentés à l'échelle territoriale, ou du moins, s'ils le sont, c'est $a$ travers les différents conseils territoriaux. Si les anciens des deux degrés supérieurs composent un kiama kia atbamaki à quelque échelle territoriale que ce soit, la stratification par degrés d'âge intervient au niveau de cette institution du fait que les tâches, les droits et les devoirs, en somme le pouvoir des individus qui forment ce corps, diffèrent quant à leur nature et à leur portée selon leur position au sein du système. Ainsi, bien que les anciens des quatrième et cinquième degrés siègent ensemble lors d'une réunion du conseil territorial, ils ne disposent ni d'un statut ni d'un pouvoir identiques au sein du conseil. Ils jugent et décident en fonction du degré auquel ils sont. Chaque cas soumis au conseil territorial, bien qu'il nécessite la réunion de tous les membres, ne peut être entendu que par l'ancien ou le groupe d'anciens à qui le degré confère la compétence pour le juger. En somme, le conseil territorial représente plutôt qu’il ne détient la totalité des pouvoirs.

Les anciens qui appartiennent au kiama kia mataathi sont théoriquement investis des pouvoirs administratifs et judiciaires. Toutefois, une certaine différenciation de fonctions intervient au niveau de cette institution : en théorie, la fonction des anciens du quatrième degré est d'entendre les cas, celle du « quatrième bis » (les athamaki mbule iregi de A. Prins) étant de les juger. Ce conseil décide dans son ensemble des sacrifices à faire à Ngä̈ (le * dieu > des Kikuyu), mais seuls les anciens ukuru peuvent sacrifier et approcher l'arbre sacré (mugumu). Ces derniers, qui forment le kiama kia maturanguru, ont essentiellement des pouvoirs rituels, cérémoniels et religieux. Ils décident des sacrifices et prières pour la pluie, pour les ancêtres, etc., conduisent les cérémonies qui marquent le calendrier agricole ${ }^{20}$, exécutent rites de purification et sacrifices. Dans certains cas, et notamment pour la date de la circoncision et de l'itwika, ainsi que pour l'admission à un degré supérieur d'ancienneté, les décisions sont prises par le kiama kia maturanguru.

Au niveau de la réalité, les différents conseils ne comportent pas un nombre fixe et constant d'anciens. Il n'y a pas non plus de conseils permanents ni de lieux précis de réunion : les conseils se

20. Cf. J. JAMIN, Contacts et antagonismes culturels..., op. cit., 1973, p. 94. 
forment « à l'occasion *. De même, les anciens intervenant dans un cas peuvent être remplacés par d'autres dans un autre, selon leur niveau de compétence. Les différents conseils apparaissent donc comme des réalisations indéfinies des degrés d'âge toujours définis. Tout se passe comme si une catégorie d'âge déléguait l'exercice du pouvoir dont elle est théoriquement investie à un certain nombre de ses membres qui, du fait de leurs capacités, de leur statur, et à ce moment, formaient un certain conseil (A. Prins).

Au terme de ce bref examen, il apparaît que les conditions à remplir pour se mouvoir à l'intérieur des degrés d'ancienneté sont des conditions d'âge, de fortune, de valeur personnelle et de statut social et généalogique des enfants. Cela est vrai pour l'ensemble et notamment pour l'accès aux troisième et quatrième degrés, mais le problème se complique dès que l'on envisage l'accès au cinquième degré, car, ainsi que le fait remarquer J. Middleton ", l'âge, la fortune et la sagesse ne semblent pas des titres suffisants pour être admis à ce degré. En effet, seuls les chefs de patrilignage (mbari, appelés aramati) peuvent le postuler. En somme, le critère devient ici le statut généalogique. Cela n'a rien d'étonnant si l'on sait ${ }^{23}$ que le groupe qui offre les sacrifices aux dieux et aux ancêtres est de l'ordre du mbari; il semble alors \& logique \$ que les anciens sacrificateurs qui servent de médiateurs entre dieu et les hommes, entre les morts et les vivants, se recrutent à la tête des différents mbari, c'est-à-dire au statut généalogique le plus haut. On peut donc dire que les anciens ukuru représentent les différents mbari et qu'il existe une relation statistique entre le nombre de mbari et l'effectif de la catégorie ukuru. C. W. Hobley remarque que les anciens ukuru étaient pour la plupart des vieillards, mais qu'il n'était pas rare de trouver parmi eux des hommes plus jeunes. Ce qu'il explique en disant que les vieux recrutaient par cooptation des hommes plus jeunes ayant une réputation de sagesse. Cette explication n'est cependant pas satisfaisante, d'abord parce que le statut généaologique dans ce cas est peu manipulable, ensuite parce que les ukuru jouissent d'attributs \& spirituels \ qui leur sont personnels ${ }^{23}$. Ce qui semble plus probant, c'est la remarque que C. W. Hobley fait plus loin :

21. Les Kikouyous at les Kambas du Kenya.., op. cit., 1954, p. 58.

22. J. JAMIN, Contacts et antagonismes culturels..., op. cit., 1973, p. 57.

23. Cf. J. MIDDLBTON, op. cit., 1954, p. 59. Seuls les anciens ukuru sont enterrés, les défunts des autres catégories sont jetés dans la brousse où ils servent de pâture aux hyènes. 
« La famille d'un muthamaki (ukuru) doit toujours être représentée dans ce grade. S'il arrive qu'un ukuru meure et ne laisse aucune autre relation qu'un jeune homme, on élira son fils ou son frère à sa place, même s'il est encore jeune ${ }^{24}$. \En outre, et compte tenu d'une part des conditions d'accès au degré ukuru (statut généalogique), et d'autre part de la dynamique sociale (segmentation et fondation de nouveaux patrilignages) ${ }^{25}$, il n'est pas surprenant de constater que des hommes jeunes soient présents dans cette catégorie. Cela ne répond pas tourefois aux conditions d'accès décrites par J. Kenyatta, reprises par A. Prins : à savoir la circoncision de tous les enfants et la ménopause des épouses. Il est possible que J. Kenyatta ait voulu exprimer par là une norme, auquel cas on reprochera à A. Prins de l'avoir prise pour la réalité et d'en avoir déduit la présence nécessaire des atbamaki mbule 2 au conseil 5 (voir fig. 10), composé « des hommes les plus vieux de la communauté », afin de suppléer aux disparitions éventuelles des ukuru. Si un tel mécanisme joue, il contredit les observations de $C$. W. Hobley de même que les conclusions de J. Middleton, qui laissent entendre que le statut d'ancien ukuru est prescrit et dépend de la position qu'occupe l'individu au sein de la structure lignagère. On a donc tout lieu de penser que le degré ukuru se situe en dehors du système des classes d'âge. Dans cette perspective, bien qu'étant nécessairement groupés dans une moitié tribale, les anciens ukuru ne devraient pas être atteints par le principe d'alternance des générations, qui ne concernerait alors que les anciens des degrés inférieurs. Il serait en effet surprenant qu'un individu dont les fonctions sont chargées d'une valeur mystique, dont le pouvoir est d'ordre quasi surnaturel, dont la position en fait le médiateur entre l'ordre religieux et l'ordre social, soit soudain démis de ses fonctions et retourne au \& profane \$.

La tendance égalitaire et démocratique du système des degrés et des classes d'âge kikuyu, théoriquement organisé sur la base de la compétence et de la valeur personnelle - tendance soutenue et souvent citée en exemple par quelques auteurs ${ }^{26}$-, est donc pour

24. C. W. НовLEY, op. cit., 1938, p. 212.

25. Cf. J. Jamin, Contacts et antagonismes culturels..., op. cit., 1973, p. 88 et $s$.

26. J. KenYaTTA, op. cit., 1967, p. 131-158; L. MaIR, Primitive Government, Penguin Books, Londres, p. 98-106; J. MIDDLEToN, op. cit., 1954, p. 51.61 ; W. E. MŨHLMANN, Messianismes révolutionnaires..., op. cit., 1968, p. 91. 
une large part contestable, voire illusoire. Le parasitage de l'ordre lignager n'est pas aussi évident qu'il paraît :

1. Le système mwangi/maina introduit, dès la constitution des classes d'âge, une division selon un modèle généalogique qui détermine les conditions d'accès et les écarts au pouvoir. En raison de la transformation historique des générations en moitiés tribales ${ }^{27}$, une relation symbolique père/fils intervient au niveau de la classe d'âge et recoupe une position de pouvoir puisque les pères symboliques sont de la même moitié que les pères biologiques au pouvoir. En outre, comme le fait justement remarquer R. Buijtenhuijs, \& un jeune homme au début de sa carrière pouvait être favorisé par sa position généalogique au sein d'un mbari (patrilignage), dans la mesure où la famille étendue constituait pour lui un premier champ d'action où il pouvait faire valoir ses aptitudes pour les affaires publiques. Le muboi (locataire des terres d'un mbari) qui ne vivait pas en mbari n'avait au départ qu'un champ d'action restreint; il courait le risque de ne pouvoir " décoller " et de rester ainsi pendant toute sa vie en retard sur ses compagnons d'âge nés dans des familles de propriétaires ${ }^{28} \gg$.

2. Les conditions d'accès au degré ukuru réintroduisent d'une façon marquante et impérative la problématique lignagère. Bien que théoriquement cantonnés dans des fonctions symboliques et religieuses, les anciens ukuru cumulent, de par leur position (chefs de patrilignage), un pouvoir foncier (distribution et répartition des terres) et un \& pouvoir matrimonial > (distribution des femmes et paiement de la dot aux fils et aux frères cadets) qui limitent et endiguent de fait la mobilité sociale à l'intérieur des degrés d'âge, puisque l'accès au troisième degré karabaĩ nécessite le mariage et la possession d'une terre, puisque les promotions ultérieures dépendent de leur avis. Les prestations en chèvres qui marquent les franchissements d'échelons permettent aux anciens ukuru qui les reçoivent d'accumuler et de capitaliser du bétail : « monnaie » privilégiée dans les transactions foncières et les prestations matrimoniales, il leur confère le pouvoir économique. Ils interviennent donc directement, par la conversion du capital bétail en capital foncier, dans l'expansion terri-

27. Thèse de R. BujJTenturjs, Le Mouvement Mau-Mau..., op. cit., 1969, p. 31 .

28. Ibid., p. 39. 
toriale et dans le processus de colonisation des terres neuves (notamment dans le Kiambu à la fin du $\mathrm{XIX}^{\circ}$ siècle). De plus, le choix du moment de l'itwika qui est de leur ressort autorise le contrôle sur le jeu politique à l'intérieur de la tribu (l'itwika intéressait en effet l'ensemble des pays kikuyu).

Bien que ce système soit, selon la perspective esquissée par J. Kenyatta et reprise par ailleurs, sur un plan plus théorique, par Eisenstadt et $\mathrm{D}$. Paulme, le résultat d'une détérioration progressive de l'organisation clanique, il ne se situe pas à côté et n'opère pas contre l'ordre lignager : il en est une expression et une extension sociologiques. Les classes d'âge kikuyu stricto sensu, dont la fonction semble, de l'avis des observateurs, essentiellement militaire, sont en tout cas inféodées au système lignager qui réapparaît « en bout de course \$ au niveau des ukuru et contribuent de ce fait à en garantir l'ordre et à en assurer l'extension, le développement et la reproduction. Le langage démocratique des classes d'âge camoufle, tait en somme la hiérarchie lignagère fondée sur le principe généalogique, sans pour autant l'évacuer complètement puisque c'est lui qui finalement organise et ordonne les degrés. $\mathrm{La}$ valeur et la compétence personnelles jouent certes à l'intérieur d'une même classe pour la prise et l'exercice du pouvoir au sein de celle-ci, mais sont par contre délaissées, dès que l'on envisage le fonctionnement global du système, au profit de la place généalogique qui conditionne et détermine alors le passage d'une catégorie à l'autre et le pouvoir de l'une sur l'autre. Dans cette perspective, les rapports de parenté deviennent à la limite des « rapports de classe 》, dans la mesure où la position généalogique est symboliquement étendue à chaque individu placé dans une a même situation de classe »(cas des mwangi/maina). La multiplication des écarts et des différences, notamment repérable dans le jeu des rangs et des degrés, renforce l'ordre lignager en démultipliant les prises de son pouvoir et en élargissant spatialement son champ d'application sociale.

La raison et le sens de l'ambiguïté, voire de la duplicité, sociologique des classes d'âge - dont le système kikuyu offre un exemple pertinent ${ }^{20}$ - peuvent être éclairés par l'examen des rituels qui accompagnent leur constitution, et que l’on qualifie généralement et peut-être abusivement d' initiation tribale $\gg$. C'est sans doute ici,

29. M. Augé a également insisté sur ce point, dans sa thèse d'Etat (op. cit., 1975), à propos des classes d'âge en milieu lagunaire (Côte-d'Ivoire). 
dans cet espace et ce temps de passage, de formation et de transformation de l" « adolescent 》 en " homme », en « adulte », que transparaît et que se repère avec force ce que j'ai appelé plus haut * le paradoxe initiatique \$. L'initiation est, pour reprendre la terminologie de $\mathrm{R}$. Bernstein ${ }^{30}$, à la fois * consensuelle » dans sa fonction d'unification, d'inculcation et de normalisation des valeurs culturelles, de production d'un état plus que d'un statut; et * différenciatrice * dans son mécanisme, certes peu évident, en tout cas peu décrit ou analysé, de mise en place sociologique selon l'âge, le sexe bien sûr, l'appartenance sociale et la position généalogique. Chacun est mis dans une même situation, mais n'a pas les mêmes chances... ne serait-ce que par le jeu des amendes et taxes rituelles qui accompagnent souvent l'initiation et qui sont généralement supportées par le groupe familial : sa dimension, sa richesse, sa solidarité, son autorité en conditionnent l'ajustement et le paiement et, en conséquence, interviennent directement dans $l$ ' « avancement initiatique » du jeune homme. J. Kenyatta insiste justement sur cette pesanteur du lignage dans les affaires initiatiques et dans les promotions à l'intérieur du système des degrés d'âge : « Ni garçons ni filles, écrit-il, ne peuvent avancer d'un degré d'âge à un autre sans le consentement et l'assistance des parents. Aucun désir, aucune ambition du jeune homme ne peuvent être satisfaits s'il y a une opposition familiale. Sans l'accord des parents, le garçon ne peut être circoncis; sans eux, il ne peut se marier car il ne possède rien en propre, il ne peut assurer les cadeaux et les échanges auxquels les familles sont seules habilitées à procéder. Sans la permission de son père, il ne peut se rendre à un festin de viandes, ni à une réunion où l'on boit de la bière; avant d'en avoir sa part, il doit d'ailleurs en apporter à ses pères et mères ainsi qu'à ses oncles et tantes ${ }^{\text {gI. }}$.

Chez les Sénoufo Kiembara, le recrutement au poro typologo est assez lourd de présupposés généalogiques et économiques. Le tyologo, d'une durée de sept ans, représente la phase suprême du système initiatique, la seule actuellement en vigueur et la mieux « défendue et protégée $\$$. Le tyologo s'apparente à une classe d'âge de type

30. Dans J. HuXLex, Le Comportement rituel chez l'bomme ot l'animal, Gallimard, Paris, 1971, p. 276-288.

31. J. KenYatTA, op. cit., 1967 , p. 90.

32. Bien que des cultes messianiques, tels celui de Massa (1952) et Moussa (1962), en ébranlent périodiquement la structure. La fréquence de ces prophétismes serait, selon G. Bochet (art. cité, 1965), une réponse probable 
classique, par les liens de coopération et de pseudo-parenté qui unissent ses membres (ils s'appellent nortiarbélé, c'est-à-dire \& frères de promotion », ou encore téfalanionbélé, soit 《 compagnons de culture $\gg)$, sans pour autant évacuer la structure généalogique. L'interdiction rituelle faite à deux frères utérins d'être nortiarbélé dans un même tyologo implique théoriquement un bandicap de sept années pour le cadet, quelle que soit la différence d'âge avec l'aîné ${ }^{33}$. Handicap qui a des répercussions sociales (situation matrimoniale : c'était en principe au cours des deux dernières années du tyologo que le * jeune > Sénoufo recevait une femme), économiques (cession ou agrandissement de la parcelle personnelle lors du mariage) et culturelles (position vis-à-vis du dispositif symbolique mis en scène lors des funérailles et pris en charge par les initiés), ces répercussions accusant la distance et le rapport de dépendance entre les aînés et les cadets (ces derniers devant, une fois promus, travailler pour leurs aînés, « sortis 》, de ce fait assimilés aux « vieux \$ qui distribuent et coordonnent la force de travail). Ainsi, et au lieu que la promotion repose sur lâge biologique (cas des systèmes dits linéaires), elle dépend de l'âge social : une promotion donnée groupe théoriquement les cadets sociaux d'une promotion antérieure, et cela explique les écarts d'âge que l'on peut observer à l'intérieur d'une même promotion.

La pression des sociétés locales de développement qui tendent à avancẹ l'âge de la « maturité sociale \$ (attribution de parcelles à des volontaires) et de la responsabilité économique, les effractions périodiques de l'univers symbolique par des cultes prophétiques d'inspiration islamique, le développement des moyens de communication qui fissurent les terroirs et territoires traditionnels, le mécontentement des jeunes générations devant la rigidité d'un système qui, par le jeu des amendes et taxes rituelles, du travail obligatoire, quasi forcé, pour les vieux, mécontentement qui se manifeste par la fuite « à la ville \$ ou en « basse côte - - tous ces facteurs ont finalement nécessité des ajustements progressifs qui ont abouti en certains endroits,

au carcan idéologique qui maintient les Sénoufo dans un climat d'interdits, d'agressions, de peur, voire de terreur, et qui se manifeste notamment lors de l'initiation où le jeu des effets et des procédés d'agression accuse la fonction disciplinaire et normalisatrice de l'institution.

33. La transgression de cet interdit est interprétée en termes de cannibalisme : * Si deux frères de même mère font le poro dans le même bois sacré, le plus jeune mangera le plus vieux. 
notamment en zone dense (région de Korhogo), à disperser géographiquement ceux qui étaient généalogiquement distancés. S'inspirant d'un modèle traditionnel en cas de gémellité, les aînés et les cadets peuvent être répartis, dans un même temps, dans des bois sacrés différents. Ce changement, tout juste amorcé, permet aux cadets d'acquérir un statut social équivalent à celui de leurs aînés, mais les coupe pour des périodes certes limitées mais répétées de leur milieu social d'origine et les éloigne tout autant des prises de décision, puisqu'ils se trouvent fréquemment mis à l'écart des activités sociales et rituelles de leur village par leur assujettissement à celles de leur village d'accueil. Le choix du bois sacré revient théoriquement au chef de lignage, mais je n'ai trouvé aucune règle précise et clairement exprimée : le cadet peut, par le jeu de ses relations, décider de l'endroit où il * fera le poro ». Cela a pour conséquence de déconnecter le critère de l'âge social par l'élimination plus ou moins forcée (actions administratives et économiques, voire scolaires) du handicap généalogique et de vider de leur contenu idéologique et rituel les cycles initiatiques précédents dont le tyologo devait être l'aboutissement (cf. infra).

Cette situation nouvelle, peut-être en voie de consolidation, pourrait expliquer l'extension et le développement de ce « petit mariage \$, à l'origine proprement nafara, appelé kékourougou (cf. supra, chap. 2) dans d'autres ethnies, notamment les Kiembara, et que L. Roussel avait constaté pour d'autres raisons : « Aux questions posées pour tenter d'éclairer le sens et l'évolution actuelle sur ce point [le développement du mariage kékourougou], les réponses sont multiples et souvent peu convaincantes : "Aujourd'hui nous ne vendons plus nos femmes, elles sont libres." Certes, mais cette liberté aurait pu aussi bien se traduire par la diffusion d'un type de mariage fondé sur un contrat entre les conjoints, ce qui paraît encore très éloigné de la réalité. Une autre explication est souvent avancée : " Les vieux ne donnent plus de femmes parce que les jeunes refusent de travailler pour eux comme autrefois." Ce thème contient sans doute une part de sincérité plus grande que le précédent. Il y a certainement, pour celui qui détient la puissance paternelle sur la femme, plus d'intérêt à la marier sous le régime du kékourougou que sous celui du tyéporogo ${ }^{34}:$ il s'assure

34. Il s'agit, chez les Nafara, d'une forme d'union matrimoniale symétrique et inverse du kb́kourougou, avec cession théoriquement définitive de la femme et de la descendance au lignage du mari, contre une forte compensation matrimoniale. Chez les Kiembara, ce type de mariage n'existe pas, tyéporogo désigne pour eux la mise en gage de la nièce utérine. 
ainsi la dévolution des enfants issus de cette union, en même temps qu'il rend permanentes les prestations annuelles de travail de son "gendre ": alors qu'autrement celles-ci s'arrêtaient pratiquement après la cession de l'épouse. Dans une période où les motivations économiques deviennent de plus en plus déterminantes, il est difficile de refuser à cette thèse une certaine vraisemblance. Elle est d'autant plus plausible que les vieux qui refusent de laisser partir les "fiancées" de leur concession ont obtenu, eux, de faire venir leur femme dans la leur. Tout se passe, en effet, actuellement comme si pendant une période assez longue, souvent plus de dix ans, la femme restait fiancée et demeurait dans la concession maternelle. Par la suite, et après arrangement entre l'époux et la famille, elle peut rejoindre le village du mari. Il va de soi que cet arrangement appelle quelques avantages matériels pour le père, l'oncle ou le frère de la femme. Les vieux, de cette manière, voient leurs bénéfices augmenter, sans être euxmêmes sujets aux inconvénients du système. Il s'agirait en somme d'une tentative des vieux pour retrouver, par ce biais, une partie des privilèges que "l'évolution" leur retire peu à peu ${ }^{35}$. ,

Le fait que le cadet \& fasse le poro » dans un autre village lui permet d'obtenir, en échange des prestations en travail fournies dans le cadre du poro pour les anciens du village, une femme du village d'accueil. Mais sa dépendance à l'égard de sa famille d'origine l'oblige soit à résider ${ }^{36}$, soit à travailler quelques jours par semaine dans la concession et sur les champs de celle-ci. Aussi n'est-ce pas fortuit de constater, de la même façon que $L$. Roussel le fit en 1963, que l'adoption et l'extension du mariage de type kékourougou se rencontrent principalement dans les jeunes classes kiembara, précisément chez les cadets sociaux. Ia \& double résidence \& impliquée par la transformation du mode de recrutement au poro et par le mariage kékourougou, qui peut en être une conséquence, finit par dénouer les « liens qui doivent exister entre le paysan et sa terre \ et par favoriser, d'une certaine manière, le « déracinement » des jeunes Kiembara et leur mobilité, en dédoublant leur cadre de référence et en créant un espace qui leur permet de mieux se mouvoir et de mieux s'affranchir des « vieux » de leur village d'origine. Si le développement du kékourougou paraît procéder, comme le remarque

35. L. ROUSSEL, op. cit., 1965, p. 44.

36. Il est, ainsi que nous l'avons vu plus haut, théoriquement assujetti en tant que cadet à l'autorité de l'oncle maternel. 
L. Roussel, d'une attitude défensive, il provoque en même temps dans la structure villageoise, une brèche qui ébranle la \& gérontocratie \mentanément et apparemment avantagée par le système $^{37}$ : la dispersion géographique de la force de travail de même que celle des structures d'autorité ne font qu'accroître les tensions entre les aînés et les cadets et favorisent presque la fuite de ces derniers ou bien leur refuge dans l'espace social ainsi crée. Il y a ici mise en place d'un système qui, et d'une façon paradoxale, en poussant l'idéologie matrilinéaire jusqu'à ses derniers retranchements logiques - puisque la \& sœur > devient l' \& épouse sociale \ du * frère $\geqslant$ par sa résidence et la destination de sa descendance, acquise au matrilignage - rompt l'équilibre villageois en provoquant la circulation des hommes et en variant leur mode d'inscription au terroir. Situation pour le moins traumatisante pour une société paysanne où l'espace villageois constituait le seul cadre de référence. De même, la circulation et la superposition des valeurs et des codes provoquées par la « double résidence » ou la « double appartenance \$ des cadets sociaux, tant sociale (kékourougou par exemple) que rituelle (bois sacrés, funérailles et sacrifices), désamorcent la puissance et la signification de l'appareil idéologique traditionnel que soulignait le cadre restreint mais totalisant de la communauté villageoise autocentrée, quasi autarcique, et autorisent une distanciation culturelle et symbolique qui peut parfois aller jusquà la remise en question.

Ces différents ajustements et transformations ont abouti à un abaissement notoire de l'âge d' * entrée » (et bien entendu de « sortie s) au poro tyologo. M. Prouteaux ${ }^{3 s}$ évaluait l'âge d'entrée entre vingt-huit et trente-quatre ans. Actuellement, il serait de vingt à vingt-quatre ans. Compte tenu des fonctions économiques du poro tyologo (travaux sur les champs collectifs et sur ceux des dignitaires du poro), cet abaissement \& diminue d'autant la somme des contributions fournies à la collectivité par les jeunes \$ et alimente les tensions entre les jeunes et les vieux, « ces derniers s'estimant frustrés, puisque les prestations qu'ils ont versées dans leur jeunesse sous forme de travail ou d'amendes ne sont compensées que partiellement par la

37. Cela pourrait, dans une certaine mesure, rendre compte des mouvements sociaux et idéologiques et de l'habitat dense mais dispersé chez les Nafara (par opposition aux Kiembara qui ont un habitat concentre) qui pratiquent couramment ce type de mariage.

38. * Premier coup d'œil sur la religion séné \$, art. cité, 1921, p. 238. 
nouvelle génération. Ils se présentent volontiers comme des victimes dont les créances ne sont pas honorées ${ }^{39} \gg$.

\section{Le silence des savoirs}

Bien que souvent notées dans la littérature ethnologique, la dimension socio-économique de l'initiation, son articulation à la structure sociale, en un mot sa fonction de reproduction sociale ont été non moins souvent négligées ou évacuées au profit de ses fonctions pédagogiques, symboliques et culturelles. L'accent est généralement mis sur la « production de l'homme $\gg$ et sur la « reproduction de la culture $\gg$ (perçue comme « propriété indivise de toute la société $\left.{ }^{40}\right)$, par l'étude des rites qui actualisent et incorporent normes, valeurs et symboles " ${ }^{4}$. Ce qui est d'emblée donné comme appareil de socialisation se voit du même coup, et dans un même mouvement, neutralisé par une approche et une démarche marquées de présupposés philosophiques, psychologiques, voire psychanalytiques ", qui tendent à privilégier les mécanismes de formation et d'information de l'individu sur ceux de distribution et de répartition des individus. Le discours tenu à ce propos sur le mode du singulier pluriel (on parle de quelques-uns pour tous) traduit assez bien cette attitude, tant du côté de l'observateur que de celui des observés, et dissimule pour une part les rapports de forces qui \& sont au fondement de sa force $\gg$ : celle de sélection, de légitimation et d'inculcation. Il serait fastidieux de dresser ici un inventaire des thèses, théories et interprétations en présence, on peut toutefois dégager un certain nombre de thèmes et de directions qui préciséront notre propos.

Un courant de l'ethnologie contemporaine, influencé dans le vocabulaire, sinon dans la conception, par les auteurs de L'Anti$\mathscr{C}$ dipe, considère le fait initiatique comme un processus de marquage,

39. L. Roussel, op. cit., 1965, p. 55.

40. Cf., pour critique, P. BourdiEu et J.-C. PASSERon, La Reproduction..., op. cit., 1970, p. 25 ; et, pour illustration de cette démarche, l'ethnoscience américaine.

41. Cf. notamment les travaux de l'école de Griaule et ceux de D. ZaHAN, Religion, spiritualité et pensées africaines, op. cit., 1970.

42. Cf. les études de G. Roheim, A. Kardiner, et plus récemment celles de B. BeTIBLHEM, Les Blessures symboliques, op. cit., 1971. 
un mécanisme d'inscription, ou encore comme une machine imprimante : il serait à la fois, comme pourrait l'écrire M. Foucault (1975), marque (force du pouvoir), signe (corps social) et trace (appareil rituel). Le corps réel ou symbolique est ici utilisé comme parchemin où viennent s'inscrire les codes et les valeurs culturelles par une série d'épreuves, de « tortures $\gg$, de marques, de traces et de coupures. $P$. Clastres, revendiquant cette filiation théorique, souligne assez fortement cette prise en compte et en charge des corps par la « loi primitive > : Presque toujours, écrit-il, le rite iniriatique passe par la prise en compte du corps des initiés. C'est, immédiatement, le corps que la société désigne comme espace seul propice à porter le signe d'un temps, la trace d'un passage, l'inauguration d'un destin ${ }^{48}$. S'il est vrai, comme il le dit plus loin ${ }^{44}$, qu'un homme initié est un homme marqué, on peut s'interroger sur le sens, la fonction de cette marque, de cette coupure, quelle soit réelle ou imaginaire.

S. Moscovici ss, interprétant les faits que rapporte R. Jaulin dans La Mort Sara (1971) et reprenant une perspective déjà esquissée par G. Bateson (1971), envisage l'initiation comme lieu et temps de transfert des enfants mâles du groupe des femmes au groupe des hommes. La mise à l'écart des femmes, le symbolisme souvent explicite de la mort et de la renaissance dans certaines phases du rite, la dureté, voire quelquefois la « cruauté », des épreuves physiques qui, selon Moscovici, tendraient à exorciser la part de féminin que renferme le corps masculin - tous ces faits, souvent observés, paraissent accréditer cette thèse. Ils évoquent en tout cas \& l'arrachement du jeune garçon à la situation infantile, à l'ambiance maternelle et féminine ${ }^{46}$. Ce symbolisme, sur lequel ont volontiers insisté les psychanalystes, s'accorde pourtant assez mal avec la pratique sociale de certaines sociétés qui, tout en manipulant par le biais de l'initiation un formidable appareil symbolique et rituel, n'opèrent pas moins le « sevrage sociologique \avant, d'une façon certes peu spectaculaire mais tout aussi significarive. Les Sénoufo, par un jeu de cycles de sept ans chacun, échelonnaient traditionnellement l'initiation sur une période de vingt et un ans. Il semble que les deux premiers cycles soient aujourd'hui tombés en désuétude.

43. P. Clastres, op. cit., 1974, p. 154.

44. Ibid., p. 157.

45. Le Société contre nature, op. cit., 1972, p. 287-308.

46. J. Cazeneuve, Les Rites et la Condition bumaine, op. cit., 1958, p. 327. 
Le premier cycle, porowo ou « poro noir \$; coïncide sensiblement avec la période prénubile et se divise en deux degrés :

- gbora, ou l' « apprentissage 》, intéresse les enfants de sept à dix ans, qui apprennent les chants, les danses sous la direction de leurs aînés et qui, dans un passé récent, avaient pour tâche d'assurer le gardiennage (troupeaux, champs, protection des cultures, etc.);

- kamuru (de dix à quinze ans), ou « acquisition de la houe , (kamagé), représente la phase d'apprentissage des techniques culturales sous la direction du père ou de l'oncle maternel.

Sur les deux degrés suivants qui devaient autrefois constituer un cycle, le diégi (15-18 ans) et le fédjen (19-22 ans), probablement le kwor et le kwonro de $\mathrm{B}$. Holas ${ }^{47}$ - qui désignent actuellement la période précédant le cycle tyologo (21-22 ans) - je n'ai pu obtenir d'informations valables, et ils sembleraient, du moins chez les Sénoufo Kiembara, être abandonnés. L' « arrachement » à la mère intervenait donc assez tôt - du moins si l'on tient compte de l'initiation proprement dite, soit le tyologo —, lors du kamuru, où l'enfant mâle passait sous le contrôle économique et culturel des hommes de son lignage.

Chez les Kikuyu, la cérémonie de la seconde naissance, qui a lieu vers 7-10 ans, donc quelques années avant l'initiation, marque la sortie de l'enfance. Tant que l'individu n'a pas subi ce rite, il ne peut ni être circoncis, donc initié, ni hériter de biens, ni participer aux cérémonies religieuses ${ }^{48}$. La mère \& prend le garçon entre ses jambes. On la relie à lui par un boyau de mouton. Elle gémit comme si elle accouchait et le garçon vagit comme un nouveau-né. On coupe alors le boyau de mouton, qui représente évidemment le cordon ombilical. La cérémonie consiste donc à mimer une nouvelle naissance ${ }^{9} \$$.

D'une façon à la limite plus théorique, les travaux de J. RabainZempléni sur l'enfant wolof permettent de repenser la question de la socialisation et de $\mathrm{l}^{\prime}$ \& endoculturation $\gg$ (Herskovits), trop souvent perçue et repérée lors de la « puberté sociologique \$ ( $R$. Benedict), soit au moment de l'initiation. L'apprentissage et l'utilisation de la parole, d'emblée, place l'enfant dans une grammaire sociale qui exprime des coupures, des écarts et des différences : " L'enfant, écrit-elle, apprend un lexique des relations sociales dont il doit

47. Los Sénoufo..., op. cit., 1966, p. 150.

48. Cf. W. S. et K. Rourladge, Witb a Prebistoric People..., op. cit., 1910 , p. 151 .

49. J. CAZENEUVB, op. cit., 1958, p. 337. 
faire usage sur le plan du comportement, mais dont il ne peut faire état librement sur le plan du discours. Le code social qui définit la nature, le destinataire approprié du message et le moment opportun pour le prononcer limite et endigue les possibilités d'expression de l'enfant. Il ne suffit pas de pouvoir dire, il faut encore avoir le droit de dire. Tout se passe comme si les adultes désiraient que l'enfant manifeste une certaine curiosité, une compréhension des relations sociales, qu'il acquiert un certain savoir tout en limitant son droit d'usage. Ce savoir doit être rendu pertinent par l'apprentissage d'une temporisation ou d'une spatialisation du discours. L'enfant devra circonscrire le lieu social pertinent pour pouvoir parler et, quel que soit ce lieu, savoir reconnaître le moment opportun pour prendre la parole. Il apprendra à différer et à censurer. L'accession au pouvoir-dire se fera avec l'accession à une autre catégorie sociale, une autre position hiérarchique. Ainsi l'initiation, où le jeune homme ne recevra pas tant un enseignement spécifique qu'il ne sera mis en situation sociale de dire ce qu'il savait déjà ${ }^{\text {so }}$. > Cette dernière remarque est importante : elle fait ressortir, d'une part, cette loi du silence qui dissocie le pouvoir-dire du savoir-dire, l'usage de la possession de la parole, introduisant par là un clivage qui la déflore, qui lui fait perdre son innocence et sa neutralité ; d'autre part, l'aspect « sanction > de l'initiation qui vient informer rituellement ce qui était déjà socialement inscrit, en démarquant et remarquant selon une problématique de l'expression : dire et ne pas dire.

Le caractère différé et différent du \& sevrage sociologique * selon les sociétés considérées, sans pour autant infirmer tout à fait la thèse de S. Moscovici - on peut finalement admettre que des sociétés puissent rejouer sur le plan du symbolique des situations sociales déjà inscrites -, en limite pourtant la portée explicative. Car si des rituels attestent sans aucune ambiguïté $\mathrm{l}$ " arrachement $\gg$, le \& transfert », le « partage sexuel », on ne peut réduire l'initiation à ces seuls moments et à ce seul sens : le passage à l'état adulte, le devenir homme ne sont pas en tout cas sociologiquement neutres, même si les discours et la théorie locale en nient la portée et la dimension sociales. Le culturalisme, voire le psychologisme, qui sous-tend l'interprétation de S. Moscovici, et qui prend le rituel initiatique au mot, négligeant par là ses antécédents et prolongements, les mouvements de va-et-vient entre le bois sacré et le village, oblitère en quelque

50. J. RABAIn-ZEMPLENI, op. cit., 1975, p. 424-425. 
sorte le rapport social fondamental (du moins pour les sociétés lignagères) qui joue, d'une manière grossie et amplifiée, à l'intérieur du cadre initiatique : celui d'aînés/cadets, les uns acquérant, par le biais des rites qu'ils contrôlent et légitiment, la maîtrise de la force de travail des autres.

Sans pousser l'hypothèse jusqu'à ce point, R. Girard (1972) insiste pourtant sur la problématique de l'écart et des différences qui marque selon lui tout rite de passage, tout rite d'initiation. Dans la société primitive, le moindre changement peut, pour un individu, apporter une crise majeure dans la mesure où il est perte momentanée de différences et réduction sociologique de l'écart, donc gain de violence. Partant de la notion de crise sacrificielle qui est définie par R. Girard comme une crise des différences, "c'est-à-dire de l'ordre culturel dans son ensemble » (qui n'est rien d'autre qu' « un système organisé de différences 》, puisque ce sont "les écarts différentiels qui donnent aux individus leur identité, qui leur permettent de se situer les uns par rapport aux autres ${ }^{51}$ ), il s'agit, par le biais de l'initiation, de faire l'économie d'une crise sacrificielle en écartant, en isolant, en marginalisant les individus en instance de passage : ils sont assimilés aux victimes d'une épidémie ou à des criminels qui risquent de répandre la violence autour d'eux ${ }^{52}$, donc rejetés mais aussi maîtrisés afin de prévenir la contagion. - Si terrifiante qu'elle soit, la perspective de passage n'est pourtant pas sans espoir. C'est à travers la perte généralisée des différences et la violence universelle, c'est à travers la crise sacrificielle et par son intermédiaire que la communauté, jadis, a débouché sur l'ordre différencié. La crise est la même et on peut espérer qu'elle aboutira au même résultat, à une instauration ou à une restauration des différences, c'est-à-dire, dans le cas des néophytes, à l'acquisition du nouveau statut ambitionné par eux. Ce dénouement favorable dépend d'abord de la violence souveraine, mais la communauté pense qu'elle peut $y$ contribuer. Elle va essayer de canaliser l'énergie maléfique dans les chemins que la collectivité a frayés. Pour que le résultat final soit le même que la première fois, pour mettre les chances du côté de la communauté, il faut reproduire, d'instant en instant, tout ce qui s'est produit cette première fois, il faut faire parcourir aux néophytes toutes les étapes de la crise sacrificielle, telles qu'on se les remémore,

51. R. GIRARD, op. cit., 1972 , p. 76 et s.

52. Ibid., p. 390 . 
il faut couler l'expérience actuelle dans le moule de l'expérience de jadis [...] Tel est le projet fondamental des rites de passage ${ }^{\text {ss }}$. ,

La perte du nom, la relecture de l'histoire personnelle par les aînés, la confession, la transgression parfois autorisée, quelquefois imposée, les secrets et la consigne du silence, l'atmosphère de terreur sacrée, la mise en quarantaine, les tortures et les blessures rituelles, etc., visent à conjurer la violence qui naît de la perte de différences, par cet exercice d'une violence différenciée et différée, localisée et socialisée : « Les rites de passage visent donc à structurer sur le modèle de la crise originelle toute crise potentielle, occasionnée par une perte quelconque de différence. Il s'agit de transformer en certitude l'incertitude terrifiante qui accompagne toujours l'apparition de la violence contagieuse ${ }^{54}$.

Cette interprétation hyperfonctionnaliste a le mérite de mettre en évidence, bien qu'il ne soit pas explicitement formulé, le processus de déculturation ${ }^{\mathrm{ss}}$ et de reculturation qui accompagne et/ou qui caractérise l'initiation, et qui l'identifie en somme à une de ces « institutions totalitaires » dont parle E. Goffman et qu'il définit ainsi : «Le caractère essentiel des institutions totalitaires est qu'elles appliquent à l'homme un traitement collectif conforme à un système d'organisation bureaucratique qui prend en charge tous ses besoins, quelles que soient en l'occurrence la nécessité ou l'efficacité de ce système [...] Lorsque les individus sont manipulés en groupe, ils sont généralement placés sous la responsabilité d'un personnel dont la tâche principale n'est pas de diriger ou de contrôler périodiquement le travail - cas fréquent dans les relations employeurs-employés -, mais plutôt de surveiller, c'est-à-dire de veiller à ce que chacun accomplisse la tâche qui lui a été impartie dans des conditions telles que toute infraction commise par un individu paraisse perpétuellement offerte aux regards par le contraste qu'elle offre avec le comportement des autres ${ }^{\text {se }}$. $\$$ Telle est en gros la situation sociologique dans laquelle se trouvent placés les initiés : la réclusion momentanée dans les enclos initiatiques, la « mise au secret » donc, la prise en charge des besoins élémentaires et l'administration des

53. Ibid., p. 392.

54. Ibid, p. 394.

55. Dans certaines sociétés, le futur initié a’a plus ni nom, ni passé, nı liens de parenté, ni droits d'aucune sorte. Il est réduit à l'état de chose informe et innommable (ibid., p. 391).

56. E. Goffman, Asiles..., op. cit., 1968, p. $45-54$. 
sanctions par un seul et même groupe de « surveillance \(les aînés sociaux), les techniques de mortification (isolement, mise en condition, dépouillement, agressions physiques et morales), etc., accusent cette dimension totalitaire de l'initiation. Il s'agit de normaliser, pour reprendre la terminologie de R. Girard, les écarts et les différences, de faire en sorte qu'ils soient la loi pour tous, même si en fait ils ne sont la loi que pour quelques-uns - précisément ceux qui, par leur position généalogique, détiennent le pouvoir réel et tirent profit, tant sur le plan symbolique et rituel qu'économique, de leur manipulation et de leur uniformisation idéologiques.

$\mathrm{La}$ ruse de la « raison initiatique > consiste précisément, sous le sceau du secret et du silence, à dissimuler des rapports de forces et de pouvoir, voire des rapports de production, à les rendre donc ni assignables ni contestables. $\mathrm{La}$ mise au silence, le devoir-taire, la marginalisation des initiés peuvent certes traduire une représentation du changement de statut it d'état comme étant impur ou menaçant pour l'ordre social - il s'agit de se prémunir de la contagion liée à la « perte des différences $>$, mais ils peuvent aussi bien et d'une façon plus positive accuser une tentative, une technique de soumission, de coercition des groupes cadets qui se trouvent, selon les cas et pendant une certaine période, rituellement et économiquement assujettis à leurs aînés sociaux.

Le poro des Sénoufo, et notamment le dernier cycle, le tyologo, le montre assez clairement : après un rituel assez complexe et jusqu'alors peu connu, les tyolobélé (ou porobélé, les initiés) sont soumis pendant sept ans à l'autorité du chef de bois sacré (sinzangfolo) qui, en pays kiembara, est souvent le même que le chef de village (kabafolo, un des descendants utérins du fondateur du village). Outre les charges rituelles et cultuelles qui leur incombent (principalement les services funéraires et les manipulations de masques), les porobélé doivent, selon les régions et surtout selon les groupes ethniques (trois jours chez les Kiembara), travailler un certain nombre de jours par semaine sur le champ collectif du village (segbo, appartenant au lignage fondateur), ou sur les champs des dignitaires du poro (chefs de quartier et de matrilignage), et * payer $\gg$ des amendes rituelles aux vieux du village. La fréquence et le taux de celles-ci souvent évaluées en poules ou poulets, depuis quelque temps et pour certains villages de la périphérie de Korhogo en numéraire - sont pour une part fonction des besoins cultuels des anciens (sacrifices aux ancêtres, funérailles et renouveau des funérailles $\gg$ ) et/ou des 
« fautes rituelles》de certains initiés, collectivement assumées; pour une autre part, des revenus propres que chaque initié tire de la parcelle (tiangonion) qui lui est attribuée quelque temps avant ou au moment de son entrée dans le bois sacré (sinzang). S'il y a donc, au bout du compte, une redistribution égalitaire, parce que proportionnelle, d'une part des revenus de chacun, le statut économique des initiés reste quant à lui profondément inégalitaire - ne serait-ce que par le mécanisme d'attribution des parcelles (tiangonion), dont Ia superficie et la valeur culturale varient suivant le capital foncier des quartiers ou des lignages - et le versement des amendes concourt à le reproduire et à l'ancrer socialement. Dans le bois sacré, mais seulement là, là où se paient les amendes, c'est-à-dire dans un lieu protégé et fermé aux regards du village et du quotidien, pour des fins rituelles et religieuses, la « richesse 》 se dévoile : elle se donne à voir mais ne se dit pas. La loi sociale s'affirme et se reproduit ici, dans ce périmètre de silences et de secrets, par cet impôt symbolique qui situe chacun aux yeux de tous et qui est signe de position dans l'ordre lignager.

Les prestations en travail fournies par les tyolobélé traduisent aussi, mais cette fois du côté des ânés, des inégalités sociales et économiques, contrairement aux discours locaux qui insistent sur leur finalité communautaire et coopérative. En effet, l'utilisation de cette force de travail, bien que théoriquement et pour tous les aînés disponible (au moins trois jours par semaine), doit être rétribuée en nourriture : \& Les vieux invitent le poro à venir travailler sur leurs champs. \& L Roussel fait remarquer que cette contre-prestation alimentaire est souvent onéreuse et que l' « invitation \$ d'un " poro de trente jeunes gens représente une dépense en nature ou en espèce d'environ 5000 C.F.A. ${ }^{\text {s7 }}$ >. \& C'est-à-dire, ajoute-t-il plus loin, que tous les ayants droit ne se trouvent pas toujours en mesure de faire appel à la main-d'œuvre d'un poro. Ils peuvent dans ce cas abandonner leur tour au profir d'un autre vieux; mais il semble que cette cession aille très précisément à l'opposé du sens de l'institution. Au lieu de fournir à tous les notables, chefs de quartier ou de famille, une force de travail égale, cette pratique conduisait à créer ou à aggraver les inégalités économiques. \ Nos propres observations sur ce point ont permis d'évaluer celles-ci et de constater le poids tant social qu'économique que représente l'institution du champ collectif

57. L. ROuSSEL, op. cit., 1965 , p. 54. 
(segbo), dont le propriétaire est le lignage fondateur du village qui monopolise pratiquement l'ensemble de la force de travail du poro, empêchant par là, et par la surenchère des contre-prestations alimentaires, le « décollage » économique de certains quartiers ou de certains lignages - ce qui accuse donc leur dépendance à l'égard du lignage fondateur et de ce fait dominant.

Ces quelques remarques tendent à montrer, du moins pour les Sénoufo, que l'appareil iniriatique permet, pour un certain temps, de disposer d'une force de travail importante, permanente, situable et « docile \$, même si elle n'est pas distribuée d'une façon égalitaire. La mise en quarantaine des initiés, justifiée idéologiquement par leur changement de statut, sert finalement les intérêts économiques et politiques des groupes dominants du village. Le régime des sanctions et des amendes, en court-circuitant a priori toute forme de déviance et de contestation, favorise l'endettement, la dépendance économique et reproduit une hiérarchie fondée sur la structure lignagère. Si la « sortie \ du bois sacré, elle-même rituellement marquée, traduit théoriquement l'accès à l'état d'adulte, au statut d'ancien, elle n'affirme pas pour autant une égalité entre les « sortants », devenus les aînés de la classe suivante mais soumis eux-mêmes à cette loi lignagère qui semble indiquer qu'un aîné peut être un cadet social. L'erreur des interprétations classiques de l'initiation réside peut-être dans le fait qu'elles l'aient étudiée séparément des processus sociaux, économiques et politiques qui la fondent et qu'elle contribue à reproduire. L. Krader insiste précisément sur ce point à propos du poro des Kpelle du Liberia et de Guinée, proche par sa structure et son contenu de celui des Sénoufo : \& L'initiation comportait la transmission des secrets d'ordre religieux de la société et l'apprentissage des vertus mâles kpelle : obéissance et force d'âme [...] Les membres en vue du poro pouvaient être admis dans des organisations plus secrètes encore, maitrisant des mystères plus profonds et investis d'une plus grande autorité sacrée. Au sein du système des sociétés secrètes, les magiciens étaient initiés à leur art propre, les simples membres aux arts nécessaires à l'activité quotidienne, les fils de nobles et les chefs aux arts utiles à leur condition, cérémonial de cour, règles de succession, et avaient accès aux traditions tribales réservées à leur rang et aux arcanes de la politique. Ainsi le poro et les sociétés secrètes apparentées constituaient des éléments opérationnels dans l'organisation sociale et politique des Kpelle ${ }^{38}$. > D. Paulme

58. L. KRADER, Formation of the State, op. cit., 1968, p. 39. 
fait des remarques semblables : « L'étude des rapports entre initiation tribale et classes d'âge, telle qu'elle se présente notamment chez les Bobo, pourrait être prolongée par une comparaison avec l'initiation de type poro, en usage dans plusieurs sociétés du Liberia ou de la Sierra Leone. Dans les deux cas, tous les hommes sans exception (toutes les filles dans le bundo symétrique du poro) sont soumis à la première épreuve, qui a lieu en forêt, à l'écart des femmes et des. enfants, et se termine par une rentrée solennelle au village, véritable naissance à l'âge d'homme. Dans les deux cas également, l'essentiel de l'enseignement alors donné porte sur les masques, leur nature humaine, l'origine et le rôle de chacun, la langue secrète; c'est par le canal des masques que sont transmises les lois de la tribu. Là cessent les ressemblances : dans le poro des Mende et de leurs voisins, un enseignement ultérieur n'est pas distribué à tous, seuls les membres de certains lignages peuvent espérer atteindre un grade supérieur, se voir investis d'une dignité qu'ils recueilleront par héritage, à la mort d'un aîné de leurs parents. Le conseil du poro, dont les délibérations sont secrètes et qui détient en définitive le pouvoir, est donc aux mains de quelques individus - de quelques lignages - dont le chef de village ou de région ne fait qu'exécuter les décisions. Le cadre démocratique de la communauté villageoise, tel qu'il se présente chez les Bobo et nombre de sociétés de la savane soudanaise, est ici rejeté au profit d'une minorité ${ }^{\text {so. }}$.

Dans ces exemples, le jeu du secret paraît organiser, ou plutôt souligner, une hiérarchie sociale fondée sur la structure lignagère. A chaque groupe, à chaque lignage ses secrets et ses silences qui marquent autant de frontières et de distances. Ce qui semble finalement inscrire le statut est moins la communication du ou des savoirs qui lui sont associés que leur rétention. Une telle stratégie n'est pas particulière aux sociétés traditionnelles, et l'on pourrait dans notre propre société chercher et trouver des groupes ou des catégories socio-professionnelles qui agissent de même, c'est-à-dire qui utilisent le couple savoir/secret comme moyen de consolider un statut, de renforcer un pouvoir et de maintenir une hiérarchie. C'est ce qu'a très bien senti $\mathrm{R}$. Merle dans L'Ile lorsqu'il écrit : \& Le secret n'était pas utile. Il n'avait qu'une valeur hiérarchique : le chef exerçait un privilège de chef en laissant ses subordonnés ignorer ce que le chef savait. Ainsi le secret de la destination du Blossom avait mis une

59. D. Paulme, op. cit., 1971, p. 22. 
distance entre Purcell et lui, et ce secret, partagé maintenant entre le capitaine et le second, maintenait la même distance entre les officiers et l'équipage ${ }^{60}$. Par ailleurs, nous avons vu que, pour les tendeurs du plateau ardennais, il s'agissait de se réserver socialement un savoir - qui pouvait être connu de tous et partagé par tous - en monopolisant le droit à la nomination et en instituant un régime de secrets qui en validait paradoxalement la portée sociale. D'une façon quasi exemplaire, la relation psychanalytique fait ressortir ce mécanisme :

60. R. MERLE, L'lle, Gallimard, Paris, 1962, p. 71.

Dans ce domaine (celui de la littérature qui, comme source d'information, comme valeur démonstrative ou comme repère d'une problématique, n'est pas à négliger), les auteurs anglo-saxons de la seconde moitié du $\mathrm{XIX}^{\circ}$ siècle ont, peut-être plus que d'autres, souligné et volontiers décrit ce qui est de l'ordre de l'indicible, du secret, du silence, du masque et du camouflage. Je pense surtout à Nathaniel Hawthorne qui, dans ses contes (Le Voile noir, La Marque sur le visage, Le Manteau de Lady Eléonore) ou dans ses romans (Valjoie, La Leture écarlate), fait du secret et de son dévoilement le centre de l'intrigue et le moteur de l'action des personnages. Très proche, Herman Melville érige en principe la thématique du voile, qui fournit la trame épaisse de ses ceuvres, insistant sur la manière dont les choses peuvent être dissimulées au regard d'éventuels observateurs (différemment de Hawthorne où la révélation est capitale, et à l'inverse d'Edgar 'Poe dans La Lettre volée) : c Toutes choses visibles, fait-il dire à Achab dans Moby Dick, ne sont que des masques de carton-pâte $\gg$, masques qu'il s'agit de déchirer. Mais la blancheur de Moby Dick, sa transparence, de-ci de-là cernée et affirmée d'une façon docte, à la limite pédante (cf. l'ouverture du roman et les chapitres d'érudition sur la cétologie), s'avère bien grise et opaque au bout de la course. Le sens de la quête d'Achab s'estompe au fur et à mesure : * Que je le veuille ou non, se dit Starbuck, le second du Pequod, l'indicible m'a lié à Achab et me remorque avec un câble pour lequel il n'est point de couteau. $\$$ Là, il n'y a plus de secrets d découvrir : Et peut-être qu'après tout il n'y a pas de secret $\gg$, écrit Melville à Hawthorne en 1851. Le secret et l'évident - le blanc-noir - sont à armes égales, s'interpénètrent, se dérobent et se dévorent. C'est l'ambiguïté des apparences qui compte, la grisaille du quotidien (Bartleby l'écrivain), le silence (Benito Cereno, Billy Budd), le calme plat (Mardi), la façon dont les objets se voilent plutôt que ce qui est caché (Moby Dick, Mardi, Typee). La parole n'est que piège. Elle se défile au moment même où elle pourrait révéler, mais révéler quoi ? ( $\mathrm{La}$ parole manque à Billy Budd lorsque le capitaine Vere l'invite à dire son innocence, son silence le condamne à la pendaison, détruit l'accusateur Claggart, mais sauve les rapports de pouvoir; le silence de Benito Cereno, qui calque celui, légitime, du nègre Babo, son esclave, interroge le capitaine Delano sur le commandement du San Dominick, sur la nature du pouvoir, sur la relation maître-esclave). Le savoir est cannibale (Typee) : il mange du pouvoir, la chose au monde la moins partagée et la moins sécable qu'il faut savoir économiser par le silence (cf. le savoir égoïste \ d'Achab sur Moby Dick; celui du Danois dans Billy Budd; celui d'Elie, le prophète, à propos. de la jambe d'Achab, absente, mais peut-être raison de sa quête sauvage - comme celle de Tommo dans Typee, par contre trop présente par son enflure, peut-être raison de sa captivité chez les Tai-pi cannibales : Tout! Hein? Etes-vous sûr de tout savoir? Vraiment tout? \$). 
la position et le pouvoir de l'analyste se définissent et résident justement dans la rétention de sa parole et la suspension de son savoir ${ }^{61}$.

Dans un ouvrage récent, $P$. Roqueplo, partant d'une étude rapide des notions de formation, d'information et de vulgarisation, pose le problème du savoir et de la compétence en termes politiques : dans notre société, il existe à tous les niveaux, et face à l'inflation de l'information et de la vulgarisation, un réflexe de rétention du savoir, "c'est-à-dire, écrit-il, de protection de son propre savoir et de sa propre place dans la biérarchie sociale. Dans ces conditions, il est finalement assez dérisoire de ne considérer le partage du savoir qu'en termes pédagogiques et " culturels ". Ce dont il s'agit en partageant ou en ne partageant pas le savoir, c'est en fait de partager ou de ne pas partager un certain pouvoir. Le problème n'est pas d'abord un problème de communication mais un problème de conflit et de transgression. Un problème de structure sociale. Un problème politique $^{62} \gg$. Cette parenthèse faite, il est bien certain, pour en revenir à l'initiation et notamment au poro, que la vulgarisation de ses secrets, la mise en évidence de son organisation interne et des épreuves qui marquent le passage entre les degrés risqueraient sans doute, par la démocratisation des formes et du contenu de l' a en. seignement initiatique $\gg$, d'ébranler la structure traditionnelle de l'autorité en enlevant à cette dernière le pouvoir de contrôler, d'imposer et de diffuser les champs et la sélection des significations. Toutefois, dès lors que l'on envisage cette question de la transmission des savoirs tribaux, il n'est pas évident que l'expérience initiatique en soit le canal privilégié, assure donc une formation, permette finalement d'acquérir connaissance et compétence.

Pour D. Zahan et pour une certaine école ethnologique française, la fonction de l'initiation est pédagogique : il s'agit de révéler les significations cachées, celles des masques, des parures, des rites, des corps, de l'univers. "Une première constatation apparaît avec une netteté remarquable dès qu'on ouvre le dossier de l'initiation, c'est que celle-ci constitue, avant tout, un enseignement progressif destiné à familiariser la personne humaine avec les significations de son propre corps et avec le sens qu'elle doit donner à l'univers

61. Cf. R. CASTEL, Le Psychanalysme, op. cit., 1973. Dans un domaine proche, il y aurait beaucoup à dire sur le secret médical et sur la consigne du silence en milieu hospitalier.

62. P. ROQUEPLO, op. cit., 1974, p. 223. 
ambiant ${ }^{63}$. Pour P. Clastres, \& il s'agit de ne pas perdre la mémoire du secret confié par la tribu, la mémoire de ce savoir dont sont désormais dépositaires les jeunes initiés ${ }^{84} \gg$. On pourrait à loisir citer d'autres textes, glanés de-ci de-là dans les monographies, qui insistent sur le côté « école de brousse » de l'initiation. En règle générale, les auteurs s'accordent à dire qu'il s'agit d'un processus pédagogique essentiel visant à expliciter le dispositif symbolique utilisé par la société. L'avancement dans la connaissance et la possession des secrets traduiraient donc une hiérarchie interne qui peut être organisée de deux manières : ou bien cette connaissance est distributive et sélective (comme dans le poro) et se transmet selon un principe d'appartenance lignagère, chaque groupe se définissant dans un même temps par rapport aux autres, par le « paquet \$ de secrets qu'il détient; ou bien elle est progressive et à tendance égalitaire et se transmet selon le principe des générations, chacun pouvant a priori accéder selon son âge biologique au savoir « suprême ». Ces deux processus de transmission du savoir peuvent bien entendu coexister dans une même société (chaque groupe distinct étant luimême soumis à la hiérarchie des âges), mais n'est-il pas plus intéressant de noter que, dans bien des cas, ce savoir parait être un leurre puisque chaque groupe d'âge ou chaque groupe en corps affirme son statut, sa position et son pouvoir moins par la mise en pratique d'un savoir qu'il détient et contrôle que par sa suspension et sa rétention, par les secrets qui l'entourent et qui agissent comme barrière et niveau ? Car l'essentiel n'est-il pas plutôt de cacher? Ce qui en somme expliquerait ce « terrorisme du silence \$ souvent observé dans les rites initiatiques. C. Lévi-Strauss fait justement remarquer, à propos des Bororo, que le secret donne plus de réalité à ce qui est caché ${ }^{65}$. S. Moscovici souligne l'aspect trompeur de cette connaissance secrète : " Moins appréhension de l'inconnu que saisie de l'interdit, moins découverte du réel que déchiffrement de ses déguisements, elle est lumière et libération pour celui qui a acquis le droit de l'approcher, à condition de demeurer terreur et obscurité pour celui à qui ce droit est refusé. Ceci traduit la duplicité de la connaissance, inséparable de la dissimulation [...] Tout ce qui est considéré comme vrai doit être masqué par des histoires, des légen-

63. D. ZAHAN, op. cit., 1970 , p. 91.

64. P. Clastras, op. cit., 1974 , p. 157.

65. Tristes Tropiques, U.G.E., 10/18, Paris, p. 209-212. 
des, des actions ad mulierem, afin d'empêcher que sa transparence ne nuise à la vie publique. Mais dans ce domaine on n'atteint jamais la clarté, et le couple de ceux qui savent et de ceux qui ne savent pas est lié par une incertitude commune : ceux (ou plutôt celles) qui sont censés ignorer ne connaissent-ils pas la vérité ? Ceux qui connaissent la vérité n'en ignorent-ils pas l'autre face? La réalité sociale se profile comme envers d'une cérémonie. Le secret demandé tranche le dilemme sans le résoudre, rend la convention efficace sans pourtant lui assurer un fondement substantiel, puisqu'il est ruse, faux-fuyant, equivoque ${ }^{\text {se }}$. L'endoctrinement initiatique est d'abord et surtout apprentissage du camouflage, où ce qui importe est plus la dissimulation que son objet.

L. Perrois, dans sa monographie sur la circoncision bakota, insiste aussi sur le « vide » du savoir initiatique : "Tout d'abord, il faut se demander [au sujet de l'initiation bakota] s'il s'agit de véritables " initiations " avec l'enseignement élaboré d'un corpus de connaissances ésotériques. $A$ vrai dire, non; ces connaissances-là, car il y en a certainement (médicaments, connaissance exacte du symbolisme), s'acquièrent tout au long de la vie, un peu au hasard des amitiés avec un vieux ou une vieille qui vous prend comme disciple et héritier. Elles ne s'obtiennent pas comme le savoir scientifique occidental : c'est toute une imprégnation lente et mesurée qui envahit peu à peu celui qui deviendra un nganga, un homme de l'art, riche de l'expérience de toute une vie. Donc point d'école de brousse. L'initiation est plutôt un passage de l'état d'enfant ignorant et incapable à celui d'homme qui pourra accéder à la connaissance. C'est donc plus une admission qu'une initiation : les rites donnent accès à l'association, ils transforment le candidat de façon à ce qu'il soit capable désormais de voir toutes les pratiques, d'y participer et surtout d'en bénéficier ${ }^{67}$. $\gg \mathrm{La}$ prudence initiale de ce texte traduit assez bien la gêne des observateurs contemporains devant une institution qui reste encore marquée par cette aura mystique dans. laquelle la tradition ethnologique la maintenait ${ }^{\text {es }}$. Bien que lappareil symbolique soit ici en quelque sorte condensé et souvent clairement délimité, il n'est pas certain qu'il puisse tout dire surr la société qui l'utilise, même si les discours locaux le prétendent.

66. S. Moscovici, op. cit., 1972, p. 303.

67. L. Perrols, La Circoncision bakota (Gabon), op. cit., 1968, p. 70.

68. Cf. les travaux de M. Leiris, M. Griaule, B. Holas, D. Zahan, etc. 
La fascination qu'il exerce toujours sur les ethnographes se mesure en fait à la façon dont il se dérobe à leurs regards. Sorte de San Dominick sociologique, il guette et piège ses Delano dans l'entrelacs de son gréement ${ }^{69}$. Ce qui est caché, le secret, attire, et bon nombre de sociétés ont compris le parti tant économique que politique qu'elles pouvaient tirer de cette curiosité obsessionnelle, même si, au bout du compte, elles n'offraient pas l'équivalent supputé. Ce qui est vrai pour l'ethnographe peut également l'être pour le néophyte autochtone pour qui la soumission, la docilité, la conformité garantissent l'accès à la \& connaissance profonde > et accordent l'identité, souvent chèrement payée, comme chez les Sénoufo, et a fortiori durement défendue. La futilité du contenu des secrets a quelquefois de quoi surprendre et de quoi ridiculiser les efforts entrepris pour y accéder. Je me souviens notamment de cette remarque que fit mon interprète à sa sortie du bois sacré : « Vraiment, il faut que les jeunes Sénoufo soient des cons pour croire que cette mascarade leur apprend quelque chose ! On savait tout avant!, Certes, mais ce savoir n'était pas socialement reconnu et validé. Contesté individuellement, quelquefois collectivement (notamment lors des cultes messianiques de Massa et de Moussa), le poro et ses secrets continuent néanmoins de distribuer des cartes d'identité sans lesquelles on ne peut ni se mouvoir, ni écouter, ni parler au sein de l'univers villageois. L. Perrois banalise, à mon sens avec raison, l'expérience pédagogique de l'initiation, en insistant d'une part sur l'importance des circuits et des instances pédagogiques parallèles, et en soulignant d'autre part son aspect de mise en scène sociale. En définitive, il s'agirait moins de transmettre un savoir secret, de donner la signification des rites et des symboles - conception cryptologique justement critiquée par D. Sperber ${ }^{70}$ - que de placer chacun face à un appareil symbolique qu'il peut manipuler mais dont il ignore le sens, celui-ci étant, mais n'est-ce pas le but recherché, sans cesse différé et dissimulé.

De fait, le poro des Sénoufo, souvent cité comme un modèle de l'enseignement ésotérique africain, s'avère sur ce plan, dès qu'on l'approche, curieusement décevant. Le scénario est en tout cas classique : on « tue symboliquement les néophytes par une série

69. Cf. l'admirable nouvelle de H. Melvilis, Benito Cereno, Gallimard, Paris, 1951.

70. Le Symbolisme en général, op. cit., 1974, p. 29-35. 
d'épreuves de mortification (fustigation, aspersion d'eau épicée mélangée à des graviers sur le corps nu des initiés, ingurgitation de liquide vomitif, etc.), avant de les faire renaître dans le vagin de kabatiéléo ${ }^{71}$, représenté sans aucune ambiguïté par un boyau boueux qui débouche dans le ventre de la « vieille » (tiékpag), enclos de rondins sur le périmètre duquel se dressent deux ou trois cases rondes servant d'abri aux initiés. L'enseignement proprement dit consiste, au petit matin, une fois que les néophytes ont reçu leur nom secret ${ }^{\text {T }}$ et que les éléments de l'appareil liturgique ont été montrés et nommés, à présenter un stock d'objets usuels, quotidiens, notamment prélevés dans l'environnement culinaire (dont la manipulation est, au village, réservée aux femmes), que l'on redéfinit et renomme suivant une symbolique sexuelle. Ces noms communiqués dans le bois sacré sont mis en forme dans des chants appris par la suite et produits en public lors des funérailles. Il n'y a donc pas de langue secrète, comme l'affirmait avec une certaine outrecuidance $\mathrm{B}$. Holas ${ }^{73}$, mais constitution d'un ensemble limité de termes dont les référents appartiennent au domaine culinaire, celui des femmes, et subissent sous l'impulsion des hommes un glissement sémantique. Le riz, les condiments, le pilon, le mortier, etc., sont donc, dans le bois sacré, nommés différemment sans qu'on puisse dire qu'il existe des règles métaphoriques précises; car si le stock d'objets reste à peu près constant, il existe de grandes variations lexicales selon les bois sacrés.

Par la suite, lors de \& séances spéciales \$, la manipulation de certains masques, les chants et danses du poro, l'utilisation des instruments (tambours, mirlitons, trompes, etc.) sont enseignés aux nouveaux initiés dans le cadre du bois sacré. Telle est en gros la * vocation pédagogique de l'initiation sénoufo : ni explication, ni interprétation, ni commentaire, mais révélation brutale d'instruments liturgiques, apprentissage progressif de leur syntaxe, mais

71. Textuellement vieille mère du village $\gg$; principe socio-gonique et socio-régulateur, manifestation d'une même essence divine dont l'autre face est représentée par koulotioléo ou koulo syolo, « vieille femme de l'univers \$, principe transcendant et cosmique.

72. Ce nom est attribué par les dignizaires du poro, à la suite de longues délibérations où sont envisagés les antécédents, l'histoire, les qualités et défauts du néophyte, résumés dans une formule métaphorique. Pour un même village, ces noms, à l'inverse des anthroponymes, ne constituent pas un ensemble défini et limité.

73. Les Sénoufo..., op. cit., 1966, p. 151-15s. 
occultation de leur sens. Un sens réservé selon les uns aux anciens, aux dignitaires du poro, perdu selon les autres par quelque ancêtre; un sens, en tout cas, peu déterminant et opératoire, car son ignorance n'empêche nullement de manipuler l'appareil symbolique, d'acquérir le statut d'initié et de s'inscrire dans la hiérarchie de l'institution. Il n'y a pas, à ma connaissance, cette longue méditation sur la vie et sur la mort, cette profonde réflexion sur les mythes et les rituels dont parle $\mathrm{D}$. Zahan. La quête et la découverte du sens se jouent ailleurs et à d'autres moments, tout au long de la vie, un peu au hasard, comme le soulignait $\mathrm{L}$. Perrois dans le texte précédemment cité, et comme l'affirme d'une façon plus théorique $D$. Sperber : " La vie symbolique de l'individu ne se divise pas nettement entre une période d'apprentissage et une période d'utilisation d'un dispositif établi. Le symbolisme, ajoute-t-il, parce qu'il est cognitif, reste toute la vie un dispositif d'apprentissage ${ }^{74}$. $\gg$ L'entrée dans le bois sacré n'est pas, dans notre cas, accè's à la connaissance, mais traduit plutôt un passage sociologique. Le périmètre de l'enclos initiatique est plus social et politique que culturel. Même si les symboles qui y sont présentés ou manipulés se réfèrent à une mythologie ou une théorie, celle-ci n'est ni explicite ni explicitée. Elle reste soigneusement dissimulée. Car il importe toujours de cacher pour mieux se démarquer.

\section{De la parole retenue à la parole tenue}

L'initiation, telle qu'elle vient d'être envisagée, serait donc plus un dispositif de reconnaissance que de connaissance, de pouvoir que de savoir. La révélation même futile des secrets, leur partage par une catégorie de la population suffisent à fracturer l'univers social de la communication, d'une part géographiquement (opposition village/ bois sacré), d'autre part sociologiquement (initiés/non-initiés), et à informer des structures de subordination (aînés/cadets). Le passage des épreuves, la mise au secret et la réclusion (enclos initiatique), en imposant un devoir-taire, accordent paradoxalement un pouvoir-

74. D. SPERBER, op. cit., 1974, p. 101. 
dire, mettent en tout cas les jeunes gens en situation sociale de dire et d'écouter, en somme de participer.

Cette dialectique du silence et de la parole, sa dimension à la limite stratégique et politique, prend tout son sens et son poids lors de situations conflictuelles où la prestation de serments (rite d'allégeance à l'organisation ou à la secte) condamne l'individu, par la parole donnée, au silence. En le liant à un groupe donné et en éclaircissant les frontières socio-culturelles entre les divers groupes, le serment joue un rôle déterminant dans la création et le maintien d'une solidarité sociale ainsi que dans la prévention de l'anomie. Les modes de sanction qui interviennent lors de la rupture du serment, souvent exemplaires, accusent cette allégeance quasi totale de l'individu au groupe et confèrent un haut degré de conformité sociale. Cela est important lors de situations politiques conflictuelles, où des individus sans envergure et conscience politiques peuvent être amenés à défier les lois, à recourir à la violence physique, à être emprisonnés et torturés. On peut notamment en voir les effets dans le fameux serment Mau-Mau. Utilisé dès 1920 par les Kikuyu squat-. terisés dans un contexte de lutte anticoloniale, le serment dit de l'unité avait pour objectif de rallier l'ensemble des Kikuyu pour les engager au besoin dans des actions politiques, voire militaires, visant la restitution des terres aliénées par les colons britanniques au début du siècle. La culture traditionnelle fournissait à cet égard des modèles de prestation de serments et nous verrons que les différents leaders leur empruntèrent certains éléments. Avec l'arrivée des. Européens, les serments, qui dans la société traditionnelle avaient une fonction d'intégration, furent vidés de leur contenu et sociologiquement déconnectés. Les missionnaires les condamnèrent purement et simplement, voyant dans leur déroulement cérémoniel une preuve de l'atavisme et de la sauvagerie des \& terrifiants \ Kikuyu. L'attitude de l'administration coloniale fut plus nuancée et, de ce fait, plus ambiguë : bien que les condamnant, d'ailleurs plus par ignorance de leurs fonctions que par idéologie, elle exigeait que les notables kikuyu prêtent le serment d'allégeance à la couronne d'Angleterre lors des réunions des Local Native Councils. De ce fait, une certaine confusion apparut et les premières prestations du serment de loyauté et d'unité à la Kikuyu Central Asssociation (K.C.A.) ${ }^{75}$ en portèrent la marque. Ce serment était apparu

75. Organisation politique fondée en 1924 par Jesse Kariuki. Son influence était sensible dans le Nyéri et le Kort-Hall, c'est-à-dire dans des districts où 
dès 1924 : * Tous ceux qui connaissent mon peuple, remarque J.M. Kariuki, comprendront qu'il ait spontanément voulu sceller son unité par un serment. J'ai appris que c'est dans les districts kikuyu, et d'abord dans le Kiambu, qu'on commença à prêter serment. Et ce sans impulsion ni contrôle d'en haut. Le serment. n'était ni sophistiqué ni élaboré, et n'avait d'abord rien de choquant il ne fut repris que lentement et même difficilement; c'était un serment d'unité et de fraternité dans la lutte pour notre terre et notre indépendance. Il finit par se propager dans tout le pays, au sein d'un peuple n'ayant rien à espérer d'un Etat dominé par les Européens ${ }^{78}$.

Le serment de la K.C.A. comportair au départ peu d'éléments traditionnels et était en partie calqué sur celui qui se prêtait dans les Local Native Councils : ainsi le postulant prêtait serment sur la Bible - mais tenait en même temps une motte de terre humide qu'il pressait sur son nombril. Dès 1930, les emprunts à la culture traditionnelle se firent plus nets. On possède toutefois peu de renseignements sur cette période. C. G. Rosberg et J. Nottingham ${ }^{77}$ croient pouvoir établir que la Bible fut conservée jusqu'à la fin de la Seconde Guerre mondiale, où elle fut définitivement remplacée par une chèvre!

Voici un exemple du serment de l'unité de la K.C.A. Il fut prêté en 1952, soit quelques mois avant la révolte des Mau-Mau. Le développement de l'agitation dans les réserves kikuyu et l'intensité des conflits avec l'administration coloniale expliquent d'une certaine façon le nombre, le caractère normatif et idéologique des \& voux * qui y sont exprimés :

- La lampe tempête éclairait le visage des gardes armés de pangas $^{78}$ et de simis (épées). Devant nous, à droite, il y avait une arche faite de tiges de bananier, de tiges de maïs, de canne à sucre, maintenues par des lianes. On nous ordonna de retirer notre tunique,

la dégradation des conditions de vie était la plus sensible. Son programme comportait essentiellement des revendications foncières (restitution des terres aliénées), et les leaders de la K.C.A. envisageaient le recours à la violence comme une solution politique parfois nécessaire.

76. J. M. KARIUKI, Maw-Mau Detainee. The Account by a Kenya African of bis Experiences in Detention Camps, Oxford University Press, Nairobi, 1963, p. 22.

77. The Mytb of Mau-Mau, F. A. Praeger, New York, 1966.

78. Machettes d'origine européenne qui ont remplacé les traditionnelles bêches (kabiyu). 
notre montre, nos chaussures et tout autre objet d'origine européenne qui était en notre possession. L'officiant, son gros visage enduit de craie, entoura le poignet droit de chacune des sept personnes qui devaient être initiées d'une lanière découpée dans la peau crue d'une chèvre. On nous attacha les uns aux autres par les épaules et par les pieds avec les boyaux de la chèvre sacrifiée. Quelqu'un nous aspergea de bière indigène avec la bouche pendant qu'il nous baptisait en nous jetant des épis de millet mélangés à d'autres céréales. L'officiant nous piqua le majeur de la main droite avec une épine, jusqu'à ce que le sang apparût. Puis il nous apporta la poitrine d'un bouc avec le cœur encore attaché aux poumons et il la barbouilla avec notre sang. Il prit ensuite une gourde indigène pleine de sang, avec lequel il fit une croix sur notre front et sur toutes les principales jointures de notre corps en disant : "Que ce sang soit le symbole des membres fidèles et courageux de l'Unité Gikuyu et Mumbi $^{79}$; que ce sang vous rappelle que si vous dévoilez notre secret ou violez le serment, nous viendrons pour vous tailler en pièces à ces endroits marqués par ce sang! " On nous demanda ensuite de nous lécher mutuellement le sang de notre doigt et de répéter après l'officiant : "Si je révèle le secret de Gikuyu et Mumbi à une personne étrangère à l'Unité, que ce sang me tue! $\mathrm{Si}_{i}$ je révèle un seul des vœux du serment, que ce sang me tue! Si je mens, que ce sang me tue! "On nous ordonna de nous prendre la main droite et de passer en file sept fois sous l'arche. $A$ chaque passage, l'officiant coupait un morceau de boyau de chèvre, le réduisant en pièces, pendant que toute l'assemblée nous jetait l'anathème suivant : " Maudits! ainsi soyez coupés! Que le serment tue celui qui ment!" Alors, entourés par les morceaux d'intestin, nous nous tournâmes vers le mont Kenya et on déposa dans nos mains deux mottes de terre humide. On nous demanda de presser celle que tenait la main gauche sur le nombril. Ainsi nous prêtâmes serment :

“ Je (K. Njama) jure devant Dieu et devant tous ceux ici présents que...

1. Je ne révélerai jamais le secret du serment de la Kikuyu Central Association (K.C.A.) - celui de Gikuyu et Mumbi - celle [la K.C.A.] qui revendique la liberté et l'indépendance. S'il arrive que je le fasse, que ce serment me tue ! (Cette dernière

79. Du nom des ancêtres mythiques de la tribu. 
phrase devait être répétée après chaque vœu en même temps qu'il fallait frapper la poitrine du bouc.)

2. J'irai toujours au secours des membres de la société qui se trouvent en difficulté.

3. Si, à toute heure du jour ou de la nuit, je suis appelé à faire un travail pour la société, je le ferai.

4. Jamais je ne désobéirai aux chefs de la société.

5. Si l'on me demande de cacher des armes et des munitions, je le ferai.

6. Je donnerai de l'argent ou des vivres si la société m'ordonne de le faire.

7. Jamais je ne vendrai des terres aux Européens ou aux Asiatiques.

8. Jamais je ne coucherai avec une putain.

9. Jamais je ne mettrai une femme enceinte sans l'épouser.

10. Jamais je ne chercherai à divorcer ${ }^{80}$.

11. Jamais je ne permettrai le mariage entre Africains et Européens.

12. Jamais je ne tolérerai qu'aucune de mes filles ne soit excisée.

13. Jamais je ne boirai de la bière fabriquée par les Européens. Jamais je ne fumerai de leurs cigarettes.

14. Jamais je n'espionnerai ni ne trahirai mon peuple pour le gouvernement.

15. Jamais je n'aiderai les missionnaires et leur religion chrétienne qui détruit nos traditions.

16. Jamais je ne m'approprierai le bien d'un membre de la société.

17. J'obéirai à tout appel.

18. Jamais je ne laisserai une de nos revendications. Je les soutiendrai toutes jusqu'à ce que nos buts soient atteints.

19. Je paierai 62 sh. 50 cents et un bélier comme tribut à la société dès que je le pourrai.

20. Je suivrai toujours Jomo Kenyatta, notre chef."

Pendant que nous prêtions serment, nous piquâmes sept fois l'œil de la chèvre avec une épine, puis, à la fin, nous piquâmes sept pommes de Sodome. On nous fit ensuite, avec du sang mélangé à de la terre, une croix sur le front, ceci indiquant que nous étions dorénavant des membres à part entière de l'Unité Gikuyu et Mumbi, et l'on nous cria : "Toujours en avant, jamais en arrière!" On

80. Ce vœu peut surprendre, d'autant que le divorce était non seulement toléré, mais monnaie courante dans la société traditionnelle. 
reprit nos affaires et on remit nos vêtements. Chacun paya 2 sh. 50 cents pour l'enregistrement. L'officiant nous harangua : " Nous avons compris que les colons du Kenya sont le principal obstacle à nos buts. Ils ne veulent pas abandonner les profits qu'ils tirent des terres au prix de notre sang dans ce soi-disant " paradis blanc ". Certains d'entre vous ont dû payer une amende, voire même ont été emprisonnés pour avoir enfreint le règlement qui nous interdit de pénétrer sur les terres du colon, alors qu'ils allaient rendre visite à des parents qui y sont employés, ou bien y allaient chercher du travail. Nous ne pouvons plus tolérer cela plus longtemps. Nous n'aurons de cesse de le crier au gouvernement kényan, dont nous savons qu'il est à la solde des colons, jusqu'à ce que nous soyons écoutés, sinon jusqu'à ce que leurs tympans éclatent. Vous avez entendu dire que certains de nos membres ont été persécutés pour avoir prêté le serment Mau-Mau. C'est le même serment que vous avez aujourd'hui prêté. Vous êtes maintenant membres de ce Mau-Mau. Mais ne le dites jamais. Si vous le divulguez, le gouvernement vous emprisonnera et nous vous tuerons pour avoir violé le serment que vous avez prêté aujourd'hui. Tous nos membres sont dans la place, même dans les bureaux gouvernementaux." "

Cette cérémonie, rapportée par Karari $\mathrm{Njama}^{81}$ (qui fut lui-même général de la Land Freedom Army, armée Mau-Mau dirigée par Dedan Kimathi et réfugiée dès 1952 dans la forêt des Aberdare), fait ressortir l'atmosphère de terreur et la « violence sacrée 》 qui défrayèrent tant la chronique de l'époque et qui faisaient des participants des êtres à part, comme le fait remarquer $R$. Buijtenhuijs : « La solidarité nouvelle s'exprime d'abord de façon négative : les membres du mouvement faisaient véritablement " bande à part ", s'efforçant de marquer la distance entre eux-mêmes et les noninitiés. Un ostracisme total frappait, dans certaines communautés, villageois et urbains, ceux qui n'avaient pas prêté le serment ${ }^{82}$. $\gg$ On retrouve là une des fonctions du secret, d'ailleurs abondamment mentionnée dans les vœux : maintenir et accuser des distances sociales. Un autre point mérite d'être mentionné, c'est le caractère de véritable initiation donné à cette cérémonie, par la mobilisation de a l'arsenal

81. Cf. D. L. BarnetT et K. NJAMA, Mau-Mau from Within. Autobiography and Analysis of Kenya's Peasant Revolt, Monthly Review Press, New York, 1966, p. 117-119.

82. R. BuIJTEnhuIJ, Le Mouvement Mau-Mau..., op. cit., 1969, p. 285. 
spirituel et religieux des Kikuyu ». Buijtenhuijs n'hésite pas à parler de « cocktail sacré \$!

Le serment de l'unité, dans son déroulement cérémoniel, connut plusieurs variantes. Toutefois, les vœux exprimés restaient relativẹment homogènes. En apparence, le serment de l'unité révèle une tentative de recomposition, de reconstruction d'une forme d'organisation sociale sur la base d'éléments empruntés parmi les plus pertinents du système traditionnel. L'attitude requise pour signifier son appartenance à la Kikuyu Central Association, donc pour signifier que l'on avait prêté serment, est assez révélatrice : s'il arrivait en effet qu'un des nouveaux membres voulût savoir si la personne qu'il rencontrait appartenait elle aussi à l'Unité Gikuyu et Mumbi, il devait demander : « Où as-tu été circoncis? \$ Dans l'affirmative, la réponse adéquate, le mot de passe en quelque sorte, était : - J'ai été circoncis à Karimania par Karimania. \ Dans la négative, l'interlocuteur répondait en donnant le nom du lieu où il avait été réellement circoncis à quinze ans. Le terme karimania fut inventé pour la circonstance, et J. Kariuki confesse qu'il ne l'avait jamais entendu prononcer auparavant. Il pourrait se traduire par * tourner et retourner la terre ${ }^{83}$. Ce mot de passe est doublement révélateur :

a) La question : «ù as-tu été circoncis? », indique que le prêt du serment est une nouvelle initiation. L'emprunt d'éléments à l'appa. reil rituel de l'initiation traditionnelle le confirme. Ainsi, les adolescents devaient passer sous une arche faite de tiges de bananier, de canne à sucre. L'officiant qui circoncisait avait le visage enduit de craie $(i r a)^{\text {st }}$ et aspergeait les novices de miel et de lait. Ces derniers devaient eux-mêmes prêter un serment : " Ils jurent qu’à partir de ce jour ils se conduiront comme des adultes et sauront prendre leurs responsabilités afin de contribuer au bien-être de la communauté et qu'ils ne reculeront pas devant les devoirs exigés pour sa protection ou son développement. En outre, ils promettent de ne jamais révéler les secrets de la tribu même à un de ses membres, s'il n'a pas été initié ${ }^{\star s}$. Le choix de ces éléments par les leaders de la K.C.A. renseigne donc sur les objectifs et les intentions du mouvement : raffermir l'unité de la tribu, frapper l'imagination des postulants en leur rappelant une cérémonie de passage et d'intégra-

83. J. M. KarIUKI, Mau-Maí Detainee..., op. cit., 1963, p. 28.

84. Symbolisant la coucbe de Ngaĩ (divinité suprême des Kikuyu, résidant sur le mont Kenya, montagne de la blancheur $\$$ ).

85. J. KanyatTA, op. cit., 1967, p. 103. C'est nous qui soulignons. 
tion qui, en même temps qu'elle leur donnait statut et droit, leur inculquait une discipline sociale et des devoirs.

b) La réponse : « J'ai été circoncis à Karimania par Karimania », fait intervenir la terre comme élément de reconnaissance et d'unité et renvoie à une autre catégorie de cérémonies traditionnelles, celles qui avaient lieu lors des échanges économiques ou lors du bornage des terres, où le prêt du serment était monnaie courante : \&e vendeur et l'acquéreur prêtent alors serment devant les anciens. L'acquéreur offre un bélier qui est égorgé sur les lieux où sont conclus les accords [...] Puis, [sur] la limite du champ que lui désigne le propriétaire, l'ancien de cérémonie répand, en marchant, les entrailles du bélier [...] L'opération achevée [...], le vendeur et l'acquéreur entourent chacun leur poignet droit d'une de ces peaux ${ }^{88}$. Par cet acte qui termine la cérémonie du bornage, les deux hommes se considèrent parents par alliance ${ }^{87}$. >

On doit cependant rechercher les origines rituelles du serment de l'unité dans le système juridique traditionnel, où il constituait l'élément essentiel de la procédure. Les deux parties en présence, plaignant et offenseur, de même que les juges, devaient prêter un serment. Cette prestation garantissait les témoignages et évitait les corruptions. Il existait trois formes de serment, ordonnées selon la gravité de la faute, et \& nul n'oserait prêter l'un d'eux s'il n'est persuadé de son innocence et de la justesse de sa cause ${ }^{88}$ \.

Le premier (muma), utilisé lors de petits litiges, se déroulait de la manière suivante : un agneau était immolé; le contenu de ses entrailles était mélangé à de l'herbe, de l'eau et du sang de l'animal. Le tout était placé dans un trou creusé dans la terre. Le devin (mondo ogo) y plongeait de petites baguettes de bois qu'il faisait ensuite lécher au plaignant et à l'offenseur, ces derniers devant ensuite prêter le serment suivant :

* Si je mens, que ce symbole de vérité me tue!

$\mathrm{Si}$ j'accuse quelqu'un faussement, que ce symbole de vérité me tue !

Si ce que je réclame ne m'appartient pas, que ce symbole de vérité me tue!

Le second serment (koumi) intervenait dans les procès qui met-

86. Peau du bélier sacrifié.

87. J. KENYATTA, op. cit., 1967, p. 45 et 46.

88. Ibid., p. 154 . 
taient en cause plusieurs personnes. Tous les membres du kiama (conseil) prêtaient serment en cassant les membres d'un jeune bouc qui était ensuite transporté dans un champ désert et inculte, où ils le frappaient avec une pierre rituelle (ugangae) en disant :

\&Que mes os soient brisés comme ceux de ce bouc si la propriété que je réclame n'est pas la mienne!

Que ma famille soit brisée comme les os de ce bouc si je réclame plus que mon droit ! 》

Le troisième serment ne se prêtait que dans des cas graves : vols, crimes, sorcellerie. Le gethatie (tel était son nom) était symbolisé par une petite pierre rouge percée de sept trous ${ }^{89}$, qui étaient censés représenter les sept orifices du corps humain. Les accusés devaient faire passer une brindille sept fois de suite dans chacun des sept orifices en jurant de dire la vérité.

Le serment de l'unité, en syncrétisant les rituels propres à des cérémonies traditionnelles clés (initiation, bornage, procédures), résumait en quelque sorte l'appareil rituel de la société kikuyu. Comme dans les mythes d'origine ${ }^{90}$, le bétail, et notamment la chèvre, apparaît comme le médiateur entre l'ordre divin et l'ordre social, comme le symbole de l'alliance, et réaffirme symboliquement la naissance des enfants de Gikuyu et Mumbi, ici l'Unité Gikuyu et Mumbi. La motte de terre humide, pressée sur le nombril ou sur l'estomac, rappelle l'origine chtonienne des hommes et dévoile les liens quasi sexuels avec la terre. Les boyaux qui enchaînent le postulant symbolisent le cordon ombilical et rappellent le rite de la seconde naissance (cf. supra). Ainsi, la forte charge symbolique et magique des éléments rituels du serment de l'unité, de même que les menaces physiques ou surnaturelles généralement proférées pour prévenir le parjure et la déviance avaient pour but de lier et d'engager totalement l'individu dans l'organisation, de s'en assurer enfin.

Cependant, l'utilisation d'encoches rituelles traditionnelles dans la prestation du serment de l'unité était moins un retour à la tradition ou un calque de celle-ci, comme certains observateurs l'ont cru $^{\text {91, }}$, qu'une recomposition ou une redéfinition culturelles élaborées à partir de la systématique des représentations, en fonction d'une situation « moderne» et suivant une perspective politique qui se tra-

89. Qui rappelle le ngata, utilisé principalement dans le serment batuni. 90. Cf. J. JAMIN, Contacts et antagonismes culturels..., op. cit., 1973, p. 48-50.

91. Cf. W. E. MŬHLMANN, Messianismes révolutionnaires..., op. cit., 1968. 
duisait notamment dans le commentaire politique qui terminait chaque prestation. Ces cérémonies manifestaient en tout cas une certaine « insolence » à l'égard de la tradition, puisque les éléments et les valeurs retenus en étaient mélangés, triturés, grossis et amplifiés par des officiants souvent peu officiels! C'est dire en somme que, au lieu de sauvegarder ou de restaurer le temps et l'espace de la tradition, les prestations de serments, tout en utilisant le même stock de signifiants, finissaient par marginaliser les participants, par en faire une «bande à part » et par créer un mode de solidarité et d'organisation nouveaux en les plaçant aux frontières de l'interdit, de la souillure et de l'impureté (notamment par la citation rituelle du serment gethatie qui se prêtait en cas de sorcellerie). De ce point de vue, les thèses de la régression, de l'atavisme ou du réarmement idéologique souvent avancées par la littérature officielle de l'époque pour définir ces cérémonies paraissent peu convaincantes, d'autant que ces cérémonies, bien qu'énumérant rapidement les signifiants phares de la culture traditionnelle, en laissaient de côté et non des moindres : il en est ainsi de la communion avec les ancêtres. $R$. Buijtenhuijs montre très bien que cet oubli peut être intentionnel et répondre à des exigences stratégiques : « $\mathrm{La}$ communion avec les ancêtres, toujours des ancêtres familiaux, ne pouvait que gêner les desseins à long terme des dirigeants Mau-Mau, dans la mesure où le mouvement Mau-Mau se voulait consciemment un mouvement d'unification du peuple kikuyu tout entier. Loin de favoriser l'intégration nationale, la communion avec les ancêtres risquait d'introduire au sein du mouvement un élément de particularisme. Par contre, les ancêtres mythiques tribaux Gikuyu et Mumbi faisaient partie intégrante de l'ensemble des symboles Mau-Mau : consciemment ou inconsciemment, les idéologues Mau-Mau semblent avoir voulu faire sauter les cadres sociaux et spirituels trop étroits de l'ancienne civilisation kikuyu et transposer la communion avec les ancêtres du plan de la famille au plan national où elle pouvait jouer un rôle positif et dynamique très important ${ }^{\circ 2}$. 》

Cela tendrait à prouver que les prestations du serment, loin d'être une simple « mobilisation de l'arsenal spirituel et religieux kikuyu », visaient d'autres objectifs - ceux-là plus politiques - et s'acharnaient à casser l'organisation traditionnelle qui était d'une certaine manière compromise par la collaboration tacite de ses chefs avec

92. R. BUIJTENhUIJS, op. cit., 1969, p. 289. 
l'administration coloniale. L'oubli du culte des ancêtres est en effet de taille, et par là même significatif, puisque ce culte conditionnait et orientait la problématique foncière ${ }^{93}$ et la politique lignagère (cf. supra, le rôle des ukuru).

Cet oubli, et d'une façon générale cette déviation, voire cette perversion de la culture traditionnelle peuvent en outre s'expliquer par le fait que les organisateurs des prestations du serment de l'unité étaient surtout des Kikuyu squatterisés. Expulsés des terres du Kiambu par les colons européens ou des réserves du Fort-Hall et du Nyéri par la pression démographique et par la détérioration des terres cultivables, les squatters formaient la majorité des travailleurs agricoles. Lié par un contrat qu'il « signait en présence d'un représentant du gouvernement colonial, le squatter * s'engageait à donner, lui et sa famille - chacun des membres mâles âgés de plus de seize ans -, 180 jours de travail par an, payables tous les 30 jours, selon le salaire que le propriétaire et l'indigène fixaient d'un commun accord ${ }^{*}$ ». Le contrat était d'une durée minimum de trois ans et le salaire mensuel variait de 10 à 18 shillings. Le squatter habitait avec sa famille sur la plantation où le colon lui abandonnait deux acres de terre. Il était autorisé à avoir quinze têtes de bétail (exclusivement chèvres et moutons); il lui était interdit de couper des arbres et de défricher. « Toutes les femmes et tous les enfants devront travailler à la requête du propriétaire ou du planteur. Etre continuellement en retard ou absent peut signifier un renvoi immédiat et la perte de la shamba (case et lopin de terre du squatter) ${ }^{95}$. $\gg$ Ce système avait donc l'avantage de fournir au colon une main-d'œuvre permanente. Quant au squatter, s'il voyair sa sécurité économique garantie, il finissait par perdre tout contact et tout lien avec son ethnie : il était detribalized.

Paysans déracinés, d'une certaine manière prolétarisés, coupés du monde blanc par la color-bar, éloignés de la tribu par les limites de la plantation ${ }^{\circ}$, en fait socialement expatriés, certains squatters

93. Toute transaction foncière et toute opération culturale nécessitaient l'intervention symbolique des ancêtres familiaux, par l'intermédiaire des ukuru.

94. M. SAlVAdORI, La Colonisation européenne au Kenya, Larose, Paris, 1938, p. 183.

95. F. BrockwaY, « Pourquoi y a-t-il des Mau-Mau? \, Esprit, n ${ }^{\circ} 205$, Paris, 1953, p. 258.

96. L'institution du livret de travail (kipande) permettait aux colons comme à l'administration de contrôler efficacement les allées et venues des 
- notamment ceux qui avaient été expulsés des terres du Kiambu tentèrent donc sur un mode magique, par le serment de l'unité et dans le cadre de la Kikuyu Central Association, de retrouver et de redéfinir une position culturelle. Ils essayèrent par là de se démarquer par rapport aux loyalistes (collaborateurs) et aux chefs coutumiers (nommés par l'administration coloniale), suspectés d'avoir négocié à leur profit les terres du Kiambu, et de se remarquer, par \& une parodie \ initiatique, dans un même champ idéologique mais avec des règles syntaxiques différentes. L'emprunt privilégié et insistant d'éléments de l'appareil initiatique traditionnel traduit une volonté de différenciation en même temps qu'une recherche d'identité.

En définitive, l'utilisation du serment dans une perspective d'unification culturelle et politique avait comme caractéristique et incidence majeures d'exiger de l'individu un engagement total qui transcendait ses autres obligations. Le serment définissait les « amis $\gg$ et les \& ennemis \$, traçait les frontières du mouvement et contribuait dans une certaine mesure à intensifier les conflits entre les Kikuyu et les Européens, entre les membres de la Kikuyu Central Association et les loyalistes. L'hostilité et la répulsion de ces derniers devant ces cérémonies encouragèrent certainement, par réaction, les leaders du mouvement à y recourir systématiquement et à renchérir sur leurs rituels, accusant par là une volonté de préservation et de différenciation. Cela fut même, devant la violence de la répression coloniale et face aux risques de démantèlement de la Land Freedom Army, poussé à l'extrême. Certains leaders et certains groupes ${ }^{97}$ n'hésitèrent pas à utiliser le cadre initiatique du serment pour pervertir la morale traditionnelle, en plaçant précisément les individus dont on exigeait le silence et la soumission dans une situation de souillure et d'impureté rituelles (thabu). Le serment batuni qui apparut dans ce contexte transgressait les interdits fondamentaux en organisant des orgies, en utilisant du sang menstruel et des cadavres humains, etc., et créait de la sorte des « contre-valeurs s dont le but final était de o faire des combattants Mau-Mau de véritables desesperados engagés à $100 \%$ dans la révolte et prêts à commettre n'importe quel crime. Par la rupture de tous les tabous inviolables,

squatters, leur histoire personnelle, leurs relations, leur caractère et leurs - idées politiques $\gg$, puisque tous ces éléments devaient y être consignés par les différents employeurs.

97. R. Buijtenhuijs a raison de souligner le caractère accidentel et limité de ces pratiques. 
le serment avancé ${ }^{98}$ aurait été une véritable " mise en condition" des combattants destinée à briser et à anesthésier la conscience, un véritable " doping "pour leur permettre de supporter la tenision psychologique qui découlait de la guerre Mau-Mau avec ses actes de violence et de terreur contre les populations civiles kikuyu et européennes $^{99} \gg$. Sans faire trop de cas de cette pathologie rituelle qui resta, malgré la publicité qui en fut faite par la presse de l'époque, limitée et somme toute marginale, il faut reconnaître que le serment constituait un puissant moyen de contrôle et de recrutement, à tel point d'ailleurs que les forces de l'ordre instituèrent, avec l'aide des loyalistes et des chefs coutumiers, des contre-serments pour « libérer la parole » de certains « envoûtés », pour les dégager de l'organisation et pour les replacer dans le cadre rassurant de la tradition. Cette guerre rituelle fut cependant un échec pour l'administration coloniale. E. Bustin en faisait la remarque : «Un inconvénient cependant demeure : c'est que ces cérémonies [les contre-serments], respectant les coutumes de la tribu, se déroulent en plein jour... et en public, ce qui exige donc des indigènes sincèrement désireux d'être absous un courage et une fermeté assez rares. Les conjurés réagissent d'ailleurs immédiatement, soit en procédant pour l'exemple à quelques exécutions, soit en neutralisant l'effet de ces cérémonies par de spectaculaires contre-exorcismes. Il existe surtout un autre inconvénient aux rites de purification : c'est leur totale incapacité à prévenir le renouvellement ultérieur du serment annulé. Ainsi des villages entiers montrent-ils un égal empressement pour abjurer le Mau-Mau et pour s'y faire à nouveau initier quelques jours plus tard. Le bilan net des cérémonies lustrales demeure donc assez maigre en dépit des espoirs que cette pratique avait suscités et bientôt le gouvernement devra renoncer à leur usage ${ }^{100}$. > R. Buijtenhuijs note également que, "par rapport à cette cérémonie " chaude ", combien ont dû paraître froids et plats les rites de purification du gouvernement, rites qui n'initiaient à tien, sinon à la trahison, et qui ne faisaient appel à aucun sentiment élevé. A la renaissance spirituelle Mau-Mau, le gouvernement colonial n'a su opposer qu'une technique relevant du calcul, technique en quelque sorte périmée,

98. Autre dénomination du serment batuni, surtout en vigueur chez les auteurs anglo-saxons.

99. R. BuIJTENHUIJs, op. cit., 1969, p. 303.

100. E. BUSTIN, La Décentralisation administrative et l'évolution des structures politiques en Afrique orientale britannique, Liège, 1958, p. 381. 
pour la raison même quielle respectait la tradition sur tous les points ${ }^{101}$ 》. Cet échec des contre-serments traduit en effet une erreur d'appréciation et d'interprétation de la part des autorités coutumières et coloniales, car c'étaient moins les contenus que les règles idéologiques de juxtaposition et d'accord qui caractérisaient les serments Mau-Mau et qui définissaient leur écart à la norme et à la coutume. Le secret demandé n'avait donc pas pour fonction de dissimuler le contenu des cérémonies, mais visait plutôt à prendre et à reprendre la parole des postulants dans de nouvelles règles syntaxiques.

Pas plus ici que dans les initiations classiques, le secret imposé n'occulte un savoir précis et constitué. Les vœux exprimés ne dévoilent ni ne mentionnent une stratégie militaire. Le commentaire final reste succinct. Les participants sont laissés dans l'ignorance des processus de décision et sont souvent « enrôlés 》 de force ${ }^{102}$. Le corpus des vœux possède une coloration nettement négative : il s'agit d'exprimer et de transmettre des interdits, ne pas dire, ne pas faire - ce qui accuse en somme l'aspect répressif de la cérémonie qui déplace sans arrêt la menace sur un registre symbolique (le sang, la

101. R. BuijtenhuiJs, op. cit., 1969, p. 295.

102. Comme en témoigne ce récit de J.M. Kariuki : * Un soir de février 1950, je décidai de rentrer au village pour voir ma fiancée. Lorsque j'arrivai à la case vers 19 h 30 , elle n'était pas là mais je trouvai des gens du village qui étaient assis, discutant, riant, racontant des histoires. La curiosité me fit rester. Je voulais savoir quel genre de réunion c'était et je me joignis à la conversation. Vers 21 heures, un homme entra, dit qu'il cherchait des jeunes hommes vigoureux pour l'aider dans un travail. Trois d'entre nous se portèrent volontaires et nous suivîmes l'homme dans l'obscurité. Sa case n'était qu'à quelques mètres devant. A l'intérieur, je vis une quinzaine de personnes. Mon père était là et aidait d'autres hommes à sacrifier un agneau. Dans le courant de la conversation, je demandai à un homme ce qui se passait et il me dit que tout le monde attendait la visite d'un personnage important [...] Après quelques minutes, on me demanda de sortir et, avec six autres, je fus conduit vers une case située en dessous d'acacias et séparée de notre maison par une haie. Les gens qui nous accompagnaient agirent comme s'ils étaient des gardes - ce qu'en fait ils étaient. Comme nous approchions de la porte, je vis une lueur blafarde à l'intérieur et j'entendis des gens chuchoter. Mais quand nous entrâmes, la lueur disparut et il $y$ eut un silence total. Nous étions tous effrayés et nous entrâmes avec répugnance, poussés par les gardes. A l'intérieur, il faisait noir comme dans un four, mais je pouvais entendre le murmure de voix qui semblaient poser des questions sur nous. Je me rappelle avec douleur quelques instants de terreur pendant lesquels nous fûmes immobilisés par trois ou quatre individus qui nous emprisonnaient les bras et nous serraient la gorge. Un moment plus tard, cependant, quelqu'un ordonna d'allumer et bientôt trois lampes tempête éclairèrent l'intérieur de la case $\diamond(0 p$. cit., p. 25). Suit la description du serment proprement dit. 
terre, etc.). Le secret dans ce cas a une valeur essentiellement formelle, distanciatrice, voire aliénante, pour ceux qui sont soumis à sa loi, non pas parce qu'il garde ou retient des informations, mais parce qu'il les place d'emblée dans une problématique éthique : se taire ou trahir. 


\section{CONCLUSION}

Pour certains, et pour P. Clastres en particulier, la société \& primitive \'est pas divisée : elle ignore l'autorité, l'oppression, l'Etat. Il n'y a rien, écrit P. Clastres, dans le fonctionnement économique d'une société primitive, d'une société sans Etat, rien qui permette l'introduction de la différence entre plus riches et plus pauvres, car personne n'y éprouve le désir baroque de faire, posséder, paraître plus que son voisin. La capacité, égale chez tous, de satisfaire les besoins matériels et l'échange des biens et services, qui empéchent constamment l'accumulation privée des biens, rendent tout simplement impossible l'éclosion d'un tel désir, désir de possession qui est en fait désir de pouvoir. La société primitive, première société d'abondance, ne laisse aucune place au désir de surabondance ${ }^{1}$. \& L'extension logique et géographique donnée ici à cette notion de société primitive est abusive et contestable, mais traduit une démarche courante en anthropologie; démarche qui, à partir d'une certaine cohérence des discours, d'une unité et d'une harmonie idéologiques, postule une homogénéité sociale, sans que soit envisagé ce que ces discours taisent. La façon dont $\mathrm{P}$. Clastres décrit, ou plutôt imagine, la société primitive caractérise peut-être celles

1. P. Clastres, op. cit., 1974, p. 174 . 
qui lui ont servi de références (les sociétés amérindiennes), mais ne s'accorde pas en tout cas à la réalité socio-économique des sociétés «primitives $\gg$ ou « sans Etat » africaines. Le fait que pour celles-ci la richesse ne se montre pas, ne se dise pas, ne signifie en rien qu'elle n'existe pas, qu'il n'y ait pas des mécanismes d'accumulation, de thésaurisation, de confiscation; qu'il n'y ait pas un désir «baroque de posséder plus que son voisin ». Il en est de la richesse comme du savoir : elle doit rester secrète sous peine d'être contestée et de rentrer dans une problématique de l'agression imaginaire (sorcellerie), d'autant que sa destination (ou sa conversion) demeure principalement symbolique et culturelle, et son utilisation individuelle.

Les funérailles qui, paradoxalement, sanctionnent la vie des Sénoufo sont une mise en sène sociale, brutale et fugitive, de l'inégalité économique : elles montrent et annulent dans un même temps une partie du capital acquis par le défunt ${ }^{2}$, comme elles montrent et annulent aussi celui des apparentés dont la contribution est théoriquement fonction de leur richesse. Il ne s'agit pas, contrairement à une opinion largement répandue ${ }^{3}$, d'une égalisation sociale des richesses, puisque, d'une part, celles-ci ne sont pas redistribuées mais sont détruites et consommées immédiatement suivant l'apport initial ${ }^{4}$, et puisque, d'autre part, leurs moyens de production restent inaliénables, non sécables, non distribuables. Par ce « gaspillage » ostentatoire de richesses, les funérailles sénoufo masquent d'une certaine façon les rapports de forces et d'exploitation qui conditionnent leur production et qu'elles tendent à reproduire, tout en paraissant les annihiler ou les amortir. Car si la dette "funéraire 》 aplanit dans un temps raccourci, celui des fêtes, les inégalités socio-économiques, elle en affirme et en justifie l'existence par sa démonstration. La représentation de la mort et le rituel funéraire chez les Sénoufo semblent favoriser des comportements économiques d'accumulation, ne serait-ce que par l'acquisition des pagnes funéraires, secrètement conservés dans une malle ou une valise, dont l'importance et la valeur varient suivant la position sociale du détenteur, dépendent donc de son pouvoir de disposer à des fins personnelles du surpro-

2. Notamment lors de la cérémonie de présentation et de remise des pagnes funéraires.

3. Cf. L. ROUSSEL, op. cit., 1965, p. 77.

4. Ce qui revient à dire, comme on me l'a fait remarquer, que \& les meilleurs morceaux sont donnés à ceux qui ont donné le plus ì. 
duit. La cérémonie de remise des pagnes, cérémonie centrale et publique dans le rituel funéraire sénoufo, dévoile et confirme ce pouvoir du défunt, accrédite d'une certaine manière sa position dans l'ordre lignager et ancestral. Une partie variable du stock de pagnes, offerts ou accumulés, est « détruite » en l'enterrant avec le défunt, l'autre revient à l'héritier direct et s'additionne à son stock propre. Cette inégalité dans la mort traduit une inégalité dans la vie.

D'une façon voulue plus sérieuse (notamment par l'arsenal formel et conceptuel qu'elle utilise et qui paraît la garantir), la démarche ethnoscientifique américaine n'évite pas, tant dans son approche que dans ses méthodes, le postulat de l'homogénéité et de l'indifférenciation sociales des sociétés traditionnelles, déduites de celui de l'unité culturelle et de la « cohérence des savoirs ». Se proposant d'étudier * comment chacun forge son champ d'expérience à partir de la façon dont il en parle ${ }^{5}$ », elle postule l' « équivalence cognitive $\gg$ des informateurs ${ }^{6}$. Chacun est vu comme le dépositaire privilégié, comme l'utilisateur moyen du code culturel. Une telle démarche, outre qu'elle révèle la \& pesanteur linguistique », suppose une conception non différentielle du contenu de l'éducation, nie ou néglige les faits de sélection, de manipulation, de confiscation des codes symboliques. Faute d'envisager la dynamique du phénomène classificatoire à des niveaux même formels, et faute de poser le problème des relations entre structures de codification, de communication et de subordination, elle se condamne à découper la réalité socio-culturelle en « domaines $\triangleright$ et à voir dans la culture une simple juxtaposition de taxonomies. Par sa vocation et sa tentation descriptives, l'ethnoscience a sans doute permis de raffiner sur les méthodes de collecte et de traitement des données culturelles (d'où son nom de new etbnography), mais son apport risque d'être limité et même vain si elle ne tente pas d'analyser en même temps \& la structure du système des relations sociales où s'engendrent ces systèmes cultu-

5. C.O. FraKB, \& The Ethnographic Study of Cognitive Systems $\gg$, in T. Gladwin et W. C. Sturtevant, eds., Antbropology and Human Bebavior, Washington, 1962 , p. $73-85$.

6. Cela est d'ailleurs caricatural dans la thèse de M. BlACK, An Etbnoscience Investigation of Ojibwa Ontology and World View, Stanford University, 1967, où elle remarque qu'il lui aurait fallu une très longue période d'observation pour s'approcher au plus près de ce que c'est que d'être un Indien Ojibwa, que ne le permirent les dix mois de travail intensif avec une poignée d'informateurs dans une chambre blanche, derrière un bureau. 
rels ou symboliques et où se définissent les fonctions sociales qu'ils templissent à un moment donné du temps ${ }^{7}$, .

L'exemple des tendeurs ardennais tend justement à montrer comment un savoir, certes précis et limité (que l'on peut toutefois supposer a priori partagé par l'ensemble du personnel technique), se trouve pris dans un réseau social qui en conditionne l'acquisition, l'utilisation et la transmission, qui pondère donc sa neutralité et sa \& majuscule \&, qui délimite enfin ses zones de partage. C'est au niveau de son expression qu'interviennent ici des phénomènes de censure, de rétention, voire de répression, redoublant ou soulignant des rapports de pouvoir, inscrivant des statuts et manifestant des processus d'accumulation et de confiscation.

Même dans les sociétés lignagères où les instances pédagogiques paraissent plus diffuses, moins différenciées, le savoir 》 ne se distribue pas d'une façon homogène. L'oralité n'est pas forcément source d'égalité. Le secret ou le silence introduisent des ratés dans la communication sociale, endiguent la transmission des savoirs, expriment des coupures et des retraits qui subordonnent et divisent. Qu'il soit donc de l'ordre du procédé ou de la supercherie, de l'ordre de la tactique ou de la stratégie, ou encore de celui de la confiscation, le secret a une fonction distanciatrice et une valeur hiérarchique. En maintenant ou en affirmant des zones d'ombre ou d'incertitude, il démultiplie les lieux sociaux de reproduction culturelle, soit en réservant certains savoirs à certaines catégories sociales, soit en censurant leur expression.

La réalité sociale et culturelle des sociétés lignagères (pour ne parler que d'elles) n'a pas cette transparence que lui confèrent les discours ou les théories locales. Celles-ci ne sont pas à prendre au mot, ainsi que le suggèrent ces remarques de $M$. Augé : « Le mot prononcé, dans son contexte, se charge du sens des mots passés sous silence, dont il constitue une manière d'équivalent métaphorique; c'est parce qu'il est des mots qu'on n'emploie pas (hors les cas de crise) que tout peut être signifié; la loi du silence. fait l'art de la parole; la prudence impose le recours aux détours métaphoriques et métonymiques; la prudence mais aussi le sens de l'efficacité ; nul discours n'est mieux entendu que celui qui n'est pas tenu, qui double les propos effectivement tenus, symbole absent d'une complicité

7. C. FoURNIER, A propos de l'ethnoscience $\$$, Revue frangaise de sociologie, vol. XII, n' 4, Paris, 1971, p. $459-492$. 
tacite entre partenaires d'une relation non avouée". Q Que ces discours occultent ou masquent les contradictions, les rapports de forces et de subordination, cela ne fait aucun doute, mais ils en sont également, par ce qu'ils taisent et du fait même qu'ils taisent, la description. De ce point de vue, rien n'est plus troublant que le discours et la symbolique initiatiques. Le grossissement et l'interprétation de celle-ci, généralement fondée sur la « conjuration $\gg$ de la disjonction naissance/mort, ont en fait de quoi surprendre, même s'ils sont très souvent pàrtagés et affirmés. Ce n'est d'ailleurs pas tant leur universalité qui surprend que leur insistance et transparence. Il est presque toujours dit que les rituels initiatiques manifestent symboliquement une naissance sociale en confirmant la mort du \& biologique \$, en d'autres termes informent socialement et culturellement le biologique, font des enfants dépendants des adultes responsables, des êtres sociaux... Je caricature à peine ce qui ressort de thèses, souvent brillantes, sur l'initiation. Un ouvrage récent, théoriquement averti de celles-ci, s'en fait l'écho et affirme sans ambages que * l'enfant non initié n'a fait que naître biologiquement, il n'a encore qu'un père et une mère " réels" ; pour devenir un être social, il lui faut passer par l'événement symbolique de la naissance/mort initiatique, il lui faut avoir fait le tour de la vie et de la mort pour entrer dans la réalité symbolique de l'échange ${ }^{*}$. C Cest là méconnaître la dimension sociologique et symbolique de la petite enfance, de la préadolescence et de la préinitiation, certes peu étudiées mais qui paraissent, au regard de certains travaux, littéralement envahies par l'apprentissage des échanges et des différenciations, et souvent bousculées par des interrogations, des diagnostics et des définitions : « L'enfant qui naît est certes perçu et conçu comme un être inachevé, mais aussi et surtour comme un étranger dont le comportement sera modulé et " apprivoisé " à la suite d'interrogations multiples qui tentent de préciser son origine et son identité. Le corps de l'enfant, parce qu'il est conçu comme étranger, voire étrange, est ce lieu privilégié où se manifeste, se discute et s'expérimente le système de représentation de la personne : les identifications proposées et les hypothèses formulées à son sujet peuvent en dévoiler l'articulation et la systématique. La recherche de l'origine et

8. M. Augt, op. cit., 1975, p. 408.

9. J. BAUdRILlaRD, L'Ecbange symbolique et la mort, Gallimard, Paris, 1976, p. 203. 
la constitution de l'identité paraissent orienter et conditionner la mise en ouvre de l'appareil de socialisation ${ }^{10}$.

On peut dès lors s'interroger sur l'opportunité et le sens d'un discours et d'une symbolique, celui et celle de l'initiation, qui, en affirmant la naissance sociale des néophytes par des rites de mortification, par des simulacres de mort biologique, paraissent nier dans un même temps les efforts précédemment déployés pour situer et éduquer l'enfant, pour indiquer et définir ses itinéraires sociaux, comme si l'ordre social postinitiatique devait être différent... Dans cette négation parfois brutale des antécédents et du passé social des néophytes ${ }^{11}$, il me paraît $\mathrm{y}$ avoir bien plus qu'un simple marquage symbolique de la socialisation. En jouant en effet sur des couples de termes naturellement irréductibles tels que homme/femme, adulte/enfant, le discours initiatique tend à naturaliser les rapports sociaux, à faire basculer l'ordre social dans l'ordre naturel - et cela au moment précis où ils s'articulent l'un à l'autre (puberté) -, à justifier l'un par l'autre, à dissimuler enfin les fondements et articulations de l'un par la couverture écologique de l'autre. Ici, comme pour la « parole du pouvoir \$ (cf. supra, chap. 2), il s'agit de décentrer les lignes de force, de transformer les rapports de forces en rapports de sens... de discipliner naturellement le social, car nulle discipline n'est mieux comprise et plus efficace que celle qui se prétend et se veut naturelle.

Le discours initiatique est donc à la fois métaphorique et métonymique : il parle des adultes pour dire les aînés et pour s'adresser aux cadets; il parle du biologique pour dire le social. Il fonctionne selon une loi du silence qui, sous la forme de la « mise au secret \$ des futurs initiés, tait pour un temps leurs origines et leur identité sociales, qui, sous la forme du « secret imposé 》, maintient vis-à-vis des autres, des cadets; la distance, l'ambiguïré et l'incertitude de la naissance et donc des itinéraires. C'est au prix de ce mutisme et de ce silence que se fonde, se produit et se reproduit ce que M. Augé appelle l' * unicité idéologique $\gg$ des sociétés lignagères. Puisant dans le matériau biologique (mort/naissance) pour signifier un

10. J. RABaIn, Le Corps, la parole et l'objet. Stratégies de l'apprentissage social chez les Wolof du Sénégal (à paraître).

11. Nous avons vu (cf. chap. 3, section III) que les rites d'initiation se caractérisent souvent par la perte du nom, du passé et des liens de parenté des futurs initiés, qui sont de la sorte réduits à l'état de chose informe et innommable $\$$. 
passage, un changement, l'appareil initiatique permet d'offrir à chacun une nouvelle naissance qui redistribue les cartes et désamorce le poids des origines... du moins le fait croire. De ce fait, les « contre-idéologies 》 ne sont ni réprimées ni censurées; elles n'ont pas lieu d'être, elles sont inutiles, puisque chacun en devenant adulte deviendra aîné. Ici est toute la ruse de la raison initiatique : faire croire que l'ordre social est un ordre naturel. 


\section{OUVRAGES CITÉS}

ADLER A. et ZBMPLENI A., Le Báton de laveugle. Divination, maladie et pouvoir cbez les Moundang du Tcbad, Hermann, Savoir, Paris, 1972, $223 \mathrm{p}$.

AUGÉ M., éd., La Construction du monde. Religion, représentations, idéologies, Maspero, Dossiers africains, Paris, 1974, $136 \mathrm{p}$.

AUGÊ M., Théorie des pouvoirs et idéologie. Etude de cas en Cóte-d'Ivoire, Hermann, Savoir, Paris, 1975, 440 p.

Balandier G., Antbropologie politique, P.U.F., Le sociologue, Paris, 1967, $240 \mathrm{p}$.

BAREL Y., La Reproduction sociale. Systemes vivants, invariance et cbangements, Anthropos, Paris, 1973, 558 p.

Batzson G., La Cérémonie du naven, éd. de Minuit, Le sens commun, Paris, 1971, 311 p.

Baudrullard J., Pour une critique de l'économie politique du signe, Gallimard, Les essais, Paris, 1972, 270 p.

BETTElheim B., Les Blessures symboliques, Gallimard, Connaissance de l'inconscient, Paris, 1971, 252 p.

Bocher G., « Le Poro des Diéli s, Bulletin de l'L.F.A.N., série B, t. XXI (1-2), Dakar, 1959, p. 61-102.

Bochet G., c Les Masques sénoufo, de la forme à la signification >, Bullotin de l'l.F.A.N., série B, t. XXVII (3-4), Dakar, 1965, p. 636-677.

BOURdieu P., Esquisse d'une tbéorie de la pratique, Droz, Paris, 1972, 269 p.

BourdieU P. et PASSERon J.C., La Reproduction. Eléments pour une theorie du système d'enseignement, éd. de Minuit, Le sens commun, Paris, 1970, 281 p.

BuIjtanhuljs R., Le Mouvement Mau-Mau. Une révolie paysanne et anti- 
coloniale en Afrique noire, thèse de $3^{\circ}$ cycle, E.P.H.E., VI section, Paris, 1969, 523 p., éditions Mouton, Paris, 1971.

Cagnolo C., The Akikuyu. Their Customs, Traditions and Folklore, Mission Printing School, Nyeri, 1933, 324 p.

CAstel R., Le Psycbanalysme, Maspero, Textes à l'appui/Psychiatrie, Paris, 1973,281 p.

Cazeneuvb J., Les Rites et la Condition bumaine, P.U.F., Paris, 1958, 497 p.

CAzIn F.-S., « Mémoires. La vie à Rocroi sous le Premier empire $\star$, Présence ardennaise, $\mathrm{n}^{\circ} 17$, Mézières, 1954, p. 4-20.

Clastres P., La Société contre l'Etat, éd. de Minuit, Critique, Paris, 1974, $186 \mathrm{p}$.

ClEMENT C.B., Le Pouvoir des mots. Symbolique et idéologique, Mame, Repères, Paris, 1973, 173 p.

Deleuze G. et GuatTARI F., L'Anti-CEdipe. Capitalisme et scbizopbrénie, éd. de Minuit, Critique, Paris, 1972, 470 p.

Ducrot 0 ., Dire et ne pas dire... Principes de sémantique linguistique, Hermann, Savoir, Paris, 1972, 283 p.

Estrvant A., \& Les Tenderies aux grives en Ardennes \$, Bulletin des fédérations des chasseurs de la Moselle, 1962, $\mathrm{n}^{\circ} 62$, p. 27-29, et $\mathrm{n}^{\circ} 63$, p. 13-15.

ETIBNne P., Les Interdictions de mariage chez les Baoulé, Centre O.R.S.T.O.M. de Petit-Bassam, Abidjan, 1972, 135 p.

Foucault M., Surveiller et punir. Naissance de la prison, Gallimard, Bibliothèque des histoires, Paris, $1975,318 \mathrm{p}$.

François M., \& Le Tendeur et les Saisons \$, Etudes ardennaises, $n^{\circ} 32$, Mézières, 1963, p. 3-9.

Frankitn J., Le Discours du pouvoir, U.G.E., 10/18, Paris, 1975, 429 p.

GIRard R., La Violence et le Sacré, Grasset, Paris, 1972, 451 p.

GofFMAN E., Asiles. Etudes sur la condition sociale des malades mentaux, éd. de Minuit, Le sens commun, Paris, 1968, 447 p.

Gofpman E., Les Rites d'interaction, éd. de Minuit, Le sens commun, Paris, $1974,230 \mathrm{p}$.

HOBLEY C. W., Bantu Beliefs and Magic, with Particular Reference to the Kikuyu and Kamba Tribes of Kenya, Witherby, Londres, 1938, 374 p.

HOLAS B., Les Sénoufo (y compris les Minianka), P.U.F., Monographies ethnologiques africaines, Paris, 1966, $183 \mathrm{p}$.

Holas B., Les Dieux d'Afrique noire, P. Geuthner, Paris, 1968, 284 p.

Jamin J., La Nébuleuse du Koulo.Tyolo. Rapport d'enquête en pays sénoufo, Centre O.R.S.T.O.M. de Petit-Bassam, Abidjan, 1973, 57 p.

JAMIN J., Confacts et antagonismes culturels en pays kikuyu (Kenya), Institut d'ethnologie, musée de l'Homme, Archives et documents, Paris, 1973, 225 p.

JaMnN J., La Tenderie asux grives cbez les Ardennais du plateats. Essaj d'etbrobiologie, Institut d'ethnologie, musée de l'Homme, Archives et documents, Paris, 1974, 221 p.

JAMIN J., - De la grive imaginée à la grive imaginaire ?, L'Homme of l'Animal, Institut international d'ethnoscience, C.N.R.S., $\Lambda$ ctes du $1^{\text {er }}$ colloque d'ethnozoologie, Paris, 1975, p. 297-315. 
JAULIN R., La Mort Sara. L'ordre de la vie ou la pensée de la mort au Tchad, U.G.E., 10/18, Paris, 1971, 447 p.

Kenyatta J., Au pied du mont Kenya, Maspero, Paris, 1967, 207 p.

Krader I., Formation of the State, Prentice Hall, New York, 1968, 115 p.

LEIRIS M., La Langue secrète des Dogons de Sanga (Soudan frangais), Institut d'ethnologie, Travaux et Mémoires, Paris, 1948, 530 p.

LEscount $\mathrm{J}$, « $\mathrm{L}_{2}$ Tenderie aux grives $\gg$, Etudes ardennaises, $\mathrm{n}^{\circ} 32$, Mézières, 1963 , p. 9-14.

MARIE A., \& Parenté, échange matrimonial et réciprocité. Essai d'interprétation à partir de la société dan et de quelques autres sociétés de Côte-d'Ivoire \$, L'Homme, 1972, XII (3), p. 5-46, et XII (4), p. 5-36.

MIdDleton J., Les Kikouyous et les Kambas du Kenya. Etude scientifique sur les Mau-Mau, Payot, Paris, 1954, 158 p.

Moscovica S., La Société contre nature, U.G.E., 10/18, Paris, 1972, 444 p.

Mühlmann W.E., Messianismes révolutionnaires du tiers monde, Gallimard, Bibliothèque des sciences humaines, Paris, 1968, 389 p.

Paulme D., éd., Classes et associations d'âge en Afrique de l'Ouest, Plon, Paris, 1971, 348 p.

PERroIs L., La Circoncision bakota (Gabon), Cahiers O.R.S.T.O.M., Sciences humaines, vol. $\mathrm{V}, \mathrm{n}^{\circ} 1,1968,109 \mathrm{p}$.

Prins A., East African Age-Class Systems. An Inquiry into the Social Order of Galka, Kipsigis, and Kikuyu, Lounz, Londres, 1953, 195 p.

Prouteaux M., \& Premier coup d'œil sur la religion séné », Bulletin du Comité d'études bistoriques et scientifiques de l'A.O.F., t. IV, 1921, p. 225-251.

RABaIn-Zemplén J., Expression de l'agressivité et processus de médiation dans la socialisation de l'enfant wolof (Sénégal) $\gg$, Africa, vol. XIIV, $\mathrm{n}^{\circ}$ 2, Londres, 1974, p. 151-162.

RABAIN-ZEMPLENI J., L'Enfant wolof de deux à cinq ans, thèse de doctorat de $3^{\circ}$ cycle, E.P.H.E., VI section, Paris, 1975, 432 p.

ROGISSART J., « Des grives et des hommes », L'Atdennais, 27 novembre 1952.

Rogissart J., Passantes d'octobre, Arthème Fayard, Paris, 1958, 221 p.

ROQUePlo P., Le Partage du savoir. Science, culture, vulgarisation, Le Seuil, Science ouverte, Paris, 1974, 255 p.

Roussel L., Région de Korbogo. Etude de développement socio-économique, rapport sociologique, S.E.D.E.S., fasc. $\mathrm{n}^{\circ}$ 2, Paris, 1965, 101 p.

RourledgB W. S. et K., Witb a Prebistoric People. The Akikuyu of British East Africa, E. Arnold, Londres, 1910, 392 p.

SEJourNET, \& La Grive dans les Ardennes. Tenderie aux grives et grivières >, Almanach Matot-Braine, 1904, 46 année, p. 109-116.

SFEz L., Critique de la décision, Armand Colin, Cahiers de la Fondation nationale des sciences politiques, Paris, 1973, 367 p.

Sperber D., Le Symbolisme en général, Hermann, Savoir, Paris, 1974, $163 \mathrm{p}$.

TANTURIBR C., \& Le Gibier et la Médecine populaire \$, Etudes ardennaises, $n^{\circ}$ 32, Mézières, 1963, p. 40-42.

ZahaN D., Religion, spiritualité ot pensée africaines, Payot, Paris, 1970, $244 \mathrm{p}$. 


\section{TABLE}

INTRODUCTION $\ldots \ldots \ldots \ldots \ldots \ldots \ldots \ldots \ldots \ldots \ldots \ldots \ldots$

1. Secret cynégétique et pouvoir communal I. Le piège de la parole $\ldots \ldots \ldots \ldots \ldots \ldots \ldots \ldots \ldots, 17$

II. Le pouvoir silencieux . . . . . . . . . . . . . . 29

2. La prise de la parole dans la société lignagère

I. Le soupçon et l'accusation ............... 44

II. Pouvoir de la parole et parole du pouvoir ....... 59

3. Secret initiatique et pouvoir lignager

I. Le silence des structures ................ 65

II. Le silence des savoirs $\ldots \ldots \ldots \ldots \ldots \ldots \ldots \ldots . \quad 90$

III. De la parole retenue à la parole tenue ........ 106

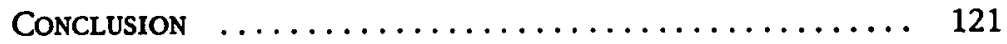

Bibliographie $\ldots \ldots \ldots \ldots \ldots \ldots \ldots \ldots \ldots \ldots \ldots \ldots, 128$ 


\title{
DOSSIERS AFRICAINS \\ dirigés par Marc Augé et Jean Copans
}

\author{
DéJর PUBlís
}

La Construction du monde. Religion, représentations, idéologies, sous la direction de Marc Augé, 1974.

Critiques et politiques de l'antbropologie, par Jean Copans, 1974.

Les Domaines de la parenté. Filiation, alliance, résidence, sous la direction de Marc Augé, 1975.

Sécberesses et famines du Sabel. I. Ecologie, dénutrition, assistance, sous la direction de Jean Copans, 1975.

Sécheresses et famines du Sabel. II. Paysans et nomades, sous la direction de Jean Copans, 1975.

L'Antbropologie économique. Courants et problèmes, sous la direction de François Pouillon, 1976.

Les Migrations africaines. Réseaux et processus migratoires, sous la direction de Jean-Loup Amselle, 1976.

Pouvoir des vivants, langage des morts. Idéo-logiques sakalava, par J.-F. Baré, 1977.

Les Lois du silence. Essai sur la fonction sociale du secret, par Jean Jamin, 1977.

\section{a paraitre}

Lo Tourisme en Afrique de l'Oust : panacée ou nouvelle traite? 


\section{PUBLICATIONS \\ DU CENTRE D'ÉTUDES \\ AFRICAINES-CARDAN}

\section{Dossiers africains}

Chaque volume de cette collection vise à faire le point de façon succincte et précise sur un domaine, un thème ou un problème concernant le continent africain. Il s'agit de mettre sous une forme accessible (aussi bien par la taille que par le contenu). une information, une réflexion et une documentation qui restent trop souvent d'accès difficile pour le profane et qui sont pourtant nécessaires à la compréhension de la société africaine contemporaine. Entre la thèse et l'article de journalisme, entre la bibliographie spécialisée et la vulgarisation touristique, il y a place pour une documentation active qui puisse aider ou guider le chercheur, l'enseignant, l'étudiant, le cadre ou le militant politique.

Ces dossiers se veulent des outils de travail, et leur présentation générale articule une synthèse originale, une bibliographie sélectionnée et commentée, des explications de textes, ainsi que des articles inédits ou difficilement accessibles. Ces dossiers se veulent directement complémentaires, ce qui fait que certains thèmes ou analyses renverront d'un dossier à l'autre. Cette série de synthèse documentaire sera complétée par des recueils d'articles ou des textes originaux qui permettront aux tendances nouvelles de la recherche en sciences sociales de s'exprimer. C'est pourquoi cette collection essaiera de signaler à l'attention de ceux qui travaillent sur l'Afrique et en Afrique les idées ou les méthodes parfois élaborées à partir d'autres terrains ou préoccupations. Cette intention est d'ailleurs un souci permanent de la collection dans la mesure où, centrée sur l'Afrique et consacrée à l'Afrique, elle démontrera néanmoins l'impossibilité d'une réflexion uniquement africaniste. Elle proposera des directions d'analyse sur les problèmes encore mal perçus ou reconnus,

- Centre d'soalyse et de recherche documentaires pour l'Afrique noire. 
qu'ils soient imposés par l'actualité sociale ou les problématiques scientifiques. Elle suggérera une reconsidération des domaines traditionnels de l'africanisme et des théories qui lui sont liées.

\section{Cabiers d'études africaines}

Cette revue paraît trimestriellement depuis 1960 . Un éventail de chercheurs de toutes nationalités, appartenant à toutes les disciplines des sciences humaines, présentent, en français ou en anglais, des études scientifiques inédites sur les sociétés, les économies, les cultures et les civilisations du continent africain. Elle comprend en outre une chronique bibliographique assurée par le Centre.

Les Cabiers d'études africaines sont publiés avec le concours du C.N.R.S. Numéros spéciaux :

$\mathrm{N}^{\circ} 30$ :

$N^{\circ} 35:$

Q Littérature orale et folklore africains $\gg$ (édité par Denise Paulme).

- Les relations de dépendance personnelle en Afrique noire • (édité par Georges Balandier).

$N^{\circ} 45:$

- Recherches en littérature orale africaine (édité par Denise Paulme).

$N^{\circ} 47$ :

$N^{\circ} 51:$

- Systèmes agraires africains \édité par Gilles Sautter).

$N^{\circ} 53:$

4 Villes africaines \(édité par Paul Mercier et Gilles Sautter).

- Problèmes de la domination étatique au Rwanda : histoire et $\mathrm{N}^{\circ} 60$ : économie (édité par Claudine Vidal).

« Thématique et symbolique des contes africains D (édité par $N^{\circ}$ 61-62 Denise Paulme).

\& Histoire africaine : constatations, contestations \& (édité par Henri Brunschwig, Catherine Coquery-Vidrovitch, Henri Moniot).

\section{Bulletin d'information et de liaison}

La collection, créée en 1969, comprend plusieurs séries, à parution annuelle (ou occasionnelle).

Inventaire de thèses et mémoires africanistes de langue franfaise soutenus : 6 fascicules parus, signalant 4019 titres.

Inventaire de thèses africanistes de langue frangaise en cours : 6 fascicules parus, signalant 4495 titres; cette série devra être absorbée par la série suivante, dont thématiquement elle fait partie.

Registre de recberches africanistes en cours : 4 fascicules parus, décrivant 1730 thèmes de recherche dans leur cadre institutionnel; les notices analytiques indiquent un certain nombre de paramètres propres à chaque recherche - auteurs, dates, financement, enquêtes et missions, méthodes, finalité de la recherche, matériaux bruts récoltés et lieux de dépôts, données bibliographiques.

Etudes africaines : inventaire des enseignements dispensés dans les pays francophones : un numéro spécial portant sur l'année 1971-1972. Organismes 
d'enseignement, programmes et enseignants, présentés dans 440 notices descriptives.

Inventaire des ressources documentaires africaines : deux numéros réalisés, portant respectivement sur les bibliothèques et centres de documentation africanistes à Paris (129 notices) et les bibliothèques de l'Afrique centrale (272 notices).

Bibliograpbie franfaise sur l'Afrique au sud du Sabara : 7 fascicules parus, totalisant 6953 références. Cette bibliographie est une réalisation conjointe des membres du Comité interbibliothèques pour la documentation africaine (Bibliothèque nationale, Fondation nationale des sciences politiques, Cardan).

Toutes ces séries, la bibliographie mise à part, traitent du continent africain (Afrique du Nord et Madagascar compris). Les informations recueillies proviennent principalement de la France, puis de tous les autres pays partiellement de langue française dans le monde. Elles sont réunies par voie d'enquêtes.

Les données sont présentées sur fiches; leur découpage et classement suivant les rubriques géographiques et/ou matières proposées permettent la constitution de ficbiers adaptés aux besoins des uns et des autres.

\section{Fiches d'ouvrages}

Cette bibliographie, signalétique et annuelle, présentée sous forme de fiches, constitue essentiellement un complément à la série bibliographique analytique, publiée par le Cardan sous le titre « Fiches analytiques » jusqu'en 1969 et, à partir de 1970, dans la « Bibliographie ethnographique de l'Afrique sud-saharienne $\triangleright$ (voir plus bas).

Les huit volumes parus de 1965 à 1975 contiennent 12840 références. A partir du volume VIII, cette bibliographie est publiée en trois fascicules, non brochés. Les notices sont classées suivant les grandes zones géographiques, subdivisées par pays. Chaque fascicule est complété par trois index : noms d'auteurs, ethnique et linguistique, systématique. L'index géographique cumulatif paraît à la fin du $3^{\circ}$ fascicule.

\section{Collaborations extérieures et autres publications}

Bibliograpbie etbnograpbique de l'Afrique sud-sabarienne : bibliographie analytique publiée annuellement par le Musée royal de l'Afrique centrale (Tervuren, Belgique), avec le concours du Cardan.

Nomenclature des populations, langues et dialectes d'Afrique noire : essai d'un inventaire descriptif, par pays, où l'on s'efforce de rassembler et éventuellement de comparer des informations relatives aux populations et aux langues (noms et synonymes, classifications et apparentements). Déjà publié : 4 Essai de nomenclature des populations, langues et dialectes de Côte-d'Ivoire » (1975, 2 vol. : 2300 fiches environ). A paraître : « Essai de nomenclature des populations, langues et dialectes du Dahomey $\gg$.

Pour tous renseignements concernant ces publications, on peut s'adresser au Centre d'études africaines, 54, bd Raspail, 75006 Paris. 
ACHEVÉ D'IMPRIMER EN AVRIL 1977 SUR LES PRESSES DE L'IMPRIMERIE AUBIN 86 - LIGUG 6 / VIENNE

DÉPôT LÉGAL : $2^{\circ}$ TRIMESTRE 1977

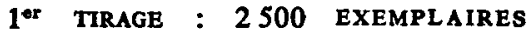

ISBN 2-7071-0920-7

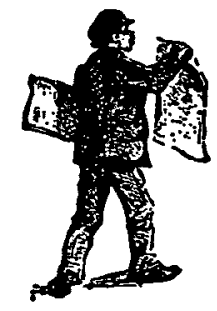




\section{dossiers africains}

dirigés par

Marc Augé et Jean Copans

La façon dont les secrets chahutent les règles de la communication, dans les sociétés traditionnelles africaines, permet d'avancer que l'oralité - qui les caractérise en partie — n'est pas forcément source et garantie d'égalité. Tout comme l'écriture, la parole se prête à des accumulations, à des confiscations, à des dissimulations et à des suspensions. Elle rentre dans des stratégies de domination et des exercices de pouvoir. Elle se distribue hiérarchiquement en raison inverse de son utilisation : c'est dire, en somme, que tout gain de pouvoir se traduit par un gain de silence, par la mise en auvre de mécanismes de pondération, de temporisation et de rétention de la parole et des discours.

La réalité sociale et culturelle des sociétés lignagères n'a pas cette transparence que lui confèrent les discours ou les théories locales, qui paraissent fonctionner sur le mode du singulierpluriel : on parle de quelques-uns pour tous, on parle à ceux dont on ne parle pas; ou, selon une loi du silence : discours qui ne disent ce quilis disent que d'une manière tendant à prouver qu'ils ne le disent pas. Que ces discours occultent ou masquent les contradictions, les rapports de force et de domination, cela ne fait aucun doute, mais ils en sont également, par ce qu'ils taisent et du fait même qu'ils le taisent, la description.

En s'interrogeant sur le statut de la parole, sur les conditions sociales de production et de reproduction des discours, sur les modes de légitimation et de transmission des savoirs - cela à partir d'études de cas qui enrayent les processus de communication sociale (secrets cynégétiques, secrets initiatiques, prestations de serment) - cet ouvrage se propose de jeter les bases d une sociologie du non-dit, ou plutôt du non-dire, et de dégager les principes d'articulation entre structures de codification, de communication et de subordination.

françois maspero, 1, place paul-painlevé, 75005 paris 